BNL-113314-2016-JA

\title{
Rapid Ordering of Block Copolymer Thin Films
}

\author{
Pawel W. Majewski, Kevin G. Yager, \\ Submitted to Journal of Physics: Condensed Matter
}

August 2016

Center for Functional Nanomaterials

Brookhaven National Laboratory

\author{
U.S. Department of Energy \\ USDOE Office of Science (SC), \\ Basic Energy Sciences (SC-22)
}




\section{DISCLAIMER}

This report was prepared as an account of work sponsored by an agency of the United States Government. Neither the United States Government nor any agency thereof, nor any of their employees, nor any of their contractors, subcontractors, or their employees, makes any warranty, express or implied, or assumes any legal liability or responsibility for the accuracy, completeness, or any third party's use or the results of such use of any information, apparatus, product, or process disclosed, or represents that its use would not infringe privately owned rights. Reference herein to any specific commercial product, process, or service by trade name, trademark, manufacturer, or otherwise, does not necessarily constitute or imply its endorsement, recommendation, or favoring by the United States Government or any agency thereof or its contractors or subcontractors. The views and opinions of authors expressed herein do not necessarily state or reflect those of the United States Government or any agency thereof. 


\title{
Rapid Ordering of Block Copolymer Thin Films
}

\author{
Pawel W. Majewski, ${ }^{1,2}$ Kevin G. Yager ${ }^{1} *$ \\ ${ }^{1}$ Center for Functional Nanomaterials, Brookhaven National Laboratory, Upton, NY, USA \\ ${ }^{2}$ Dept. of Chemistry, University of Warsaw, Warsaw, Poland \\ email: kyager@bnl.gov
}

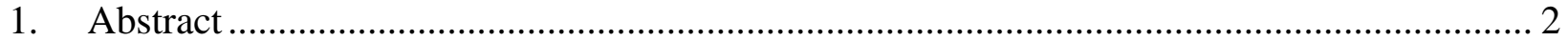

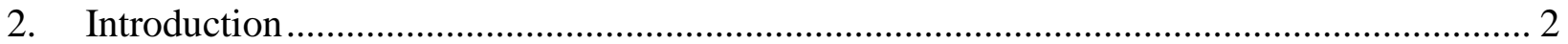

3. Fundamentals of Block Copolymer Thermal Ordering ................................................. 4

4. Characterizing Order in Block Copolymer Thin Films ............................................... 8

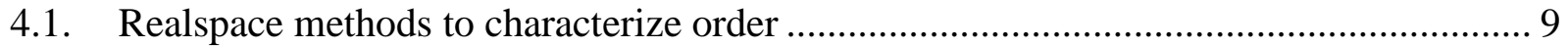

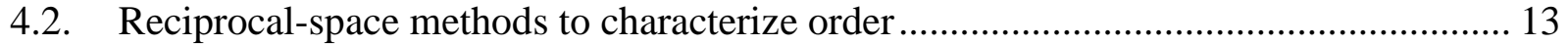

5. Ordering Using Plasticizing Additives ............................................................... 18

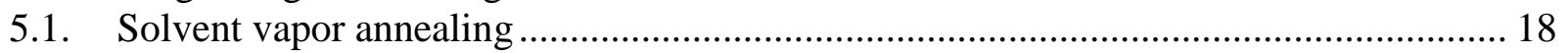

5.2. Plasticization during film casting ................................................................... 19

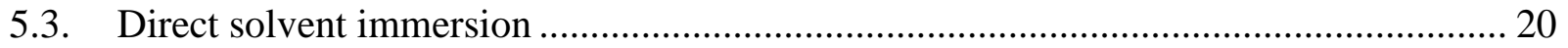

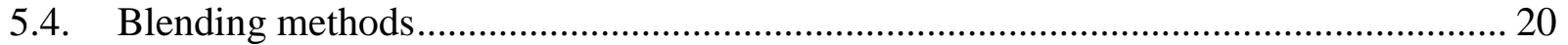



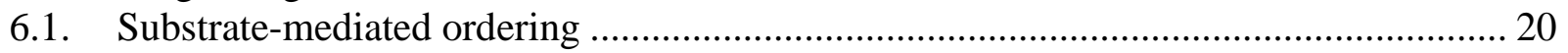

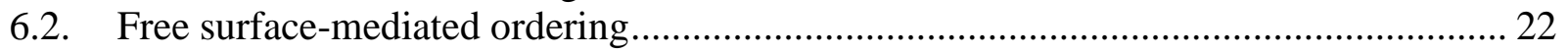

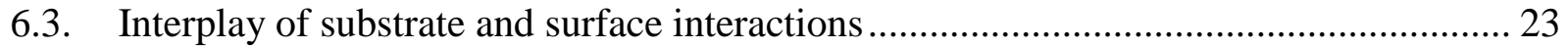

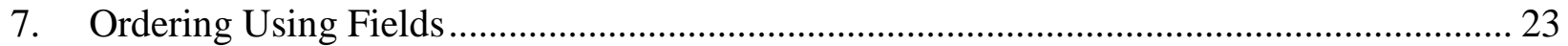

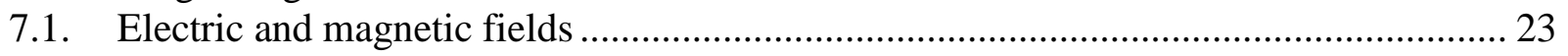



8. Ordering Using Advanced Thermal Methods ............................................................... 25

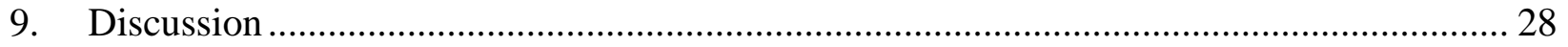




\section{Abstract}

Block-copolymers self-assemble into diverse morphologies, where nanoscale order can be finely tuned via block architecture and processing conditions. However, the ultimate usage of these materials in real-world applications may be hampered by the extremely long thermal annealing times - hours or days - required to achieve good order. Here, we provide an overview of the fundamentals of block-copolymer self-assembly kinetics, and review the techniques that have been demonstrated to influence, and enhance, these ordering kinetics. We discuss the inherent tradeoffs between oven annealing, solvent annealing, microwave annealing, zone annealing, and other directed self-assembly methods; including an assessment of spatial and temporal characteristics. We also review both real-space and reciprocal-space analysis techniques for quantifying order in these systems.

\section{Introduction}

Self-assembly is a conceptually beautiful paradigm for constructing nanomaterials. The desired structure is encoded within the architecture of the molecules themselves. Pattern formation is spontaneous, driven by energy-minimization, with molecules organizing according to intermolecular and intra-molecular association [1]. Because self-assembly is a stochastic process, assembly kinetics are of paramount importance. Although equilibrium thermodynamics in principle dictates the preferred structures, the actual order obtained may depend on nonequilibrium effects, local fields, kinetic traps, and pathway-dependent ordering [2]. Pragmatically, ordering timescales also influence the extent to which self-assembly can viably be scaled to widespread applications. This review thus delves into the ordering kinetics of selfassembling block-copolymer materials.

Block-copolymers (BCPs) represent a versatile and well-studied self-assembly motif [3-7]. Chemically distinct polymer segments are covalently connected. Chemically incompatible polymer chains experience a strong driving force towards macrophase separation [8]. However, the covalent attachment of the copolymer architecture frustrates this tendency. The compromise is for the chemically-distinct chain segments to segregate locally- "microphase separation"which results in the formation of nanoscale morphologies that minimize interfacial area, and chain stretching [3]. Linear diblock-copolymer chains segregate into morphologies dictated by the relative volume fraction of the two blocks (Figure 1a) [9-11]. Symmetric diblock chains give rise to alternating lamellae morphologies. Slightly asymmetric chains give rise to cylinders (of the minority phase) arranged in a hexagonal lattice (within a matrix of the majority phase). Greater asymmetry gives rise to spheres arranged in a body-centered cubic lattice. The diblockcopolymer phase diagram can also include regions with more exotic morphologies, such as gyroid or perforated lamellae [3, 9, 12]. More complex polymer architectures, including triblock [4, 13-20], multi-block [21-23], and bottle-brush [24-27] copolymers, can give rise to yet more elaborate morphologies [11, 28, 29]. Chain stiffness also plays a role in assembly; rod-coil and rod-rod diblock systems can be generated using rigid (possibly semiconducting) polymer blocks [30-35]. The assembly of block-copolymers can be strongly influenced by local confinement [18, 
20, 36-44]. Most simply, thin films of BCPs can exhibit new morphologies, and interfacedirecting ordering $[5,6,15,45-47]$. As will be discussed at length in this review article, the order of BCP materials can in fact be influenced in a number of different ways [48-50]. BCPs are responsive to temperature [51-53] and solvent [54, 55] conditions; to thermal [56, 57], shear [5863], electric [64-67], and magnetic [68-72] fields; and to substrate topography [73, 74] and chemical makeup [75]. One can further broaden the range of possible structures by considering blends of BCPs with other materials: especially nanoparticles [76-81], homopolymers [82-86], and other BCPs [87-96].

This elaborate landscape of morphologies has made BCPs extremely attractive as functional materials [97-99], and as a nanoscale patterning technology [23, 46, 100-104]. Furthermore, one of the key advantages of BCP self-assembly is the wide variety of established methods for converting the organic morphology into other materials [105-107]. Fixed organic structures can be obtained using various chemical treatments, including crosslinking and selective dissolution [105, 108, 109]. Metal replicas of one of the phases can be obtained via etching [39, 110-112], nanoparticle infiltration [113,114], or solution-phase exposure to metal salts [115-119]. Pyrolysis can be used to generate replicas composed of amorphous carbon [114, 120-123], silica [124, 125], vanadium oxide [126], etc. [127, 128]. Selective gas-phase infiltration can load one of the blocks with a precursor, allowing the formation of various oxides $\left(\mathrm{ZnO}, \mathrm{TiO}_{2}, \mathrm{Al}_{2} \mathrm{O}_{3}\right.$, etc.) [129-134]. These inorganic replicas can be used as lithographic etch-masks, thereby transferring the morphology into substrate topography $\left(\mathrm{Si}_{1}, \mathrm{Si}_{3} \mathrm{~N}_{4}, \mathrm{SiO}_{2}\right.$, etc.) $[40,46,135-142]$. This structural and compositional control has led to the suggestion and demonstration of a diverse array of applications $[48,49]$. One of the BCP phases can be removed to generate nanoporous materials [109], which may be useful as membranes [143], or as scaffolds for synthesizing nanowires [144]. BCP-derived nanomaterials have shown promise for energy applications [145, 146], including as battery electrolytes [29, 147-153] and electrodes [154, 155], capacitors [156], solar cell materials [35, 157-160], and triboelectric generators [161]. The ability to pattern surfaces at the nanoscale [101] has been shown to be useful for generating superhydrophobic surfaces [162, 163], gas [138] or molecular [164] sensors, optical materials [112, 118], and transparent electrodes [119]. More broadly, the ability to generated nano-patterned coatings should have an impact in light-management [165, 166] layers for anti-reflection [167], improved solar cell light harvesting [168-171], and improved light-emission efficiency [172]. BCPs have been extensively investigated as a route towards higher-resolution patterning for electronics and nano-devices [23, 145, 146, 173-175]. While a BCP locally forms a well-defined nanostructure, its position and registry at larger scales can be directed $[50,73-75,102]$ using topographic or chemical patterns; so-called directed self-assembly (DSA) patterning [176, 177]. It has been shown that DSA patterns can achieve density multiplication with respect to the guiding pattern [178-182], and that templates can enforce complex local patterns [183, 184] using minimal design rules $[180,185]$. Progressively more complex integration of BCPs into realistic industrial microelectronic workflows has been demonstrated [39, 183, 186-191]. BCPs may thus play a role in future electronics and memory devices (resistive [192, 193], phase-change [194, 195], or magnetic bit-patterned media $[137,196,197])$. As the complexity of possible patterns increases (Figure 1b-e) [39, 119, 180, 183-185, 198-202], the range of potential applications correspondingly broadens. 
In order for BCP materials to fully realize their enormous potential, a variety of outstanding challenges must be addressed [57, 203-205]. Firstly, the local nanoscale morphology must be controlled. The local orientation can be tuned using interfacial energy [75], film thickness, and thermal or solvent conditions. The local nanoscale shape is more difficult to influence [119, 141], since the morphology emerges spontaneously due to energy-minimization. On the other hand, the morphology can be influenced by confinement [44], or non-native lattice symmetries can be constructed by stacking multiple self-assembled layers [119, 141, 206-210]. This review will touch on many of these methods of local control. Secondly, self-assembly does not generically afford long-range control of the orientation or registry of the nano-objects. We note that for many applications, only relatively local (mesoscale) order matters. For instance, the optical, electrical and wettability properties of coatings is influenced by the local shape, orientation, order, and perfection of nanostructures, but not influenced by long-range order. On the other hand, anisotropic material properties require wide-area (macroscopic) control of nanoscale orientation, and device applications in microelectronics require stringent control of long-range registry. This review will discuss and distinguish the ordering in both of these regimes: mesoscale, and macroscopic. Finally, the timescales of self-assembly are frequently unacceptably long. For instance, microelectronic workflows involve numerous steps, where a timescale of only seconds or minutes can be tolerated for any given step. By comparison, conventional ordering of BCPs requires hours or days of oven annealing. This review article summarizes the state of the art with respect to block-copolymer ordering kinetics, describing both the current best fundamental understanding of the ordering process, as well as pointing towards promising techniques for accelerating BCP self-assembly. Though several challenges remain, a range of options now exist to enable rapid and efficient ordering of block-copolymers (Figure 2).

\section{Fundamentals of Block Copolymer Thermal Ordering}

Block-copolymer thin films have traditionally been ordered by heating them in a vacuum oven for extended periods (hours to days). BCPs must be heated above the glass-transition temperature $\left(T_{g}\right)$ of both block components, so that the material has sufficient mobility for the morphology to rearrange. Thermal annealing of an as-cast (disordered) BCP film involves first a rapid and local phase-separation of the BCP components, generating a disordered BCP morphology [211]. The BCP morphology then coarsens as thermal annealing proceeds, with the average grain size increasing with time (Figure 3 ). The coarsening process is dominated by the diffusion and annihilation of topological defects [212-215]. The defects in the morphology (dislocations, disclinations, etc.) $[48,216]$ behave as quasi-particles that diffuse throughout the material [217, 218]. This diffusion requires chain mobility (for the morphology to locally rearrange), but does not require long-range migration of the chains themselves. As coarsening proceeds, the density of defects continuously decreases, reducing the probability for two defects to encounter and annihilate. Coarsening leads to an increase in grain size $(\xi)$ that scales as $\xi=k t^{\alpha}$, where $t$ is time (Figure 3d). The prefactor $k$ sets the overall scale of the kinetics, including the effect of temperature, while the exponent $\alpha$ describes the mechanism of ordering. For thin films, ordering is quasi two-dimensional (2D), and one expects $\alpha=0.25$, with experimental values being in the 
range $[215,216,219-223] \alpha \approx 0.25-0.28$ for sphere and cylinder morphologies, and closer to 0.1 for lamellae [213, 222] (Figure 4). By comparison, bulk materials have three-dimensional (3D) freedom, and exhibit $[212,224] \alpha \approx 0.5-1$.

The topological defects are deviations from the idealized morphology (Figure 3a and b, example defects are highlighted by red circles), and thus inherently high-energy, requiring the underlying BCP chains to distort unfavorably. Elimination of defects is thus an energetically downhill process. Nevertheless, grain coarsening is limited by both the finite diffusivity of the defects, and an energy barrier for two defects to annihilate (since annihilation requires higher-energy intermediate configurations [225-227]). Fredrickson et al. quantified the thermodynamic and kinetic aspects of defects using simulations [225, 226], finding that each defect incurs an energy penalty of order 10-150 $k_{B} T$, and elimination of a single defect pair involves a pathway with 1-4 energy barriers, of height $\sim 1-20 k_{B} T$, giving rise to the observed activation energy $\left(E_{a}\right)$. Increasing temperature $(T)$ increases the diffusion velocity of defects, and also provides the necessary energy to overcome the barrier to annihilation; this dependence can be captured in an Arrhenius relation [213, 217, 228-230]:

$$
k=A e^{-E_{a} / k_{B} T}
$$

where $k_{B}$ is the Boltzmann constant, $E_{a}$ is the activation energy, and $A$ is a prefactor accounting for other aspects of ordering. The prefactor accounts for the overall scale of the kinetics, including factors such as the mobility and collision frequency of defects. This parameter is thus influenced by material properties such as the diffusivity of BCP chains. For instance, high molecular-weight BCP materials have slow chain diffusion, and correspondingly slow ordering kinetics (smaller $A$ ). While the ordering exponent $(\alpha)$ allows one to assess the mechanism of ordering, and thus to qualitatively compare the ordering process inherent to different annealing protocols, the prefactor $(A)$ sets the overall scale of the kinetics, and is thus crucial in assessing the viability of a given ordering protocol in, e.g., an industrial setting. Different ordering methods influence not just the exponent, but also the prefactor (Figure 4). Thus, kinetic enhancements can arise both from accessing new ordering mechanisms, as well as by accelerating known pathways.

It is important to note that the temperature-dependence of BCP coarsening is not strictly Arrhenius, due to the multiple energy barriers [225, 226] and temperature-dependent material properties [228, 231, 232]. Moreover, as $T$ is increased, the BCP approaches the order-disorder transition (ODT). That is, the segregation strength $(\chi)$ is temperature-dependent (Figure 5a), $T \sim$ $1 /(\chi N)$, where $N$ is chain length [233-236]. Nevertheless, BCP ordering is frequently analyzed as an Arrhenius process, where the activation energy is found to be on the order of $[213,217,228$, 229, 237, 238] 50-400 kJ/mol.

Given the favorable scaling of kinetics with annealing temperature, an obvious approach to rapid assembly is simply to increase temperature. However, one is inherently limited by the orderdisorder transition temperature $\left(T_{\mathrm{ODT}}\right)$, above which phase separation no longer occurs and the two block materials mix freely (forming a disordered state). In practice, material degradation temperatures may also be limiting. For instance, polystyrene degrades at [239] $\sim 380^{\circ} \mathrm{C}$, 
polyethylene $\sim 420^{\circ} \mathrm{C}$, poly(methyl methacrylate) [240] $330^{\circ} \mathrm{C}$, polyvinylpyridine [241] $\sim 400^{\circ} \mathrm{C}$, poly (ethylene oxide) $[242,243] \sim 400^{\circ} \mathrm{C}$, poly (dimethyl siloxane) $[244] \sim 500^{\circ} \mathrm{C}$, etc. In most cases, researchers limit themselves to $T<250^{\circ} \mathrm{C}$ to avoid possible degradation issues. Rapid thermal processing (RTP) was used to investigate high temperatures (up to $350^{\circ} \mathrm{C}$ ) [237, $245,246]$, where it was found that initial ordering was enhanced $(\alpha \approx 0.3)$, quickly plateauing to the usual behavior $(\alpha \approx 0.1)$. Overall, this enables significant grain sizes within $\sim 100 \mathrm{~s}$.

Ultimately, ordering of a BCP morphology results from the rearrangement of the underlying polymer chains. Thus, the microscopic molecular properties (e.g. polymer relaxation times) play a crucial role in dictating ordering kinetics (Figure 5). Conversely, the morphology influences molecular properties, such as the diffusion of block-copolymer chains. For instance, in lamellar systems, chains diffuse readily within the lamellar plane, but there is an energy barrier for chains to diffuse in the perpendicular direction (i.e. to hop between layers), giving rise to diffusion coefficients approximately one order-of-magnitude lower in this direction [222, 247-249].

Spin-cast BCP films are typically initially disordered; but this state is meta-stable, since the material is well below $T_{\mathrm{ODT}}$, and will rapidly phase separate as soon as mobility is induced (by heating above $T_{g}$ ). Heating disordered films results in homogeneous phase separation throughout the entire material, with subsequent grain growth involving some grains growing at the expense of others (Figure 3a,b). Alternatively, BCPs can be ordered by heating the material above $T_{\text {ODT }}$ (into the disordered phase), and cooling through the order-disorder transition. In this case, one instead observes nucleation and growth of isolated ordered grains (in a surrounding of disordered material). These grains typically grow anisotropically (yielding grains with ellipticity 2) [250253], with front velocities on the order of $10 \mu \mathrm{m} / \mathrm{min}$. The kinetics of grain growth within this supercooled regime was studied in detail by Fredrickson and Binder [254]. The energy barrier for nucleation of ordered grains is quite small and decreases with quench depth; for substantial quenches, rapid homogeneous nucleation is inevitable (i.e. growth of individual, isolated grains occurs only in the vicinity of $\left.T_{\mathrm{ODT}}\right)$. The timescale for conversion to an ordered state follows:

$$
t_{\text {ord }} \sim \frac{N^{1 / 12}}{\delta^{3 / 4}} \tau_{d} e^{\Delta F / 4 k_{B} T}
$$

where $\tau_{d}$ is the polymer relaxation time, $\delta$ is proportional to the undercooling, and $\Delta F$ is the excess free energy of formation of a droplet of ordered material.

Conventional thermal annealing can only, realistically, ever offer mesoscale ordering. The kinetics of assembly are too slow for macroscopic grain sizes to be obtained over reasonable timescales. If macroscopic order is desired, some other method must be used to select a preferred orientation, or template large grains. In this review, several techniques which afford this level of control are discussed. From the point-of-view of conventional annealing, these techniques represent an enormous kinetic speedup $\left(\sim 10^{20}\right)$.

The intrinsic sizescale of a BCP morphology leads to interesting commensurability effects. For instance, a BCP material confined between two hard walls will form an integer number of morphological repeats within the available space, at the expense of distorting $L_{0}$ from its bulk equilibrium value [255]. Yet this distortion is manifestly high-energy; if a BCP thin film is cast 
on a substrate with a free surface, one will instead see a smooth flat surface when the film thickness admits an integer number of BCP repeats. But if the average film thickness is not commensurate, the BCP will respond by nucleating 'holes' or 'islands' at the surface [256-258]. That is, the free surface will partition itself into regions slightly thicker and thinner than the average thickness (with the quantized thicknesses having a height difference of $L_{0}$ ), so that any given spot admits an integer number of layers. For a lamellar film under symmetric wetting conditions (the same block wets the substrate and free surface), the film is commensurate for any thickness $n L_{0}$, where $n$ is an integer. For asymmetric wetting (different block wets substrate $v s$. surface), commensurability occurs for $(n+1 / 2) L_{0}$. For sphere and cylinder morphologies, one must consider the correct layering distance, and account for any substrate-wetting brush [46]. If either the substrate or free interface is near-neutral, one can also form half-height $\left(0.5 L_{0}\right)$ surface features [259].

Thicknesses slightly above commensurability have an excess of islands on their surface; those slightly below commensurability exhibit holes; intervening thicknesses can display bicontinuous surface features [260]. These surface features nucleate as small, smooth bumps/divots, which then grow in size and exhibit relatively sharp walls [261, 262]. The appearance of these features is preceded by an incubation phase [258, 263, 264]: a meta-stable state where the film is nominally flat. The timescale for initial nucleation depends sensitively on film thickness, with near-commensurate thicknesses exhibiting long ( $>10$ hours) incubation, while 'maximally incommensurate' thicknesses (exactly between two commensurate conditions) nucleate rapidly (tens of minutes) [265]. The additional surface area of these surface features introduces an energy penalty, which causes these features to limit their perimeter, and to coalesce as annealing proceeds [266, 267]. Yet their evolution involves the organized motion of many polymer chains; nucleation and growth occur on timescales of hours [258, 267], with coarsening following a power law as a function of time [268].

Film thickness affects BCP morphologies. Thin film confinement can give rise to morphologies that deviate from the bulk equilibrium expectation [15, 45, 47, 269, 270], or induce reorientation [15, 271-274]. Local film thickness (e.g. at a step in thickness) can also induce local morphological changes [272, 275, 276]. Given this coupling between thickness and morphology, it is not surprising that film thickness also affects ordering kinetics. Generally, thicker films order more rapidly. A limiting case is to compare bulk materials, which have freedom to order in $3 \mathrm{D}$, to thin films, which are confined to quasi-2D ordering. Coarsening follows $\xi \sim t^{\alpha}$, where bulk films exhibit $[212,224] \alpha \approx 0.5-1$, while 2 D films order with a less favorable [215, 216, 219223] $\alpha \approx 0.25$. For thin films annealed at a certain temperature and time, the grain size is larger for thicker films, scaling as $\xi \sim h^{\beta}$, where $h$ is thickness, and [223, 274, 277, 278] $\beta \approx 0.4-1$. The faster ordering in thicker films can be attributed to the decreased influence of confinement and substrate effects. Increasing ordering kinetics using thickness is not generally viable: the kinetic increase is marginal, and other aspects of a particular use/application typically restrict the film thickness that is acceptable. Moreover, beyond a certain thickness, one no longer obtains grains that span the entire film thickness, increasing the effective disorder. 


\section{Characterizing Order in Block Copolymer Thin Films}

A key question, when assessing self-assembling ordering rates, is how best to quantify the order that develops in the system. The appropriate metrics depend on the intrinsic shape and symmetry ordering of the system, as well as the length-scale over which the order persists. For blockcopolymer thin films, we can distinguish between different regimes. Early in the phase separation process, or close to the order-disorder transition (ODT) boundary, order will be only extremely local. One can characterize the system by the interfacial width $(W)$ between the two block components [279-281]:

$$
W=\frac{a}{\sqrt{6 \chi}}
$$

where $a$ is the polymer statistical segment length. The interaction parameter $(\chi)$ is temperaturedependent, with an entropic $\left(\chi_{S}\right)$ and enthalpic $\left(\chi_{H}\right)$ contribution [234]:

$$
\chi(T)=\chi_{S}+\frac{\chi_{H}}{T}
$$

Figure 5a shows the variation in $\chi$ for various BCPs (PS- $b$-PMMA [233-235, 282], PS- $b$-PVP [283-285], PS- $b$-PEO [286], PS- $b$-PI [233], and PS- $b$-PDMS [287, 288]). Near ODT, the interfacial width increases as the two block components begin to mix, and diverges as one crosses ODT. As temperature is lowered, and effective segregation strength increases, the interfaces become sharper ( $W$ decreases). Spin-cast BCP films are frequently in kineticallytrapped disordered configurations, and require oven annealing (above $T_{g}$ ) to induce mobility and allow the system to evolve into an ordered state. Thus, one can use the interfacial width, $W$, as a measure of the extent of phase separation during this early-stage ordering.

This initial phase separation is typically extremely rapid. BCP ordering is thus dominated by the next regime, wherein the morphology has developed, defects diffuse and annihilate, and order thus improves. These states are 'poly-grain,' with an assortment of domains/grains of different size, separated by defective grain-boundaries. The system is well-ordered locally (at the scale of 1-20 $L_{0}$ ), but disordered globally; we can refer to this as the mesoscale ordering regime. One can thus characterize order using the areal or volumetric density of defects $\left(\rho_{d}\right)$. The most popular metric, however, is to measure the average grain size $(\xi)$; i.e. the distance over which the nanoscale morphology is coherent. In principle, the enthalpy-minimum for a BCP system is to generate a single grain throughout the entire macroscopic dimensions of the sample. In practice, such a condition requires impractically long annealing times. On the other hand, a wide variety of methods are available to direct BCP ordering. For instance, shear-alignment can break the two-dimensional powder symmetry of a thin film, giving rise to a morphology aligned along a particular direction. In this macroscale ordering regime - where long-range correlations have been established - the grain size does not provide a meaningful description of the system. Depending on how $\xi$ is measured, it may in fact diverge to infinity in such a system, since (certain) correlations persist over arbitrary distances [57, 289]. In such cases, one can instead focus on counting the density of remaining topological defects $\left(\rho_{d}\right)$; or one can measure the deviation of the system from idealized mono-domain order. For instance, the orientational spread 
of the morphology, about the average alignment direction, can be considered $\left(\sigma_{\phi}\right)$. The Hermanns order parameter $(S)$ can be computed by averaging the local angles $(\phi)$ of the morphology with respect to the 'director' (alignment direction) [278, 290-292]:

$$
S=\left\langle P_{2}(\cos \phi)\right\rangle=\frac{3\left\langle\cos ^{2} \phi\right\rangle-1}{2}
$$

where $P_{2}$ is the second Legendre polynomial. The order parameter allows a simple interpretation: a disordered system with completely random orientations yields $S=0$, a perfectly-aligned system yields $S=1$, and a perfectly anti-aligned system yields $S=-1 / 2$. Intermediate values of $S$ (between 0 and 1) allow one to sensitively characterize and compare orientational order. In twodimensions (2D) one can instead use [289]:

$$
\begin{aligned}
S & =2\left\langle\cos ^{2} \phi\right\rangle-1 \\
& =\langle\cos (2 \phi)\rangle
\end{aligned}
$$

Once a metric has been selected, the ordering kinetics are of course established by probing this metric as a function of ordering time. As previously discussed, BCP ordering typically follows a power-law, with metrics (such as grain size) improving rapidly in initial phases, and then growing progressively more slowly as defects become more dilute.

\subsection{Realspace methods to characterize order}

Conceptually, the simplest way to characterize a morphology is to directly visualize it. In later stages of ordering, incommensurate BCP thin films may form island/hole surface patterns [256258]. These can be visualized using optical microscopy,[260] and their size/shape tracked over time [267]. This allows the latest stages of assembly to be analyzed relatively easily. The nanoscale morphology of a BCP, however, is well below the wavelengths of visible light, making optical microscopy inapplicable to measuring the metrics of greatest interest $\left(\xi, \rho_{d}, S\right.$, etc.). On the other hand, Ullal et al. [293, 294] demonstrated how stimulated emission depletion microscopy (STED) could be used to image a BCP phase doped with a block-selective fluorophore, with sub-diffraction-limit resolution. Pollard et al. used infrared-based scanning near-field optical microscopy (SNOM) to yield spectroscopic imaging of a BCP with nanometer spatial resolution [295]. It is conceivable that other super-resolution microscopy [296, 297] methods could be adapted to BCP imaging.

By far the most common means of assessing BCP morphologies is realspace visualization with atomic force microscopy (AFM) or scanning electron microscopy (SEM). Both techniques allow the morphology at the top-surface of a thin film to be imaged. However, both are encumbered by a perennial issue in BCP imaging: the morphology of interest may be buried under a wettinglayer of the lower surface-energy component. AFM operating in intermittent-contact ('tapping') mode can image the subsurface morphology; this is especially evident in the phase image. For SEM imaging, a development step is usually required, such as a brief etching (e.g. with $\mathrm{O}_{2}$ plasma) to remove the surface layer and differentially consume the two block components. For some BCP materials, such as PS- $b$-PMMA, one can use UV irradiation to crosslink the PS phase, 
and acetic acid exposure to remove the PMMA phase, thereby generating height contrast visible in SEM. In any case, SEM imaging of these organic materials is usually improved by depositing a thin metal layer (e.g. $5 \mathrm{~nm} \mathrm{Pt}$ ), which reduces charging effects. As demonstrated by Hexemer $e t$ al. [298], the throughput of these imaging modes can be enhanced by using wide-area images and analyzing the Moiré patterns. The interference between the regular pattern of the BCP morphology, and the regular grid of the microscope scanlines, gives rise to a visual 'beating.' These Moiré patterns can be rigorously analyzed to determine the local BCP morphology, even though the repeat-spacing is below the nominal scan resolution. As such, orientational order and grain size can be evaluated.

AFM requires no preparation of the material for imaging, and can even allow the morphology to be imaged during ordering (e.g. using a heated sample stage), allowing coarsening kinetics at video rate to be measured [299, 300]. In this sense, AFM provides the only truly non-destructive realtime measure of BCP ordering. SEM cannot measure structure in realtime; however, kinetic history can be established from SEM data by preparing a series of samples quenched at various points in the history. The temporal resolution possible in such a case is limited not by the technique itself, but by the quench protocol (resolution better than $\sim$ seconds is challenging).

In order to extract quantitative measures of order from AFM or SEM micrographs, image analysis can be used. A variety of techniques have been described in the literature. Murphy et al. provide a detailed description and integrated implementation of a suite of useful analysis tools [289]. A fast Fourier transform (FFT) of the image yields a useful reciprocal-space view of the order. The one-dimensional azimuthally-averaged FFT exhibits a peak from the BCP repeatspacing [301]. Fitting the first peak position $\left(q_{0}\right)$ allows easy computation of realspace repeatdistance $\left(d=2 \pi / q_{0}\right)$. One must account for lattice symmetry when interpreting this value. For a lamellar phase, the BCP repeat period is simply $L_{0}=2 \pi / q_{0}$. For hexagonally-packed cylinders [302], the scattering peak directly probes the layering distance between cylinder rows $L=2 \pi / q_{0}$, with the intercylinder repeat period then being $L_{0}=(2 / \sqrt{3}) L$. The orientation distribution of the morphology can be assessed from the intensity of the FFT peak along the azimuthal direction $(\phi)$, from which one can compute the order parameter $(S)[211,291]$. For well-oriented materials, one can fit the peak along $\phi$ to a Gaussian, in order to compute the spread, $\sigma_{\phi}$. A more generalized fit to the orientation distribution is to use a function with circular wrapping, such as [303, 304]:

$$
I(\phi)=\frac{1-\eta^{2}}{(1+\eta)^{2}-4 \eta \cos ^{2} \phi}
$$

where $\eta$ is an orientation parameter reminiscent of the order parameter; $\eta=0$ corresponds to complete and uniform orientational spread, $\eta=1$ corresponds to perfect alignment, and intermediate values correspond to different amounts of alignment. Another option is to fit $I(\phi)$ to the Maier-Saupe distribution [305]:

$$
I(\phi)=\frac{1}{c} \exp \left[m \cos ^{2}\left(\phi-\phi_{0}\right)\right]
$$


where $c$ is a normalization constant, and $m$ quantifies alignment; $m=0$ indicates an isotropic distribution, while $m \rightarrow \infty$ indicates perfect alignment.

Images of BCP morphologies can be cleaned using Fourier filtering; i.e., by suppressing or accentuating certain regions of the Fourier space, before performing an inverse Fourier transform back to a realspace image. For instance, if one suppresses all the intensity in the Fourier data outside of a narrow annulus near the morphology peaks, then the corresponding realspace image will have the morphology strongly highlighted [211]. Small-scale fluctuations in the image (large- $q$ in Fourier space), as well as large-scale drift in the image (small- $q$ in Fourier space), will both be rejected in this process. This greatly improves image contrast, and makes morphological features more evident. One must, however, be careful in applying this method to noisy images: by rejecting all data outside of the $q$ presumed to correspond to the $\mathrm{BCP}$ morphology, the resulting realspace image will inevitably display structure at the corresponding realspace repeatspacing. Thus, while Fourier filtering can help to highlight existing morphology, it can mislead by generating a semblance of morphology out of noise in the input image.

The average grain size can be computed from a micrograph. One could manually identify grains and grain boundaries, compute their areas, and thereby measure an area-averaged grain size $\left(\xi_{A}\right)$. A more computationally tractable method is to compute the in-plane orientational correlation length $\left(\xi_{o}\right)$, and use this as a representation of the grain size. The first step is such an analysis is to determine the local orientation of the morphology. For line-patterns (vertical lamellae or horizontal cylinders) this can be done using FFTs in sub-regions of the image [306], by computing the tangents of 'objects' identified in the image via thresholding and/or segmentation [289], or by measuring local image gradients [56, 215, 216, 229, 278]. In this latter method, spatial derivatives are used to compute local vector orientations for every pixel in the image. For an image with intensities $I(x, y)=I(\vec{r})$, we then compute the orientation map:

$$
\phi(\vec{r})=\frac{1}{2} \arctan \left[\frac{2\left(\nabla_{x} I\right)\left(\nabla_{y} I\right)}{\left(\nabla_{x} I\right)^{2}-\left(\nabla_{y} I\right)^{2}}\right]
$$

Note that care must be taken in order for the correct quadrant to be selected in computing the arctan (many programming languages have an 'atan2' function for this purpose). This method requires either preprocessing the image to remove features in the image at higher frequencies than the morphology (via Fourier filtering, or convolving with a Gaussian) [301], or taking care to compute the spatial derivatives over a length-scale of roughly $L_{0}$. The orientation map itself is extremely valuable. If plotted as false-color, it provides a direct visualization of the grains (regions over which color is conserved), grain boundaries and defects (where color changes abruptly), and meandering of the morphology (where color changes gradually). Refer to Figure 3a,b for examples. The histogram of orientations can be used to assess order or quantify orientational spread $\left(\sigma_{\phi}\right)$. One can also compute the orientational correlation function:

$$
g(r)=\left\langle\cos \left[2\left(\phi(\vec{r})-\phi\left(\vec{r}^{\prime}\right)\right)\right]\right\rangle
$$


where $r$ is a separation distance, and the angle brackets denote averaging over all possible pixel pairs (at positions $\vec{r}$ and $\vec{r}^{\prime}$ ) that match the given separation. The correlation function decays as a function of $r$, with a length-scale characteristic of the spatial persistence, in the original image, of the local orientation of the morphology. One can quantify this by fitting $g(r)$ to an exponential decay (Figure 3c):

$$
g(r)=e^{-r / \xi_{o}}
$$

where $\xi_{o}$ is the orientational correlation length. Large values of $\xi_{o}$ indicate orientational coherence over large distances (i.e. large grains) and good order. Small values indicate small grains and poor order. Note that $\xi_{o}$ is a fairly stringent measure of order, since it includes contributions both from abrupt changes in orientation (grain boundaries), as well as more subtle meandering of the morphology. It is thus smaller than what one sees 'by eye' in the micrograph (i.e. smaller than $\xi_{A}$ ). On the other hand, for aligned systems $\xi_{o}$ can be misleading. If there is a net alignment, then the orientational correlation never decays to zero. The correlation function can still be a useful indicator of order: the decay at small $r$ encodes local meandering of the morphology, while the height of the plateau at large $r$ relates to the overall orientation spread. In general, metrics other than $\xi_{o}$ should be used for aligned systems (or in cases where grains are larger than the available image size).

For dot-patterns (vertical hexagonally-packed cylinders), the above image gradient method is not applicable. Instead, the locations of cylinder cores can be obtained by thresholding the image and using standard particle localization/counting methods. From there, one can obtain Voronoi plots [223, 307] of the hexagonal order (wherein cells that are not six-sided can be identified as defects), and estimate grain size from the decay of the autocorrelation function [308]. The fraction of cylinders with exactly six nearest-neighbors can be used as another measure of order. One can also compute the orientation of each cylinder core by establishing vectors to its nearestneighbors, and then determining the angle of the average vector (one must modulo the angle into the range $-\pi / 6$ to $+\pi / 6$ to account for the six-fold symmetry) [57]. Once an orientation map is obtained, the correlation length can be computed as before (using a value of 6 instead of 2 for symmetry).

Individual topological defects can also be identified and counted using image analysis [309]. Processed images can be segmented into objects/particles, and then skeletonized into thin lines [289]. The connectivity of this network can be analyzed to identify defects and compute defect density $\left(\rho_{d}\right)$. The skeletonized network can also be used to calculate local line-edge roughness (LER) and line-width roughness (LWR).

Some BCP thin films may exhibit a mixture of morphologies or orientations of the morphology. For instance, cylinder phases may exhibit a mixture of vertical domains (which appears as hexdot regions) and horizontal domains (which appear as lines). These can be differentiated in image analysis using thresholding and particle-counting routines. A size cutoff can then easily differentiate between the smaller dots and the larger lines; flood-fill methods can expand these objects into domains throughout the image [95]. For lamellae, the absolute magnitude of local image gradients can be used to differentiate between regions of horizontal lamellae (which 
appear featureless and thus have small gradients) and regions of vertical lamellae (which have striping that generates large gradients) [310].

Overall, image analysis provides a robust and powerful means of quantifying the ordering of BCP thin films. The main drawback is that AFM and SEM are inherently surface-sensitive techniques. It is not always clear whether the ordering observed on the surface is representative of the order through the film. Indeed examples of surface-specific phenomena in BCP ordering are known [311].

\subsection{Reciprocal-space methods to characterize order}

$\mathrm{X}$-ray and neutron scattering have emerged as powerful tools for quantifying the order in selfassembling soft matter systems. While wide-angle experiments probe atomic or molecular scale ordering and crystallization, small-angle scattering (SAS) is sensitive to nanoscale order [312317]. Ordered BCP phases give rise to distinct peaks or rings in small-angle scattering; these are diffraction peaks from the nanoscale repeating-structures, and are thus precisely analogous to the wide-angle diffraction peaks one observes for atomic crystals. SAS data is obtained by recording the intensity of scattering rays as a function of the angle with respect to the direct beam $(2 \theta)$ on a two-dimensional detector; data is then converted into 'reciprocal-space' by considering the momentum transfer vector, whose magnitude is:

$$
q=\frac{4 \pi}{\lambda} \sin \theta
$$

where $\lambda$ is the wavelength of incident radiation. Both small-angle x-ray scattering (SAXS) and small-angle neutron scattering (SANS) have been found to be valuable for studying soft matter. The former relies on weak differences in electron density to yield scattering contrast, but can take advantage of the prodigious photon flux available at modern synchrotron facilities [318]. The latter exploits nuclear scattering, and can thus greatly enhance scattering contrast by isotopic selection (especially deuteration of one of the hydrocarbon blocks), compensating for the poorer flux of neutron reactor or spallation sources. A scattering experiment roughly returns the Fourier transform of the sample's realspace structure; more strictly, experimentally the detector records the intensity (square of the amplitude) of the scattered waves. The lack of phase information makes direct inversion of this Fourier data impossible. Scattering data is inherently informationpoor in the sense that data-fitting is ill-posed (there are multiple realspace structures that will both fit a given intensity profile). Despite the fact that scattering data is not intuitive - and is potentially difficult to analyze - these techniques have many unique advantages over realspace microscopy. Atomic and nanoscale resolution are easily obtained, and most samples can be studied without staining, etching or other preparations. X-ray and neutron beams are highly penetrating, allowing bulk samples and subsurface order to be studied, as well as in-situ measurements in liquids or custom sample cells. Furthermore, while microscopy is an inherently local measure of order, scattering provides complementary data by yielding quantifications of order that are averaged over large sample volumes, and are thus statistically strong. High-flux synchrotron x-ray beams allow fast (sub-second) measurements; combined with in-situ capabilities (heating, solvent exposure, etc.), this allows the kinetics of ordering to be measured. The low flux of neutron sources makes realtime in-situ monitoring of BCP coarsening kinetics 
impractical (neutron scattering data collection requires minutes to hours). However, high-flux synchrotron x-ray beamlines are extremely well-suited to high-speed data acquisition (down to milliseconds), making realtime observations of BCP phase transitions, and ordering kinetics, readily possible.

Small-angle scattering has been applied extensively to studying block-copolymer materials [319322]. Scattering data from well-ordered BCP phases will exhibit a series of rings, whose relative position and intensity can be indexed to determine the symmetry, and thus morphology. A variety of models have been presented for quantitatively fitting scattering data of nanostructures [304, 323-328]; these models can easily be adapted to fit data from BCP materials. The intensity along a scattering ring in the azimuthal direction can be used to determine the orientation distribution, from which the previously-described metrics $\left(\sigma_{\phi}, S, \eta\right.$, etc.) can be computed. One can probe a sample at different orientations with respect to the beam, allowing the orientation in three-dimensions (3D) to be assessed. More broadly, one can rotate the sample in the beam, and use images collected at various angles to reconstruct the full 3D reciprocal-space of the sample. These orientation analyses are especially important for samples with macroscopic order. A variety of methods exist to align BCP phases; however the quality of alignment can vary drastically depending on the method and processing protocol. Scattering allows this orientation distribution to be quantified.

The width of a peak in SAXS can be generically interpreted as a measure of average correlation length: large, well-ordered grains generate intense, sharp diffraction peaks; whereas small grains lead to broad scattering peaks. This can be quantified through the Scherrer equation [329-332]:

$$
\xi_{s}=\frac{2 \pi K}{\Delta q}
$$

where $\Delta q$ is the peak width in reciprocal-space, $K$ is the Scherer constant, and $\xi_{s}$ is the correlation length (Figure 6b). The constant $K$ is of order unity, and depends on a variety of factors, including the grain shape, the symmetry of the unit cell giving rise to scattering, and the peak width definition being used (FWHM, standard deviation, etc.) [330]. The correlation length is most frequently directly interpreted as being the average crystallite size; in the case of a $\mathrm{BCP}$, this would be the grain average size (i.e. the typical distance over which the morphology is positionally and orientationally coherent). However, some caution is necessary, as a variety of effects can contribute to peak broadening in SAXS data. The most mundane contribution is that instrumental resolution must not be forgotten [332]. This also sets a limit to the maximum grain size that can be quantified using a Scherrer analysis. Strain in a material can give rise to a distribution in repeat-spacings, which will also broaden a peak [333, 334]. The grain size distribution also plays a role [335]. Note also that even materials without grain boundaries will have a finite $\xi_{s}$ because internal disorder decorrelates the structure over large enough distances. This 'paracrystal' state [336-338] can often dominate in soft-matter systems. These different sources of broadening can, in principle, be distinguished based on peak shape and variation in peak width/shape in different reflections [339, 340]. 
Care must also be taken when comparing grain sizes calculated in different ways. The Scherrer analysis computes the volume-averaged grain size. Comparisons to orientational grain sizes can be misleading, since $\mathrm{x}$-ray scattering is sensitive to both orientational and positional decorrelation. Well-aligned BCP materials (orientation correlation over infinite distances) will nevertheless have imperfect registry over long distances (finite coherence measured in scattering). In this sense, the grain size measured by scattering is a very stringent measure of order. There is an upper limit to the grain size that can be measured using a peak width analysis, since instrumental effects (beam divergence, wavelength spread, etc.) impose a minimum peak width. For a modern synchrotron beamline, this cutoff typically limits one to measuring submicron grain sizes. An alternative 'variance scattering' method has recently been proposed for measuring grain sizes [341, 342], wherein one quantifies the statistical variation of intensity along a scattering ring. A large population of small, randomly-oriented, grains will give rise to a uniform scattering ring (as the innumerable Bragg peaks overlap), whereas a smaller number of larger grains will give rise to a scattering ring with significant intensity variation (in the limit of just a few grains, one can in fact resolve the individual Bragg peaks). This method bypasses the peak width limit, and thus enables quantification of grain size across a wide range (nanometers to hundreds of microns). This method also probes a volume-averaged grain size, though there is a subtly different definition of grain (average number of distinguishable domains) compared to the Scherrer method (length-scale over which lattice is coherent).

$\mathrm{X}$-ray scattering has been adapted to study thin films. In grazing-incidence small-angle $\mathrm{x}$-ray scattering (GISAXS), a focused x-ray beam is reflected off of the sample/substrate interface (Figure 6a) [343-349]. Although x-rays are strongly penetrating, at sufficiently shallow angle (on the order of $0.1^{\circ}$ ), total external reflection of the beam is observed. In this geometry, the small $x$ ray beam is projected (along the beam direction) into a large stripe over the sample surface. As such, the total scattering volume is sufficiently large to resolve a signal from even just monolayers of nanomaterials. Moreover, reflection-mode effects (such as waveguiding [350352] of the beam within the thin film) can further enhance the signal. One can also exploit the incident angle to perform 'depth profiling' of thin films. At sufficiently shallow angle, the beam will totally reflect from the film-ambient interface, and thus only probe (evanescently) the top few nanometers of the film. At a slightly higher angle - above the film-ambient critical anglethe beam penetrates to the substrate, and the resultant scattering pattern will be representative of the structure throughout the film. One can thus compare the structure at the surface $v s$. within a film, resolving the ambiguities inherent to AFM and SEM. With some care, one can more gradually vary the incident angle, to reconstruct the structure as a function of film depth (rather than simply the average order through the film) [47, 350, 353-357]. GISAXS has thus emerged as a powerful means for studying nanostructured soft materials, including studying them in-situ during assembly or conversion; block-copolymers have been extensively studied in this way [358-365]. With appropriate corrections, GISAXS data can be used to identify unit cell [366], morphology, repeat spacing, orientation distribution (Figure 6c) [367-370], grain size (Figure 6b) [332], etc. In fact, properties can be computed anisotropically (in-plane $v s$. out-of-plane). It should be noted that for a thin film the film thickness itself represents a maximum possible 'grain size' in the vertical direction. Very thin films thus have peaks in GISAXS that are very broad 
along the film normal direction ( $q_{z}$ in reciprocal-space), appearing as streaks. More generally, one must be aware that in GISAXS the film thickness itself gives rise to scattering features.

Although GISAXS is a powerful technique, the data one obtains is even more difficult to analyze than conventional scattering data. In addition to the usual challenges (ill-posed data fitting), GISAXS introduces distortions and multiple-scattering effects. Because of the shallow angles, the x-ray beam is measurably refracted when it crosses the ambient-film interface; scattering is also refracted as it exits the film. These effects cause the detector image to be a 'distorted' version of reciprocal-space (stretched/compressed along $q_{z}$ ) [358, 371, 372]. The reflection-mode geometry leads to beneficial intensities; yet these enhancements can make quantitative crosscomparisons difficult $[373,374]$. For example, in GISAXS one observes an intense horizontal band of intensity on the detector (which can help to highlight weak signals). This Yoneda [375] (or Vineyard [376]) scattering occurs when the exit angle matches the critical angle (where waveguide modes are greatly increasing the probability of a scattering event). It can be difficult to quantitatively account for the enhancement factor. Finally, multiple scattering must be considered. In addition to scattering from the direct beam, one also has scattering from the reflected beam. Moreover, the scattering itself can either directly reach the detector, or be reflected first. These four possible scattering events appear as two scattering images on the detector [358]. That is, each scattering peak will appear twice (shifted along $q_{z}$ ); these peaks may also overlap, giving rise to elongated peak shapes. Measurements at multiple angles can resolve whether a given peak arises from the direct beam or the reflected beam, since the latter shift as the reflection angle is changed. These four possible scattering events (really the first four terms in an infinite series of progressively more complex but less likely multiple-scattering combinations) can be accounted for theoretically. The first term (direct scattering) is known as the 'Born Approximation' (BA), whereas accounting for the first four terms can be done using the 'Distorted Wave Born Approximation' (DWBA) [376-378]. A variety of elegant DWBA variants have been created to account for a variety of experimentally-relevant configurations [371, 379-382]. With care, one can quantitatively fit GISAXS data (some software packages now exist for the purpose [367, 383-389]).

Several alternatives to GISAXS have been described. One can use a series of transmission-mode measurements, across a range of sample angles, to reconstruct the same slice of reciprocal-space probed in GISAXS. This technique has been termed critical dimensions SAXS (CD-SAXS) [390-395], in analogy to the CD-SEM method for quantifying the order of lithographic structures. CD-SAXS has enabled precise measurement of lithographic structures [396-399], nanoimprinted polymers [400, 401], and block-copolymers [402, 403]. CD-SAXS is unencumbered by reflection-mode distortions, enabling precise fitting to measure detailed aspects of nanostructure (size, shape, LER, etc.). For instance, in BCP materials it has been shown how the morphology slightly distorts in order to accommodate guiding chemical templates. Similar experiments can be performed using neutrons; this 'rotational' scattering technique (RSANS) has been used to measure the ordering of BCPs [53, 310, 404, 405]. In GISAXS mode, one can rotate the sample in-plane, in order to reconstruct reciprocal-space. This can be used to measure single crystals [406], or the orientational spread in aligned samples [211, 407]. Recently, an alternative to GISAXS has been presented, which can obtain undistorted data 
without requiring lengthy reconstruction. Grazing-incidence transmission small-angle x-ray scattering (GTSAXS) [408-410] involves aligning the incident x-ray beam on the downstream edge of a sample/substrate, rather than directing the beam towards the center of the sample as typically done in GISAXS. This simple change enables the sub-horizon scattering (absorbed by the substrate in GISAXS) to escape and be recorded. By using a slightly larger incident angle $\left(\sim 1^{\circ}\right)$, GTSAXS can record an image without refraction-distortions or multiple-scattering complications. Data can be fit using the Born approximation, bypassing the complications of DWBA. The main downside is that one must arrange for the sample of interest to be positioned near the edge of the substrate. Spin-cast films often have edge effects, which make these regions poor representations of the average structure. On the other hand, it is usually trivial to dice a substrate so as to expose an edge of representative material. It has been found, experimentally, that dicing does not introduce problematic changes to the structure of most nanomaterials.

An exciting development in the study of soft materials is the availability of soft $\mathrm{x}$-ray beamlines. $\mathrm{X}$-ray energies of $0.2 \mathrm{keV}$ to $1 \mathrm{keV}$ allow access to the $\mathrm{x}$-ray absorption edges of $\mathrm{C}, \mathrm{N}, \mathrm{O}$, etc. This resonant soft $\mathrm{X}$-ray scattering (RSoXS) [411-414] can greatly enhance the scattering contrast between otherwise similar soft-matter components. Moreover, experiments at multiple energies can adjust the relative contrast of different constituents in multi-component systems, allowing components to be selectively contrast-matched ('made invisible'), and overall allowing the reconstruction of multi-component nanostructures. Wang et al. demonstrated how RSoXS could be used to unambiguously reconstruct the morphology of a triblock copolymer [17]. Sunday and Kline used resonant reflectivity to probe the interfacial width in BCPs [281]. These instruments also offer the possibility of probing molecular bond orientation, simultaneous with resolving nanostructure [415]. As soft x-ray beamlines develop, we can expect yet more powerful capabilities to become available [357].

An emerging trend in block-copolymer materials is to generate hierarchical, multi-scale patterns, where self-assembly generates nanoscale order within domains defined using a larger-scale technique. Conventional lithography allows hierarchical control via substrate patterns [73, 416422] or nanoimprinting [423-426]. Annealing can be controlled locally using a scannable nozzle releasing solvent vapor [427], or with laser annealing in a raster-scan [428] or masked mode [57]. Controlled wetting [361, 429] or evaporation [430] can define film regions within which the BCP orders. Onses et al. demonstrated the use of jet printing to define hierarchical BCP patterns [431, 432]. These hierarchical materials create a corresponding measurement challenge: to simultaneously measure structure at many length-scales. X-ray scattering beamlines are increasingly working to offer micro-focused and nano-focused x-ray beams [433, 434]. Small beams can be used to generate realspace maps of samples [435], where each pixel can contain a scattering dataset encoding nanoscale and molecular-scale information. Coherent imaging methods are also improving, promising to allow direct imaging of materials at many lengthscales [436-438]. 


\section{Ordering Using Plasticizing Additives}

Block-copolymer assembly is regulated by a delicate balance of energetic effects, especially the chemical mismatch of the block components, and chain stretching effects. Thus, a crucial means of controlling their ordering is to tune the interaction energies within the system.

\subsection{Solvent vapor annealing}

In solvent vapor annealing (SVA), a block-copolymer thin film is exposed to a vapor that is either a good solvent for both blocks, or a preferential solvent for one of the blocks. This solvent uptake induces swelling, plasticizing, and thus enhances ordering of the BCP morphology. The mechanism of rapid ordering under SVA with non-preferential, good solvents can be directly compared to thermal annealing, since both methods reduce the polymer viscosity and increase the mobility of the polymer chains. The SVA mechanism can be considered thermodynamically equivalent to thermal treatment, and mapped onto the BCP phase diagram as a decrease in the effective $\chi N$ segregation parameter (Figure 7a); or as a kinetic effect, lowering the $T_{g}$ of the system [439-441]. As reviewed by Hillmyer et al. [442], SVA can be conducted in various experimental setups (Figure 7b-d), but even in its simplest implementation-a closed 'jar' with a liquid solvent reservoir-SVA is very effective in accelerating the ordering of BCP thin films [362]. Despite its superficial simplicity, SVA can be quite difficult to control reproducibly, with results dependent on experimental factors such as chamber size and geometry, the location of the solvent reservoir, precise temperature control, and relative humidity. Moreover, care must be taken to avoid excessive swelling ratios or swelling rates, which can induce film dewetting, structural rearrangement, or topographic reconstruction [443-445]. While it is relatively straightforward to control swelling ratio (with online thickness monitoring) and exposure time, SVA ordering and morphologies are also strongly dependent on kinetic aspects such as the swelling rate, the quench rate, and the sample extraction protocol [446-452]. Solvent removal rate is particularly important for the ordering of cylindrical systems when a vertical orientation is desired, as the propagation of the drying front through the film thickness defines the ordering direction of the cylinders $[448,453]$. The need for precise control of SVA parameters has driven the development of more sophisticated, multi-point thermostated, flow-through setups (Figure 7c), which permit independent control of the partial pressure and flow-rates of multiple solvents. These setups benefit from real-time closed-loop control over the film swelling ratio and morphology (through the use of interferometry, ellipsometry, or GISAXS) [54, 365, 454-457]. Despite these advances, kinetic data for BCP grain-coarsening under SVA conditions are scarce. Cross-comparison of SVA results is complicated by inconsistencies in definitions of the annealing duration (e.g. with or without an equilibration period), and when swelling or deswelling rates are unreported. Stein $e t a l$. observed an order-of-magnitude faster grain-growth for PS- $b$-PEO annealed inside a small volume chamber $v s$. a large one, owing to differences in saturation time [458], which illustrates the difficulties inherent to hardware design, experimental reproducibility, and cross-comparison of results. Nonetheless, it can generally be concluded that in common BCP systems, a one-hour solvent vapor anneal can easily give rise to $\xi>1 \mu \mathrm{m}$, which corresponds to a more than 10x improvement over oven annealing (PS- $b$-PMMA [459], PS- $b$-P2VP [460], PS- $b$-P4VP [362, 461-463], PS- $b$-PDMS [464, 465], PS- $b$-PEO [453, 458, 466], PS- $b$-PLA [447, 467]). SVA is one of the few methods suitable for ordering of high 
molecular weight systems, which exhibit extremely slow pattern-formation kinetics. Kim et al. [459] used a simple bell-jar, sub-ambient temperature annealing approach to order lamellar PS- $b$ PMMA of total molecular weight $M_{n}=256 \mathrm{~kg} / \mathrm{mol}$ (with defect density decreasing as $\rho_{d} \sim t^{-1.35}$ ). In combination with topographic confinement, ordering of systems with $M_{n} \sim 1 \mathrm{million} \mathrm{g} / \mathrm{mol}$ is possible. It is worth noting that SVA-processed films often require additional oven annealing to remove film wrinkles resulting from buckling during deswelling [452].

Generally speaking, ordering kinetics increase with solvent uptake. However, increasing the film swelling ratio risks crossing the solvent-defined ODT [441], erasing any existing order, or inducing film dewetting from the substrate [444]. One can avoid crossing ODT by using binary solvent mixtures where one solvent is preferential, since this increases $\chi$ and stabilizes the phaseseparated state $[54,456,458]$. Condensation of bulk solvent on the polymer film (which leads to dewetting) can be avoided by increasing the temperature of the film relative to the solvent vapor; however this is ineffective as it very strongly shifts the equilibrium towards the dry state. Some groups have conditioned their films at sub-ambient temperatures [459], which increases solvent uptake but risks catastrophic solvent condensation if the film-vapor temperature differential is too high. Warm $\left(\sim 60^{\circ} \mathrm{C}\right)$ solvent vapor was found to increase ordering kinetics; for cylinderforming PS- $b$-PDMS in a trench pattern, the ordering time was reduced by more than an orderof-magnitude (to $30 \mathrm{~s}$ ), compared to room-temperature SVA at the same swelling ratio $\left(\rho_{d} \sim t^{-1}\right.$ at $23{ }^{\circ} \mathrm{C} v s . t^{-3}$ at $60^{\circ} \mathrm{C}$ ) [457]. A high-temperature combined with high-pressure approach to SVA has been proposed by Buriak et al., where a sealed pressure-resistant annealing vessel was heated using microwaves (Figure 7d). The heating simultaneously increases the temperatures of the solvent and the substrate beyond the solvent boiling point, and increases pressure to several atmospheres. This provides a spectacular enhancement of the ordering kinetics in high molecular weight systems, without epitaxial guiding [117, 468].

The substantial kinetic acceleration of SVA has been coupled with other DSA methods to resolve the problem of the poly-grain ordering of BCP domains. Long-range ordering has been demonstrated when SVA is combined with graphoepitaxy [117, 454, 463, 465, 469] and chemoepitaxy [361, 460] or swelling-induced soft-shearing methods [292, 427, 461] (Figure 7e).

\subsection{Plasticization during film casting}

Plasticizing and ordering solvent effects can be also exploited at the stage of film casting. For example, the fast evaporation of a selective solvent can balance the chain relaxation rate and microphase separation driving force, thereby inducing rapid development of vertical cylindrical domains [448]. Perego et al. showed that toluene trapped in a neutral brush underlying a PS- $b$ PMMA film plays a role in enhancing subsequent ordering kinetics [470]. Such results, combined with the fact that as-cast polymer films may retain residual solvent [471], underline the necessity to scrutinize experimental protocols for BCP annealing, in particular when crosscomparing kinetic data. Ordering kinetics can also be increased by changing the temperature of the film casting. In warm $\left(40-60^{\circ} \mathrm{C}\right)$ spin-coating experiments, Lee et al. observed quick graphoepitaxial ordering of a low molecular-weight BCPs without additional annealing [472]. 


\subsection{Direct solvent immersion}

Direct immersion annealing (DIA, iDSA) is an alternative, and arguably more convenient, way of delivering small-molecule plasticizers into BCP films. In this method, the BCP film is immersed in a solvent mixture that plasticizes the BCP, without dissolving the film. Park et al. [473] demonstrated ordering of a high- $\chi$ PS- $b$-PDMS by combining DIA with nanolitographic guide patterns. Modi et al. [474] recently used DIA to investigate the optimal conditions and ordering kinetics of PS- $b$-PMMA thin films. The two-solvent mixture, consisting of a nonsolvent (heptane) and a selective solvent (acetone), is chosen based on the solubility parameters of the pair and, at relatively modest swelling-ratio, facilitates increased mobility of the chain while sustaining sufficiently high $\chi$ to prevent phase-mixing. Interestingly, the acceleration of the grain-growth kinetics manifests as a large increase in the pre-exponential factor $(k)$, compared to the value for oven annealing [56], while the kinetic exponent $(\alpha)$ remains relatively small.

\subsection{Blending methods}

The composition of a BCP film can strongly influence ordering kinetics. For instance, cylinder BCP phases order more rapidly than lamellar, owing to chain diffusion [222, 475], while novel block-polymer architectures can potentially enable vastly improved grain sizes [476]. Blends of block-copolymers [87-95] can give rise to novel structures [28, 199], and blending can also influence ordering kinetics. Blending species into a BCP phase can affect the Flory interaction parameter $(\chi)$ [477-480]. Neutral additives should reduce segregation, effectively plasticizing the material and accelerating assembly kinetics. Conversely, blending additives that have a selective affinity for one of the blocks has been demonstrated to increase segregation strength [282, 480483], which increases ordering quality (e.g. sharpening interfaces) but also increases ordering timescales. Blending of short neutral (random copolymer) chains has been suggested as 'melting' local defects, thereby improving ordering [484]. Nanoparticles can be blended into BCP systems to create new hybrid structures [79]. Blended nanoparticles may localize to defective film regions (such as grain boundaries), stabilizing them and thereby influencing ordering kinetics [485]. The kinetics in hybrid systems may be highly non-trivial; for instance, there may be separate assembly timescales for BCP phase-separation, and the ordering of the nanoparticle fillers [486].

\section{Ordering Using Interfacial Effects}

Thin films are generally known to be strongly influenced by both the nanoscale confinement, as well as the presence of the film-substrate, and film-ambient interfaces. The order of BCP thin films, in particular, is strongly influenced by the interfacial energies at the substrate and top surfaces [487].

\subsection{Substrate-mediated ordering}

Substrate surface chemistry influences BCP assembly. Substrates with strong interaction with one of the blocks will introduce preferential wetting, which in turn templates the type and orientation of the morphology. For example, lamellar phases will orient horizontally (with the lamellar sheets parallel to the substrate plane). On the other hand, 'neutral' surfaces can be created whose surface energy is intermediate between the two blocks [75, 259, 488-492]. A 
robust method is to graft a random copolymer brush to the substrate, with the brush composition roughly matched to the relative volume fraction of the two BCP blocks. Alternatively, intermediate surface energies can be obtained by controlling grafting density [493], controlling the ratio of species reacting with [494-496] or assembling on [497, 498] a substrate, or partially oxidizing certain hydrophobic substrates [310, 404, 499-503]. By establishing roughly equal interaction strength with the two blocks, there is no net preferential segregation. Although one might expect 'undirected' ordering (random orientation distribution), surface entropy instead promotes preferentially vertical orientations of morphologies [236]. For instance, cylinder phases on preferential substrates orient horizontally (long-axis parallel to substrate plane), whereas on neutral substrates they orient vertically (perpendicular to substrate). More generally, there is an interplay between substrate-directed and confinement-driven ordering, which can tune orientation [273, 504].

The strength of substrate interactions influences ordering kinetics. Generally speaking, a strong substrate interaction will slow kinetics, while unfavorable substrates will increase ordering kinetics. On a strongly-adhesive substrate, polymer molecules become pinned and diffusion is arrested [505]. Reducing the substrate interaction improves ordering. Harrison et al. [301] found that grafting a brush layer to the substrate with the same composition as the BCP majority phase yields a 'self-lubricating layer,' increasing the ordering rate, as compared to the same BCP on the native oxide of the silicon substrate. This effect is less pronounced when it is the minority block that interacts strongly with the substrate. This effect can be further enhanced by selecting a substrate-brush material that is dissimilar to the BCP phases. Quach et al. [191] systematically varied the $\mathrm{BCP}$-substrate interaction parameter, and found that large incompatibility between $\mathrm{BCP}$ matrix and substrate brush (i.e. large $\chi_{\text {matrix-brush }}$ improved order.

A more extreme version of substrate chemical control is to use chemical patterns [506, 507], typically produced using lithography (chemoepitaxy $[75,508]$ ). In this case, the two block components will segregate locally, registering with the substrate pattern and templating off of it. This can be used not only to enforce orientation (especially vertical), but to exactly enforce local positioning of both blocks. If the chemical template is ordered over macroscopic areas, then the templated BCP morphology is similarly ordered macroscopically. Chemoepitaxy is typically coupled with thermal annealing, which enables local phase separation, and morphological rearrangement in order to follow the guiding pattern. For a 1:1 chemical template, rearrangement need only occur over distances of $\sim L_{0}$, which leads to very fast ordering times (minutes). Edwards et al. studied in detail the ordering of a lamellar BCP on a chemical line pattern [509], identifying several intermediate states. That is, the BCP first generated hexagonally-packed domains (reminiscent of vertical cylinders), which aligned with the chemical template. These domains then fused into defective lamellar lines, with defects gradually disappearing. Notably, elimination of topological defects in this case does not require the usual diffusion and annihilation; instead, defects can be spontaneously eliminated through BCP rearrangement. Thus, the strong driving force of the underlying chemical pattern enforces order. This rapid convergence to near-perfect macroscopic order can be considered as an enormous acceleration of the self-assembly process. 
Substrate topography can strongly influence self-assembly, especially by selecting the in-plane and out-of-plane orientation of the BCP morphology. This has been investigated with respect to roughness [53, 277, 310, 404, 510-512], nanoparticle monolayers [513], faceted substrates [469, $514,515]$, corrugated surfaces [278, 516, 517], film-spanning particles [276, 518], and guiding trenches [519-523] (graphoepitaxy [73, 74]). In many graphoepitaxy schemes, a BCP is ordered within a trench, with the morphology rapidly aligning with the trench sidewalls (if the trench width is commensurate to the $\mathrm{BCP}$ repeat-spacing), or against the trench length (if the trench is incommensurate). As with chemical patterns, the formation of a BCP phase with uniaxial orientation, and even well-defined registry over macroscopic distances, represents an enormous acceleration compared to conventional oven annealing. On the other hand, one must keep in mind the labor costs of DSA methods; that is, the time spent creating the template itself. The advanced optical or electron-beam lithography used to generate the underlying pattern may require multiple stringent processing steps. Nevertheless, the degree of order and control over material registry (both locally and macroscopically) allows these DSA methods to access regimes not available with any other techniques.

\section{2. $\quad$ Free surface-mediated ordering}

Ordering of block-copolymer thin films is strongly influenced by the free surface. In general, one of the two block materials will have a lower surface tension, and will be preferentially segregated to the top surface. This propensity will then drive ordering through the depth of the thin film. For instance, this difference in surface tension generically drives lamellar phases to orient horizontally (lamellar planes parallel to substrate). Formation of robust vertical BCP orientations thus requires neutralization not only of the substrate interface, but also of the free surface. For some BCP materials, the temperature-dependence of the two surface tensions may cause them to coincide at some specific temperature. Annealing at this temperature thus removes the propensity for preferential surface segregation. For instance, polystyrene-block-poly(methyl methacrylate) (PS- $b$-PMMA) orients vertically $[488,492]$ near $230^{\circ} \mathrm{C}$. Interfacial neutrality alone does not guarantee a vertical morphology orientation, since neutrality merely removes the preferential orientation induced by one component wetting an interface; i.e. one would naively predict that neutrality leads to a completely random and unbiased orientation distribution. However, the BCP chains and morphology are not blind to the discontinuities of interfaces. Forrey et al. used molecular dynamics simulations to show that a surface entropy field drives chains to orient with their long-axis in the interfacial plane, and thus promotes a vertical orientation of the $\mathrm{BCP}$ morphology [236].

The energetics of a thin film top surface can also be regulated by applying a neutral 'topcoat' material [524-529]; this strongly selects the perpendicular orientation, with well-defined morphologies forming on minute timescales. Zhang et al. demonstrated that this topcoat can be embedded within the BCP material [530]. That is, a polymeric surface-active additive can be added to the BCP solution that is cast. During annealing, this material spontaneously segregates to the top surface, generating a neutral interface and thus controlling film orientation. This strategy thus enables orientation control and rapid annealing, without introducing an additional film-application step. 


\subsection{Interplay of substrate and surface interactions}

Of course, the overall ordering of a BCP film is influenced by the combined effect of the substrate and top surface driving forces. The interplay between surface energies (top and bottom interface) and film thickness were investigated in detail by Durand et al. [531] for the case of lamellar morphologies. Film thicknesses commensurate with an integer number of lamellar layers preferentially form horizontal/parallel orientations; similarly, preferential surface wetting promotes horizontal states. However, incommensurate confinement instead promotes perpendicular orientations; mixed phases can develop when commensurability and surface interactions are in opposition.

For an unconfined thin film, when both interfaces are preferential, lamellar phases generate horizontal lamellae, and further generate islands/holes (of height $L_{0}$ ) for incommensurate thicknesses. Kim et al. [259] demonstrated that when the neutrality of the top and bottom interfaces are in opposition, the islands/holes instead have a height of $L_{0} / 2$. For a preferential substrate but a neutral top surface, the surface-expressed block alternates between the high and low regions (enabling the $L_{0} / 2$ height step). The reverse condition-neutral substrate but preferential top surface-involves high and low regions that have different blocks wetting the buried substrate interface. This suggests an elegant way to direct BCP ordering: by carefully balancing commensurability, and interfacial effects.

\section{Ordering Using Fields}

External fields alter the process of self-assembly by imposing energetic constrains on the nucleation, growth, and stability of BCP domains. In particular, fields break symmetry by changing the relative energies of domains with different orientations [50].

\subsection{Electric and magnetic fields}

For electric and magnetic fields, the energetic bias originates from the anisotropy (contrast) of dielectric permittivity $(\Delta \varepsilon)$ or magnetic susceptibility $(\Delta \chi)$ between the chemically-distinct BCP blocks $[67,71]$, and is proportional to the square of the applied field ( $E$ or $B$, respectively). The thermodynamic driving for alignment, $\Delta E$, is also affected by the volume of domains with coherent order $(V)$ :

$$
\Delta E=\frac{V B^{2} \Delta \chi}{2 \mu_{0}}
$$

where $\mu_{0}$ is the vacuum permeability. In a typical Gaussian-coil BCP, both the magnetic and electric anisotropies are relatively low, and the intrinsic domain sizes are small. Thus, rather large fields are required to induce morphological alignment. As electric interactions are much stronger than magnetic ones, field-alignment of BCPs was first successfully demonstrated using static electric fields $[532,533]$. The weaker driving force of magnetic fields is suitable for the alignment of bulk and semi-thin samples such as membranes [69, 70,534]; that is, materials not dominated by surface-ordering effects [535]. The alignment direction depends on the sign of the anisotropy of a particular system. In most coil-coil BCPs with cylindrical or lamellar 
morphology, this direction is parallel to cylinder long-axis or the lamellar plane, inducing 'vertical' orientation of the domains when the field is applied in the substrate-normal direction. Under this alignment geometry, there is no energetic bias for the in-plane direction; the in-plane energetic degeneracy thus leads to a '2D-powder' (poly-grain) alignment. Nonetheless, this ordering geometry is commonly used in electric field-mediated self-assembly of BCP films due to the ease of the field application (parallel-plate condenser), and because it enforces continuity of the BCP domains through the entire film thickness [144, 536]. Conversely, an in-plane alignment of BCP cylinder or lamellar domains on neutral substrates is non-degenerate. The morphology aligns along the field, resulting in a pattern of parallel lines in the field direction [537, 538].

Field-mediated ordering is, in principle, a thermodynamic phenomenon that applies an orientational bias to the free energy landscape, as opposed to altering the grain-growth kinetics or ordering pathway. The kinetics of ordering are primarily dictated by the viscous forces impeding the reorientation of ordered grains in the polymer melt. The characteristic ordering timescale, $\tau$, is proportional to the viscosity, $\eta$, which in turn depends exponentially on temperature [539]:

$$
\begin{aligned}
& \tau \propto \frac{\mu_{0} \eta}{B^{2} \Delta \chi} \\
& \eta=\eta_{0} \exp \left[E_{a} / k_{B} T\right]
\end{aligned}
$$

where $E_{a}$ is the viscous flow activation barrier, and $\eta_{0}$ is a phenomenological variable. Given the immobility of polymer chains below the glass transition temperature, electric or magnetic field alignment of BCPs is performed at high temperatures, well-above $T_{g}$. The ordering timescales are regulated by the magnitude of the driving force (field strength, anisotropy), the size of coherently-ordered domains, and the viscosity of the system. Rapid ordering and alignment is observed in systems with accessible order-disorder transition temperatures, such as low molecular-weight or liquid-crystalline (LC) materials. These materials also possess intrinsically large grains with high anisotropies. Ordering upon cooling from the mixed into the microphase separated state can occur on timescales of minutes, provided that the optimal thermal processing window is known [540]. Surprisingly, this approach was recently shown to be effective even in the magnetic alignment of a coil-coil PS- $b$-P4VP system, with purely-intrinsic magnetic susceptibility anisotropy [342]. Sub- $T_{\text {ODT }}$ reorientation, or alignment of nucleating grains in ascast films, is typically much slower due to the high viscosity of a polymer melt. Electric-field alignment times for coil-coil BCPs from an as-cast melt are of the order of several hours [532], even when combined with a plasticizing vapor atmosphere [538]. In comparison, concentrated BCP solutions can be aligned in minutes [541]. These timescales are of the same order-ofmagnitude as those observed by SAXS for magnetic-alignment of bulk liquid-crystalline BCPs, which proceeds through a grain rotation mechanism [539]. This again highlights the importance of viscosity in field-mediated alignment. The alignment kinetics can also be improved by modifying the material's propensity for field-alignment by blending in a low molecular-weight anisotropic plasticizer, thereby increasing the susceptibility contrast $(\Delta \chi)$ [542]. In the case of 
electric field experiments, sequestering metallic nanoparticles into one of the blocks increases the permittivity anisotropy $(\Delta \boldsymbol{\varepsilon})$, and improves field-alignment [543].

\section{2. $\quad$ Shear fields}

Shear was historically one of the first methods used to orient lamellar or cylindrical morphologies in bulk block-copolymers [544, 545]. An advance towards shear-ordering of thin films was made by Angelescu et al., who used a conformal layer of crosslinked PDMS to transduce the shear force to a heated BCP film [60]. The technique has been extended to shearalign various block-copolymer systems with different morphologies: spheres [546], cylinders [547], and lamellae [548]; frequently with the use of rheometric setups and high-viscosity silicone oils instead of elastomeric PDMS pads as shear transducers [549]. The timescales for alignment in shear experiments, as measured by the evolution and saturation of the orientational order parameter, are reasonably short. Typically, shear stress is applied to a sample heated above $T_{g}$ for $\sim 30 \mathrm{~min}$, followed by cooling to room-temperature to immobilize the material $[547,550]$. A key advantage of shear-alignment is the relatively simple experimental setup, combined with the ability to order materials over arbitrarily large areas. Thus the 'total cost' of shear ordering (both ordering time and sample preparation time) is quite low. Shear-aligned BCP patterns have been used as templates for fabrication of large-area single- and double-layered arrays of inorganic nanostructures $[112,139,141,551]$.

While shear-alignment offers a rapid way of obtaining globally-aligned monolayers composed of horizontal cylinders or vertical lamellae, the near-ideal line patterns are compromised by the presence of relatively sparse dislocation defects and characteristic periodic morphology undulations [550]. There is a well-recognized, negative correlation between the number density of dislocations and the orientation order parameter. Even in 'defect-free' (within the microscopic observation field) samples, the orientational order parameter does not reach unity, due to the presence of small-amplitude undulations. Although the order in a film can be optimized through selection of film thickness (optimum near $1 \mathrm{~L}_{0}$ ) [60], or by fine-tuning the block ratio [547], the presence of undulations may, in some regimes, be an intrinsic feature of shear-ordering.

\section{Ordering Using Advanced Thermal Methods}

Although a variety of elegant directed assembly strategies have been described, purely-thermal annealing methods remain attractive from the point of view of simplicity. As such, a variety of research programs have investigated the limits of thermal ordering of BCPs. An obvious approach is to increase the annealing temperature. Rapid thermal processing (RTP) typically involves irradiating a sample with an infrared light-source. This allows high temperature to be rapidly achieved, since the sample is directly heated (photo-thermally). As previously mentioned, RTP has been applied to BCP thin films, where rapid heating to high temperature (up to $350^{\circ} \mathrm{C}$ ) [237, 245, 246] led to rapid ordering (and formation of nanostructure before the onset of polymer degradation). Another convenient method for rapidly delivering energy to $\mathrm{BCP}$ samples is to use microwave heating. BCP films can be heated alone [552, 553], or annealed in combination with solvent exposure and/or graphoepitaxial templates [117, 468, 554]. In this method, the final order can depend strongly on the heating rate, and the position of the sample within the microwave 
field [553]. The microwave radiation may be absorbed by the silicon substrates (if the Si wafer is sufficiently doped) [555], or may be absorbed by polar solvent species [556]. Heating polymer films through irradiation allows for rapid heating and cooling, and thus controls the temporal thermal history (c.f. lower-right of Figure 2). However, one can also consider control of the spatial component of thermal processing.

In semiconductor manufacturing, moving thermal zones are now routinely used to generate highly-ordered atomic crystals of inorganic matter. Originally developed by Pfann, zone melting consists of moving a hot melting zone through a material; impurities will segregate into the molten region, and thus the moving melt zone sweeps away impurities, leaving behind a pure material ('zone refinement') [557]. A related phenomenon is directional solidification, where a particular crystal orientation is templated by existing solid, such that a moving thermal zone leaves behind a single-crystal material. The application of zone heating methods to organics is more recent [558]. Lovinger et al. used temperature gradients to control polymer crystallization [559]. Hashimoto et al. applied moving thermal gradients to bulk block-copolymers (Figure 8a), where they demonstrated the ability to form extremely large grain sizes [560-566]. In the socalled 'hot zone annealing' (HZA) conditions, the polymer film is heated above the disordering temperature $\left(T_{\mathrm{ODT}}\right)$; and morphology develops on the cooling front through a directional solidification process $[567,568]$. In addition to the influence of the spatial thermal gradient, substrates can play a critical role in determining morphology orientation [565]. Angelescu et al. used a microfabricated heater to induce directional solidification in a BCP monolayer, demonstrating greatly improved grain sizes [569]. For BCP materials, it is not always possible to access $T_{\mathrm{ODT}} ;$ e.g. this temperature may be above the polymer degradation temperature. As a result, a parallel research thrust has investigated 'cold zone annealing' (CZA), where the polymer film is heated above $T_{g}$ to induce mobility and thus coarsening of morphology, but remains below $T_{\mathrm{ODT}}$ and thus within the ordered region of the phase diagram at all times (similar to oven annealing). Berry et al. developed an apparatus for CZA processing of BCP thin films (Figure 8b), and discovered that the zone direction induced a weak alignment of the BCP morphology, and that zone annealing led to enhanced ordering kinetics ( $\alpha=0.46$, compared to the usual $\alpha \approx 0.25$; Figure 4 compares the scaling for CZA vs. oven annealing). The alignment is unexpected given the lack of a well-defined interface (e.g. solid-liquid or order-disorder). The enhanced kinetics were studied by generating 'frozen zone' samples; that is, by interrupting the zone annealing process, such that the entire annealing history is encoded within the sample as a function of position (relative to the thermal zone at the moment annealing was terminated). These studies found the enhanced kinetics to be related to the in-plane thermal gradients [229]. Yet it is surprising that macroscopic thermal gradients $\left(\nabla \mathrm{T} \approx 17^{\circ} \mathrm{C} / \mathrm{mm}\right)$ are able to radically influence nanoscale $\left(L_{0} \approx 26 \mathrm{~nm}\right)$ self-assembly. Self-consistent field (SCF) simulations have investigated the role of dynamic heterogeneities [570] or spatial variation of polymer mobility [571, 572]; while these studies capture important physics, the size-scale of the simulated gradients are orders-of-magnitude sharper than those produced experimentally.

CZA methods have been progressively refined, including generating sharper gradients ( $\nabla \mathrm{T} \approx 45^{\circ} \mathrm{C} / \mathrm{mm}$ ) [573], to control BCP orientation [573], coupled with graphoepitaxy [574], in nanoparticle-BCP composites [575, 576], for mesoporous materials [123, 577], and using a 
rotational apparatus to generate a rotating thermal zone [578]. A particular exciting demonstration - especially from the point of view of industrial scale-up of self-assembly - is the integration of CZA methods into roll-to-roll processing (Figure 8c) [579, 580].

Recently, dynamic annealing methods have been explored in a photo-thermal mode. Majewski and Yager developed a laser zone annealing (LZA) setup, wherein a laser beam is focused into a line at the surface of a BCP thin film (Figure 8d) [57]. The substrate is coated with a thin lightabosrbing layer $(\mathrm{Ge})$, such that the moving laser line generates a moving hot zone. The tight laser focus allows for extreme thermal gradients $\left(\nabla \mathrm{T}>4,000^{\circ} \mathrm{C} / \mathrm{mm}\right)$, while the laser-based method more generally allows for detailed control the thermal history through the laser intensity, focus, sweep velocity, etc. This detailed control of spatio-temporal annealing history allows many aspects of self-assembly to be probed (Figure 2, upper-right). It was found that LZA processing enhances ordering kinetics by $\sim 1,000 \times$ compared to traditional oven annealing (whereas CZA yields a $\sim 10 \times$ enhancement compared to oven annealing), allowing for millisecond ordering (Figure 4 compares the scaling law for grain growth using various methods). Two factors play a role: the thermal spike allows rapid ordering (high peak temperature) before the onset of polymer degradation (similar to RTP annealing); and, the in-plane thermal gradient additionally enhances ordering kinetics. A possible mechanism is that the thermal gradient exerts a thermophoretic force on topological defects, driving them towards the hot region of the zone (where their energy is lowered). This net force on defects will both sweep them out of the sample during annealing (similar to zone refinement), and will increase the rate of defect annihilation (by concentrating defects into the high-mobility hot zone). When ordering temperatures are sufficiently high, photo-thermal zone annealing can spontaneously induce alignment of the BCP phase, due to shearing of the polymer material [57] and/or directional solidification [581, 582]. Photo-thermal annealing is a promising means for facile ordering of BCP materials. For instance, it has already been demonstrated that one can use graphene as the light-absorbing layer, allowing ordering on flexible and curved substrates [582].

A clever extension of zone annealing methods is to exploit the annealing zone to simultaneously shear-align the BCP film. Adding a soft elastic cladding to the BCP film (typically poly(dimethyl siloxane), PDMS) allows for spontaneous shearing during CZA, owing to the differential thermal expansion between the soft cladding and the rigid substrate. It has been demonstrated for both CZA [156, 407, 583, 584] and LZA [57, 119, 211, 585] approaches that this 'soft shear' (SS) mode allows the rapid formation of highly-aligned morphologies. For instance, SS-LZA can generate highly-aligned $\left(\sigma_{\phi}<1^{\circ}\right)$ BCP morphologies in $<10 \mathrm{~s}$. SS-LZA was also used to probe the fundamental origin of BCP response to shear forces [585], where it was found that the relaxation time of the BCP morphology $\left(\tau_{r}\right.$, Figure $5 \mathrm{~b}$ ) plays a critical role. Soft BCP materials with relaxation times smaller than the shearing time scale (inverse shear rate) will not respond to the shear field, since they relax and dissipate the strain field faster than it is generated.

Conversely, stiff materials with long relaxation times are amenable to shear-alignment. Materials with relaxation times roughly commensurate to the shearing time scale will be 'responsive' to the shear rate (i.e. alignment will depend on shear rate). Thus, while in static shear the ordering depends primarily on the shear stress [586], in dynamic shearing the shear rate plays a critical role [587]. 
Overall, thermal processing that is spatially heterogeneous and temporally dynamic provide a powerful means of controlling BCP ordering (Figure 2, upper-right), while employing relatively simple experimental apparatus (Figure 8). In general, these methods do not require exotic substrates or sample preparation, and thus are amenable to use in a manufacturing context. However, many questions remain unanswered regarding how self-assembly responds to nontrivial spatio-temporal thermal gradients.

\section{Discussion}

Figure 2 organizes the wide variety of BCP ordering methods according to the degree of spatial and temporal control. Oven annealing is the most straightforward method, but provides neither spatial nor temporal control of the ordering process. New techniques have pushed ever-further into providing more detailed control over the spatial environment and temporal history of ordering, and have thereby delivered improved (faster and more controllable) assembly. In comparing different methods of accelerating self-assembly, one must be cognizant not only of the ordering time itself, but also of additional costs of time and complexity associating with preparation of the substrate, sample, or experimental apparatus. For instance, epitaxial methods provide rigorous control of BCP assembly, and allow precise defect-free BCP patterns to be rapidly generated. However, the time associating with preparing the template must not be ignored. Similarly, BCP assembly can be controlled using interfacial energies, shear fields, dynamic annealing, etc.; however these methods require additional complexity with respect to sample preparation (e.g. special substrates) and annealing apparatus. Added complexity to the annealing apparatus is favorable in the sense that its construction is a one-time cost, whereas complex substrates incur a cost for every sample fabricated. In general, there is a tradeoff between the complexity of the ordering method, and the control the method provides.

There is a growing desire to transition self-assembling materials to an industrial context, where the issue of ordering timescales and processing complexity become extremely important. More complex ordering protocols are technologically undesirable owing to both increased cost, and decreased reliability. Many of methods described in the literature may not scale favorably to a high-throughput industrial context. For instance, solvent vapor (SVA) methods can be highly sensitive to processing conditions. Temperature variations vastly alter the film swelling ratio, and can even lead to catastrophic liquid condensation. This sensitivity to ambient temperature and temperature gradients may undermine the method in an industrial context. Conversely, preliminary results suggest that solvent immersion (DIA) may be a more industry-friendly alternative, accessing the same ordering mechanisms as SVA in a more robust manner. Electric and magnetic field alignment is relatively robust, and exploits well-established technology. However, the response of self-assembling materials to these fields is weak, thus limiting the industrial utility of these methods.

Industrial processing frequently benefits from being implemented as a continuous process (e.g. roll-to-roll) instead of a batch process. Whereas most annealing methods (oven, microwave, SVA, etc.) are a serial process, many newer methods (CZA, LZA, DIA) are very much amenable to roll-to-roll processing $[579,580]$. Grapho- and chemo-epitaxy require stringent lithography to 
form the guiding prepattern. In this sense, these methods are too expensive for fabrication of large quantities of ordered nanomaterials. On the other hand, these methods exploit the extremely mature and reliable infrastructure of modern micro-electronic manufacturing. It thus seems quite possible that BCP materials, ordered using lithographic templates, will play a role in future nano-electronics device manufacturing; in this context, the formation of a lithographic guiding pattern is obviously commensurate in complexity with other manufacturing steps.

Given the diversity of ordering methods, an obvious approach is to combine strategies; indeed combined annealing techniques are increasingly common. Solvent annealing techniques have been conveniently combined with other methods. A combination of SVA, thermal annealing $\left(220^{\circ} \mathrm{C}\right)$, and a neutral substrate demonstrated efficient $(\alpha=0.33)$ ordering [470]. Microwave annealing can be conveniently combined with SVA if solvent is included in the sealed processing vessel [117, 468, 554]. Epitaxial [588] or nanoimprint [425] methods can benefit from local solvent exposure. Local photo-thermal annealing can be performed in concert with solvent exposure to enhance ordering kinetics [428]. Vogt et al. have demonstrated that soft-shear (SS) alignment can be generated using solvent fields; that is, solvent uptake/evaporation induces swelling/contraction of a PDMS capping layer, which will shear an underlying BCP film [291, 292, 589]. This technique thus cleverly combines shear-alignment with SVA processing. Graphoepitaxy can be combined with CZA [574] or electric-field [590] alignment to enable synergistic ordering. It is likely that further combinations of ordering methods will yield new insights into the fundamentals of self-assembly (by comparing synergistic $v s$. antagonistic aspects), as well as further improvements in ordering kinetics. However, the increased complexity of combined approaches is not attractive in an industrial context.

The diversity of fast assembly techniques, as well as the impressively short ordering timescales that have been demonstrated, may lead one to believe that BCP ordering is a solved problem. However, as the boundaries of BCP ordering are pushed further, the issue of assembly time will reappear. For instance, there is considerable interest in materials that have a very large $\chi N$, which involves correspondingly arrested dynamics. High- $N$ materials are attractive for pushing selfassembly to larger lengthscales. High- $\chi$ materials are of great interest because this sharpens the domain interface (presumably decreasing LER in patterning applications), and allows access to smaller repeat spacings [591]. That is, with high- $\chi$ materials, one can use a smaller $N$ (with correspondingly smaller $L_{0}$ ) while still achieving proper phase separation at processing temperatures [592]. Similarly, introduction of blend components that increase $\chi$ will also slow the assembly kinetics. More elaborate polymer architectures (e.g. multiblocks) may also increase entanglements and hinder assembly. Thus, the kinetics of assembly remains a pertinent issue.

An emerging issue in the assembly of block-copolymers is the pathway-dependence of selfassembly. Although BCP ordering is often described in terms of the equilibrium phase diagram, and the associated equilibrium morphologies (Figure 1a), it is known that the structures observed experimentally are very often influenced by non-equilibrium aspects (kinetic trapping, metastable states, etc.). An example is shown in Figure 9a, which depicts the energy landscape for a BCP thin film. An as-cast polymer film is disordered, trapped in a high-energy configuration. Thermal annealing induces rapid phase separation, and the formation of a 
nanoscale morphology. However, this results in a large number of small grains with uncorrelated orientations. Such a poly-grain sample will coarsen very slowly, owing to the innumerable energy barriers in the self-assembly landscape. By comparison, if the initially disordered film is exposed to a rapid and intense shear force, long-range orientational correlations can be imposed before phase separation has appreciably begun [211]. This 'latent alignment' state has long-range (macroscopic) correlations, even though the nanoscale morphology has not yet developed. Thermal annealing of this state will rapidly generate a monolithically-aligned sample, since the material will simply descend into the adjacent global energy minimum. Thus, a particularly efficient ordering pathway for a BCP sample is to use an intense shear field to break symmetry, after which conventional thermal annealing will rapidly yield a highly-ordered material. This is of course a generic phenomenon; e.g. suitable application of an electric or magnetic field will similarly select an efficient ordering pathway.

Deng et al. used simulations to study the ordering of hexagonal patterns within confinement volumes [593]. By nucleating grain growth at the corners of a confinement volume, grains growing at great distances from one another are implicitly correlated, and thus merge to form a defect-free pattern (Figure 9b). Similar motifs have been observed experimentally in graphoepitaxy experiments; in general, careful preparation of guiding templates can thus select efficient and rapid ordering pathways.

An additional non-equilibrium effect to consider in BCP assembly is the appearance of transient states; that is, during BCP assembly one frequently observes configurations that do not persist if annealing is continued. While these transient states can be viewed as a nuisance, they can also be exploited. For instance, annealing can be terminated at an appropriate moment, thereby selecting a non-equilibrium morphology or orientation. Figure 9c shows an example of a cylinder-forming $\mathrm{BCP}$ thin films that undergoes two reordering transitions [238]. A disordered film initially forms randomly-packed vertical cylinders, which reorder into the expected hexagonally-packed cylinder domains. This well-ordered vertical cylinder state is also transient, with a reorientation transition eventually converting the system into a horizontal-cylinder morphology. With appropriate annealing protocol, the transient vertical-cylinder state could be selected, despite the lack of neutral substrate conditions. It was also observed that the final horizontal-cylinder state was influenced by the intermediate states; e.g. the final grain anisotropy is influenced by the heating rate during annealing. Overall, the growing number of examples of non-equilibrium selfassembly of BCP systems suggests an opportunity: by carefully designing the ordering pathway, desired structures (including non-equilibrium structures) can be selected.

In summary, a wide variety of methods have been devised for controlling the order of BCP materials. These methods can often control the type and orientation of the BCP morphology, either by directly templating it, or by more subtly biasing the BCP assembly energetics. Simultaneously, these methods have provided improved control over the self-assembly kinetics. Whereas BCP thin films typically require hours or days of annealing under oven annealing conditions, similar levels of order can now be achieved in seconds or even milliseconds. Moreover, modern BCP processing methods allow one to align the morphology, creating macroscopically-ordered materials in mere seconds. Assembly timescales have been accelerated 
to the point that wide deployment for certain applications now seems feasible. Nevertheless, continued progress is required in order to combine rapid assembly with the detailed structure control, and low defect densities, that high-performance applications demand. 


\section{Figures}

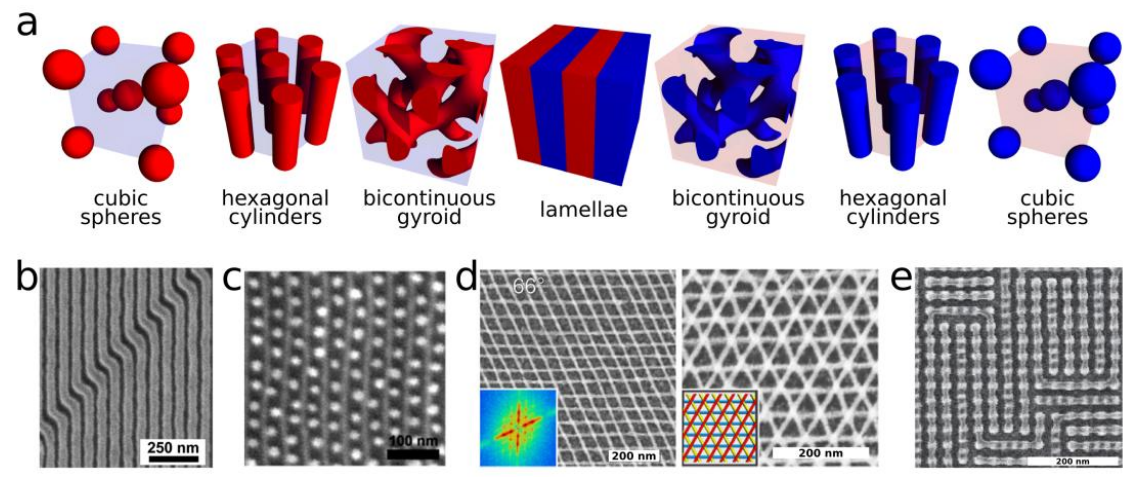

Figure 1: (a) Bulk equilibrium morphologies formed by diblock-copolymers. (b-e) Examples of more exotic structures that can be generated using BCP architectures. (b) Substrate chemical patterns can be used to direct BCP ordering, producing desired local geometries. Reproduced with permission from the American Chemical Society 2007. (c) BCP-derived patterns can be used to organize subsequent BCP materials [200]. Reproduced with permission from the American Chemical Society 2013. (d) BCP-derived nanowires can be layered to generate meshes of arbitrary symmetry [119]. Reproduced with permission from the Nature Publishing Group 2015. (e) Minimal topographic guiding patterns can be designed to enforce complex patterns in BCP assembly [185]. Reproduced with permission from the Nature Publishing Group 2014.

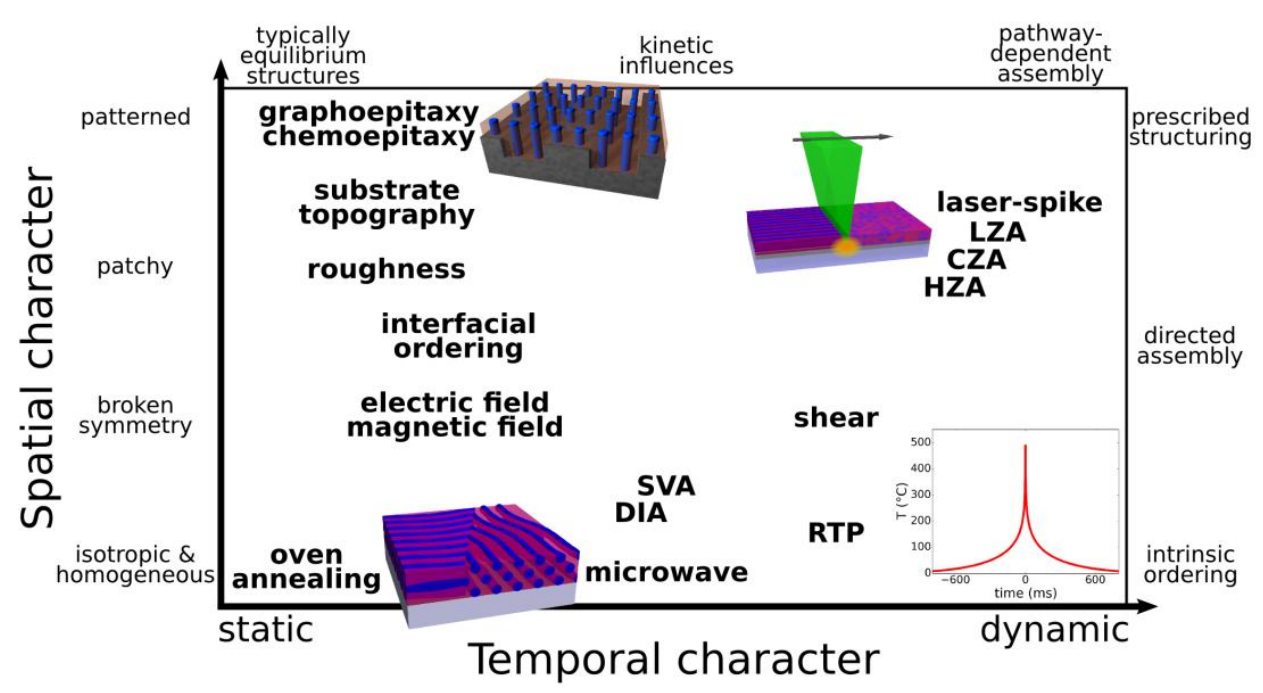

Figure 2: Methods for ordering block copolymers can be organized in terms of their spatial characteristics (homogeneous to patterned) and temporal character (static to dynamic). Oven annealing is spatially homogeneous, and temporally static (other than brief ramping/cooling phases). Solvent vapor annealing (SVA) and direct immersion annealing (DIA) are similar, though can be more sensitive to kinetic effects (e.g. quenching). A variety of directed self-assembly methods rely on breaking spatial symmetry, using fields, or substrate topography/chemistry. Techniques such as rapid thermal processing (RTP) use a dynamic thermal history (spatially-homogeneous thermal spike). Various zone annealing or local heating methods (possibly photo-thermal) exploit both dynamic thermal history (heating spike), and spatially non-uniform processing (spatial thermal gradients). This includes hot zone annealing (HZA), cold zone annealing (CZA), laser zone annealing (LZA), and laser-spike annealing. 

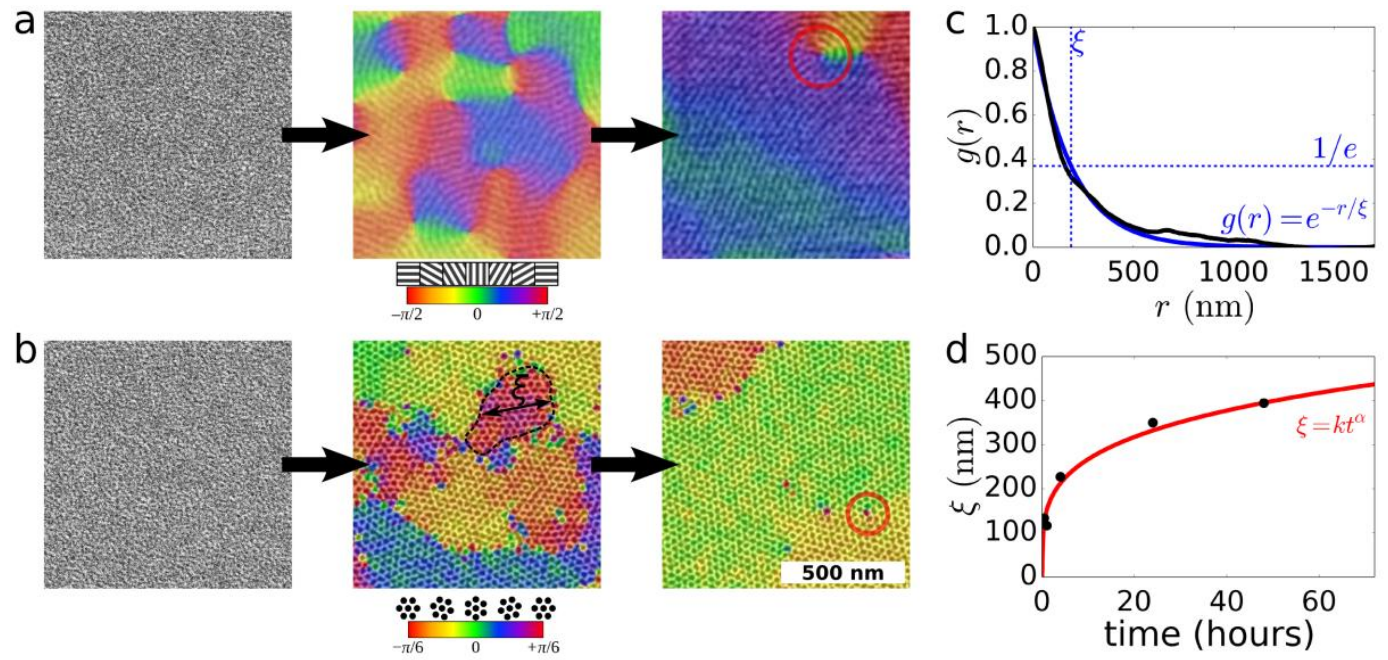

Figure 3: Examples of ordering behavior observed for BCP thin films. (a) Lamellar or horizontal cylinder morphologies appear as line patterns in microscopy (SEM or AFM), whereas (b) vertical hexagonally-packed cylinders appear as dot patterns. An as-cast film is initially disordered, but as annealing proceeds, well-defined morphologies appear. The local orientation of the morphology can be obtained using image analysis (color-coded in images). An example grain is highlighted by the dashed contour. Longer annealing times leads to larger domains of preserved orientation (larger grain sizes); though discrete topological defects may remain (red circles). (c) A quantitative grain size $(\xi)$ can be computed from the orientation data by fitting the orientation correlation function, $g(r)$, to an exponential decay. (d) Grain growth as a function of annealing time typically follows a power-law. 

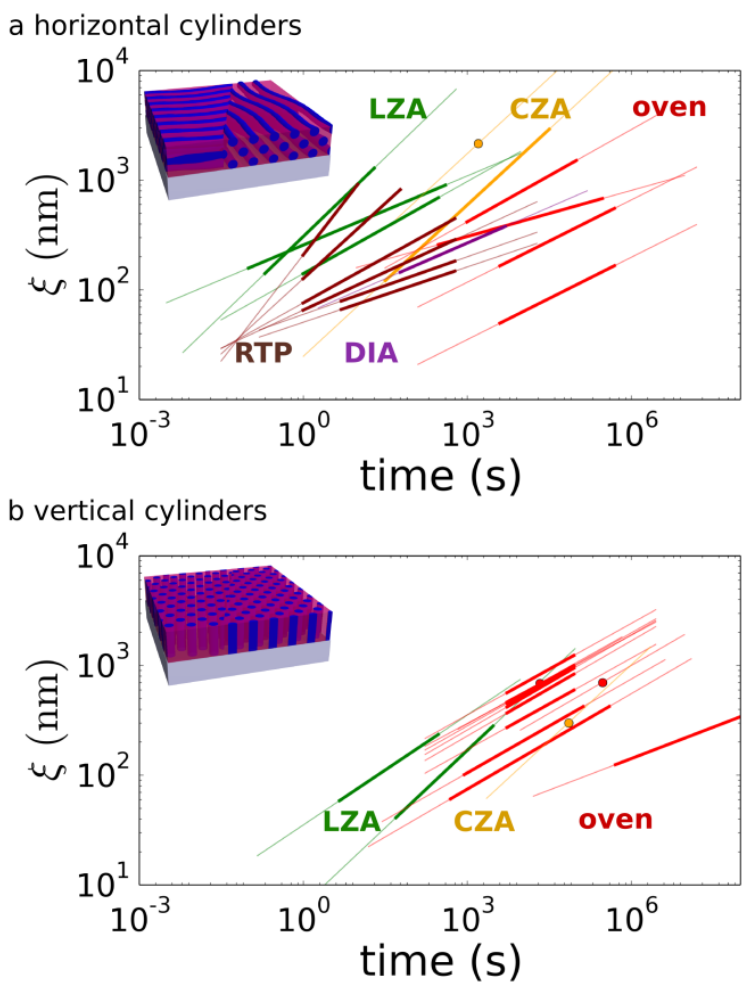

Figure 4: Grain growth curves for (a) horizontal cylinders, and (b) vertical cylinders. Data from a variety of literature sources are combined (thick lines are the power-law fits to the experimental data, thin lines are extrapolations) for oven-annealed samples (red) [57, 216, 219, 220, 223], rapid thermal processing (RTP) [237], direct immersion annealing (DIA) [474], cold zone annealing (CZA, orange) [56, 573], and laser zone annealing (LZA, green) [57]. The substantial spread in the curves comes from combining results for different materials (molecular weights) and film thicknesses; nevertheless, it is clear that dynamic annealing methods can yield improved grain sizes compared to oven annealing. 


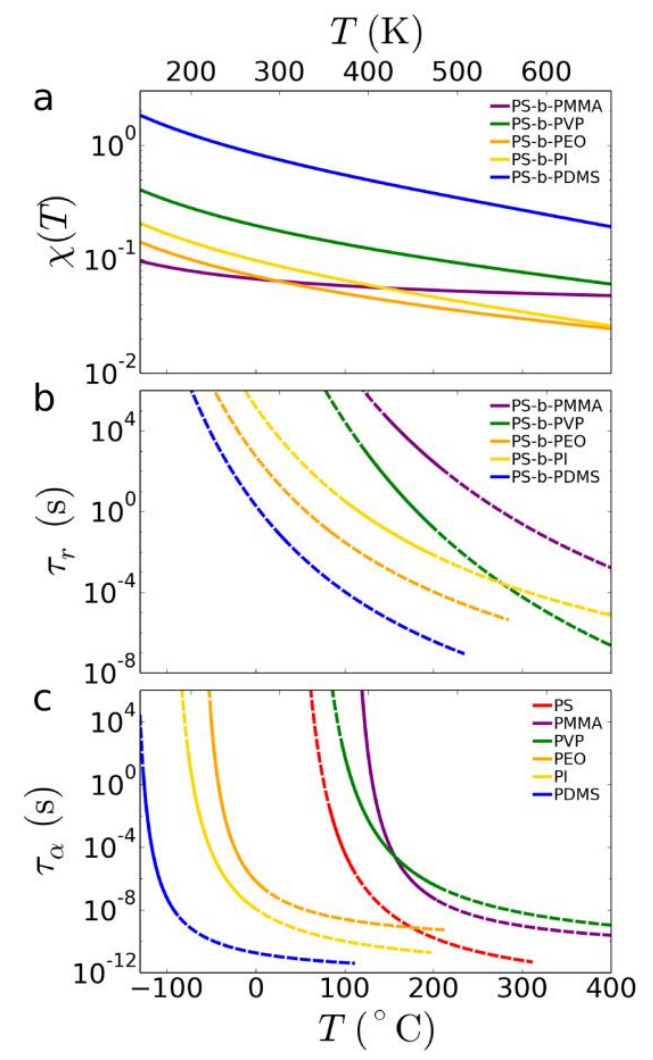

Figure 5: Intrinsic polymer and copolymer properties influence ordering timescales, including the temperaturedependence of these properties. (a) For BCPs, the segregation strength $(\chi)$ influences the sharpness of interfaces, and kinetics of assembly. A large $\chi$ promotes rapid phase separation, but hinders reordering. Annealing at higher temperature (smaller $\chi$ ) instead allows larger fluctuations and more rapid assembly. (b) The fluctuation relaxation time of the morphology is estimated using: [585, 594] $\tau_{r}=\lambda^{2} / D_{0}$, where $\lambda$ is a characteristic lengthscale (30 nm used in figure as a representative value) and $D_{0}$ is an estimate of the polymer diffusion coefficient (PS- $b$-PMMA [595], PS- $b$-P2VP [596, 597], PS- $b$-PEO [598], PS- $b$-PI [248], PS- $b$-PDMS [598]). The BCP dynamics increase rapidly as temperature is increased (dashed lines represent extrapolations assuming $D \sim D_{0} \exp (-\alpha \chi N)$ where $\alpha$ is a constant). For ordering to occur, there must be match between the timescale of BCP response, and the selected driving forces. (c) The intrinsic polymer segmental dynamics (relaxation times, $\tau_{\alpha}$ ) vary substantially for different polymer materials. Curves are based on literature values (PS [599], PMMA [600], P2VP [601], PEO [602], PI [603, 604], and PDMS [605]); dashed lines are extrapolations using the Vogel-Fulcher equation [606-609]. 

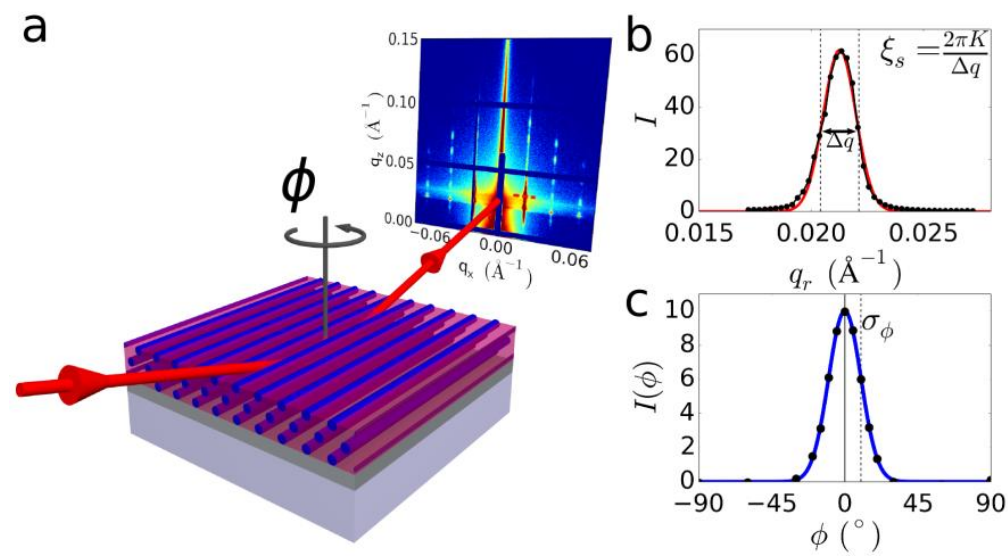

Figure 6: (a) Grazing-incidence small-angle x-ray scattering (GISAXS) can be used to measure the nanoscale structure in thin films. An x-ray beam is reflected off of substrate-supported sample at shallow angle. The peaks observed in the two-dimensional scattering image encode detailed information about structure order, including morphology type, nanostructure orientation, and average grain size. (b) A linecut through an x-ray area detector image (red dashed line) can be used to analyze the shape and width of a scattering peak. The width $(\Delta q)$ can be converted into an estimate of the grain size $\left(\xi_{s}\right)$. (c) Monitoring the intensity of a scattering peak as a function of the in-plane sample rotation angle $(\phi)$ can be used to quantify the angular spread of the morphology $\left(\sigma_{\phi}\right)$. For anisotropic samples, this allows the degree of alignment to be assessed. 

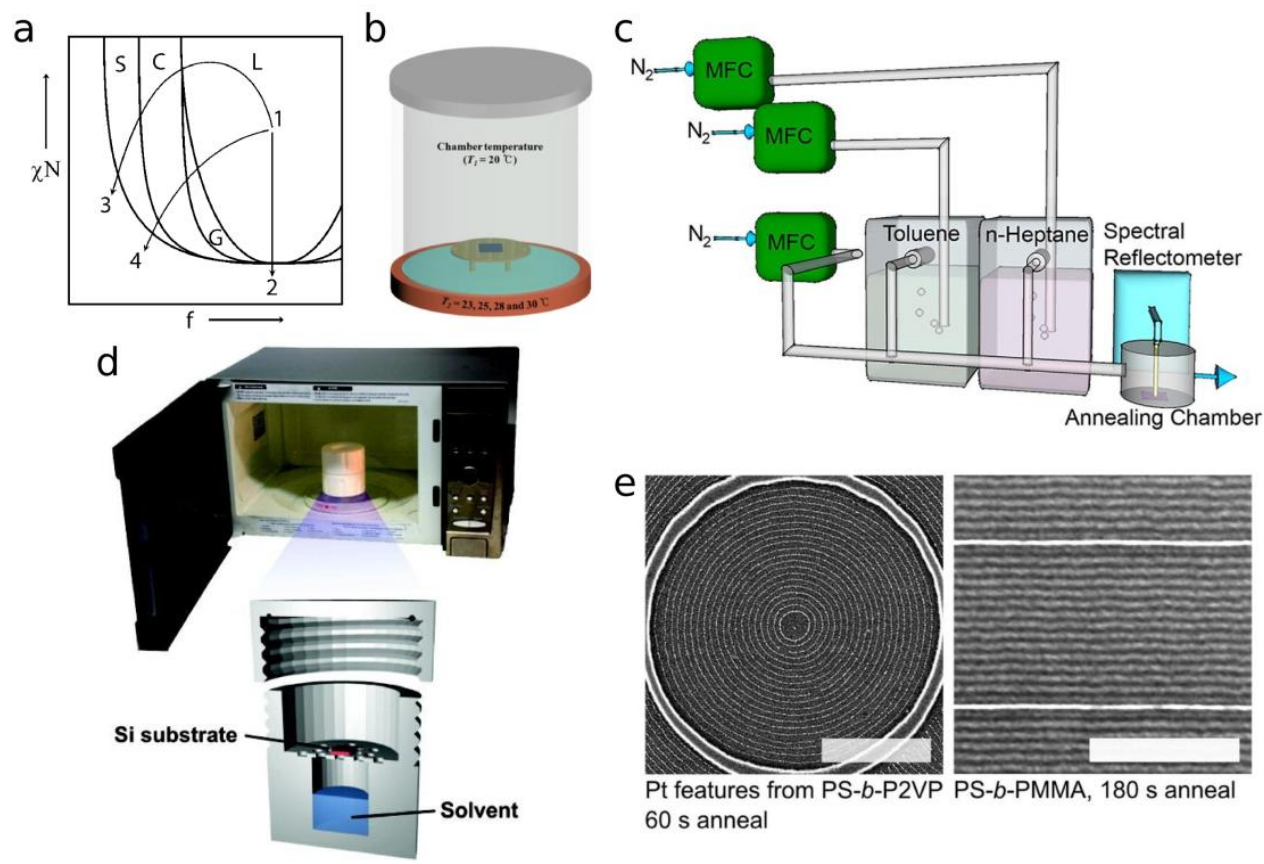
$60 \mathrm{~s}$ anneal

Figure 7: (a) SVA can be understood in terms of shifting the BCP material through its phase diagram. The arrows represent different swelling pathways ( $1-2$ non-selective solvent, $1-4$ partially selective, $1-3$ highly selective) [439]. Reproduced with permission from the American Chemical Society 2008. (b) Typical experimental setup for chamber-based ('jar') SVA processing. The sample is exposed to solvent vapors within a sealed vessel; concomitant control of chamber temperatures provides additional control [452]. Reproduced with permission from the American Chemical Society 2016. (c) Example of a flow-through SVA processing setup. Mass flow controllers (MFC) are used to control the composition of the atmosphere used for annealing [456]. Film swelling can be tracked in-situ using interferometry. Reproduced with permission from the American Chemical Society 2012. (d) Example of microwave SVA setup, wherein a sealed Teflon vessel, including solvent reservoir, is heated inside a commercial microwave oven [468]. Reproduced with permission from the American Chemical Society 2011. (e) SEMs of BCP materials that were microwave annealed under SVA conditions, on substrates with topographic features (scale bars $500 \mathrm{~nm}$ ) [117]. The left image shows a ring-shaped substrate feature, while the right shows the influence of two parallel topographic lines. The combination of microwave heating, solvent exposure, and topographic features, provides strong enforcement of structural order. Reproduced with permission from the American Chemical Society 2010. 
a

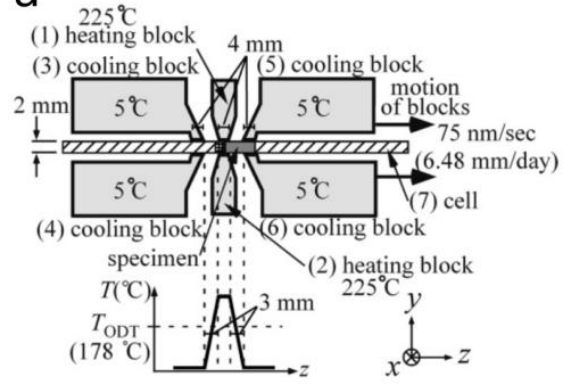

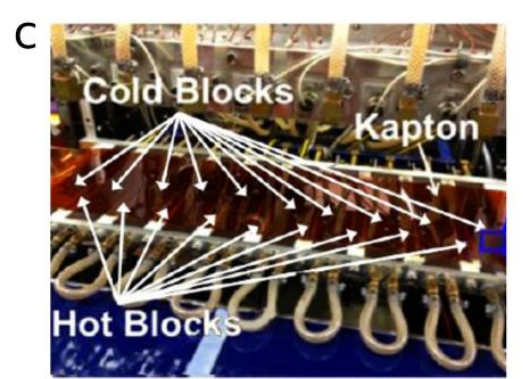
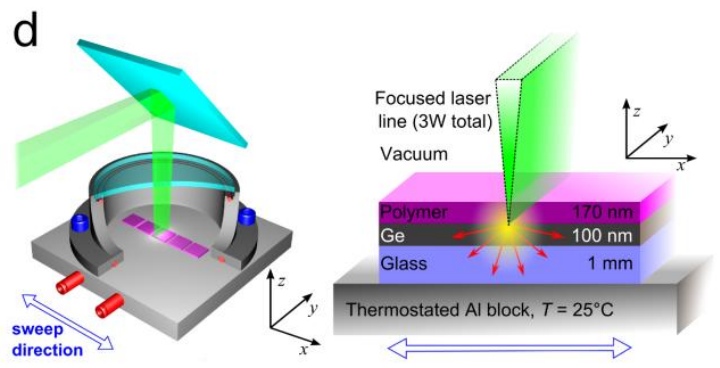

Figure 8: Experimental designs for zone annealing of block copolymers. (a) The hot zone annealing (HZA) setup described by Hashimoto et al. translates a thin sample chamber (containing a bulk BCP material) through a hot-zone defined by heating blocks [562]. Reproduced with permission from the American Chemical Society 2007. (b) Karim et al. developed a 'cold' zone annealing (CZA) setup for translating BCP thin films (cast on rigid substrates) across a thermal zone defined by thermostated blocks [56]. Reproduced with permission from the American Chemical Society 2007. (c) This approach was elaborated to enable roll-to-roll processing of films on flexible substrates [579]. Reproduced with permission from the American Chemical Society 2013. (d) Majewski and Yager developed a laser zone annealer (LZA) which enables zone annealing of thin films using larger thermal gradients [57]. Reproduced with permission from the American Chemical Society 2015. 



C
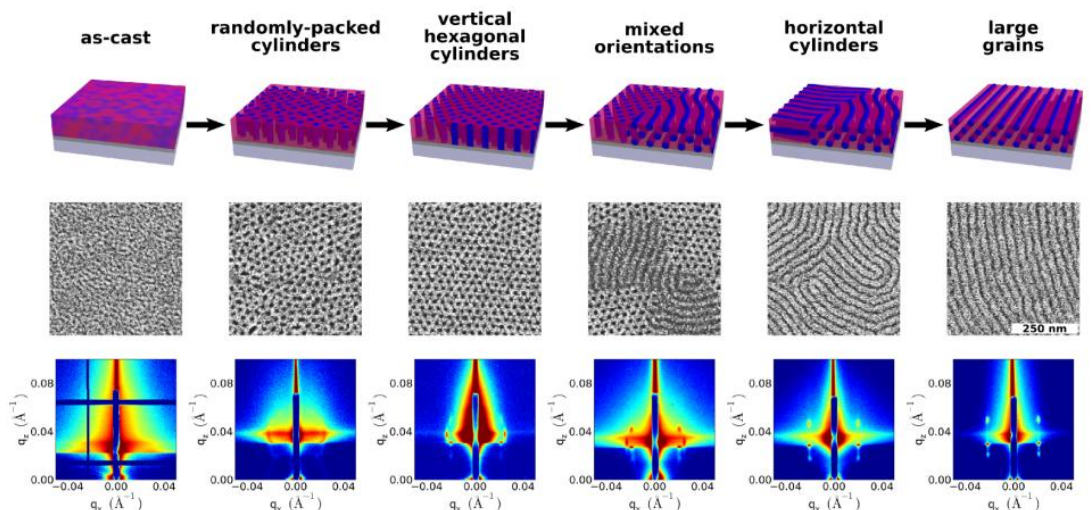

Figure 9: Examples of pathway-dependent ordering of BCPs. (a) Energy landscape for oven vs. shear ordering [211]. Reproduced with permission from the American Chemical Society 2015. Oven annealing from a disordered as-cast state induces rapid phase separation, and subsequent slow grain coarsening of an ordered state. Rapid shear can instead first break film symmetry, introducing long-range correlations (large $\xi$ ). Subsequent thermal annealing causes this 'latent alignment' state to spontaneously develop into a well-aligned and thus highly-ordered BCP films. (b) Simulation of corner-induced heterogeneous nucleation for a block-copolymer/homopolymer blend, where the coherence between nucleation points drives towards a defect-free final state [593]. Reproduced with permission from the American Chemical Society 2015. (c) Examples of transient states observed during thermal annealing of a $\mathrm{BCP}$ cylinder phase [238]. Although the final (equilibrium) state for the given thin film thickness is horizontallyordered cylinders, one observes other states at earlier ordering times, including randomly-packed cylinders, and vertically-oriented cylinders. Reproduced with permission from the Royal Society of Chemistry 2016. 


\section{Acknowledgments}

This work took place at the Center for Functional Nanomaterials, which is a U.S. DOE Office of Science Facility, at Brookhaven National Laboratory under Contract No. DE-SC0012704.

\section{References}

[1] Philp D and Stoddart J F 1996 Self-Assembly in Natural and Unnatural Systems Angewandte Chemie International Edition in English 35 1154-96

[2] Mattia E and Otto S 2015 Supramolecular systems chemistry Nat Nano 10 111-9

[3] Bates F S and Fredrickson G H 1990 Block Copolymer Thermodynamics: Theory and Experiment Annu. Rev. Phys. Chem. 41 525-57

[4] Bates F S and Fredrickson G H 1999 Block Copolymers-Designer Soft Materials Physics Today 32

[5] Fasolka M J and Mayes A M 2001 Block Copolymer Thin Films: Physics and Applications Annu. Rev. Mater. Res. 31 323-55

[6] Albert J N L and Epps T H 2010 Self-assembly of block copolymer thin films Mater. today 13 24-33

[7] Mai Y and Eisenberg A 2012 Self-assembly of block copolymers Chem. Soc. Rev. 41 5969-85

[8] Bates F S 1991 Polymer-Polymer Phase Behavior Science 251 898-905

[9] Leibler L 1980 Theory of Microphase Separation in Block Copolymers Macromol. 13 1602-17

[10] Matsen M W and Bates F S 1996 Unifying Weak- and Strong-Segregation Block Copolymer Theories Macromol. 29 1091-8

[11] Castelletto V and Hamley I W 2004 Morphologies of block copolymer melts Current Opinion in Solid State and Materials Science 8 426-38

[12] Bates F S, Schulz M F, Khandpur A K, Forster S, Rosedale J H, Almdal K and Mortensen K 1994 Fluctuations, conformational asymmetry and block copolymer phase behaviour Faraday Discuss. 98 7-18

[13] Kim G and Libera M 1998 Morphological Development in Solvent-Cast Polystyrene-Polybutadiene-Polystyrene (SBS) Triblock Copolymer Thin Films Macromol. 31 2569-77

[14] Matsen M W and Thompson R B 1999 Equilibrium behavior of symmetric ABA triblock copolymer melts The Journal of Chemical Physics 111 7139-46

[15] Knoll A, Horvat A, Lyakhova K S, Krausch G, Sevink G J A, Zvelindovsky A V and Magerle R 2002 Phase Behavior in Thin Films of Cylinder-Forming Block Copolymers Phys. Rev. Lett. 89035501

[16] Pochan D J, Chen Z, Cui H, Hales K, Qi K and Wooley K L 2004 Toroidal Triblock Copolymer Assemblies Science 306 94-7

[17] Wang C, Lee D H, Hexemer A, Kim M I, Zhao W, Hasegawa H, Ade H and Russell T P 2011 Defining the Nanostructured Morphology of Triblock Copolymers Using Resonant Soft X-ray Scattering Nano Lett. 11 3906-11

[18] Li S, Jiang Y and Chen J Z Y 2013 Morphologies and phase diagrams of ABC star triblock copolymers confined in a spherical cavity Soft Matter 9 4843-54 
[19] She M-S, Lo T-Y, Hsueh H-Y and Ho R-M 2013 Nanostructured thin films of degradable block copolymers and their applications NPG Asia Mater. 5 e42

[20] Yu B, Deng J, Li B and Shi A-C 2014 Patchy nanoparticles self-assembled from linear triblock copolymers under spherical confinement: a simulated annealing study Soft Matter 10 6831-43

[21] Mayes A M and Olvera de la Cruz M 1989 Microphase separation in multiblock copolymer melts The Journal of Chemical Physics 91 7228-35

[22] Nap R, Sushko N, Erukhimovich I and ten Brinke G 2006 Double Periodic Lamellar-inLamellar Structure in Multiblock Copolymer Melts with Competing Length Scales Macromol. 39 6765-70

[23] Kim H-C, Park S-M and Hinsberg W D 2009 Block Copolymer Based Nanostructures: Materials, Processes, and Applications to Electronics Chem. Rev. 110 146-77

[24] Huang K and Rzayev J 2009 Well-Defined Organic Nanotubes from Multicomponent Bottlebrush Copolymers J. Am. Chem. Soc. 131 6880-5

[25] Li X, Prukop S L, Biswal S L and Verduzco R 2012 Surface Properties of Bottlebrush Polymer Thin Films Macromol. 45 7118-27

[26] Verduzco R, Li X, Pesek S L and Stein G E 2015 Structure, function, self-assembly, and applications of bottlebrush copolymers Chem. Soc. Rev. 44 2405-20

[27] Mitra I, Li X, Pesek S L, Makarenko B, Lokitz B S, Uhrig D, Ankner J F, Verduzco R and Stein G E 2014 Thin Film Phase Behavior of Bottlebrush/Linear Polymer Blends Macromol. 47 5269-76

[28] Tang C, Lennon E M, Fredrickson G H, Kramer E J and Hawker C J 2008 Evolution of Block Copolymer Lithography to Highly Ordered Square Arrays Science 322 429-32

[29] Sing C E, Zwanikken J W and Olvera de la Cruz M 2014 Electrostatic control of block copolymer morphology Nat. Mater. 13 694-8

[30] Jenekhe S A and Chen X L 1999 Self-Assembly of Ordered Microporous Materials from Rod-Coil Block Copolymers Science 283 372-5

[31] Lee M, Cho B-K and Zin W-C 2001 Supramolecular Structures from Rod-Coil Block Copolymers Chem. Rev. $1013869-92$

[32] Olsen B D and Segalman R A 2008 Self-assembly of rod-coil block copolymers Mater. Sci. Eng., R 62 37-66

[33] Liu C-L, Lin C-H, Kuo C-C, Lin S-T and Chen W-C 2011 Conjugated rod-coil block copolymers: Synthesis, morphology, photophysical properties, and stimuli-responsive applications Prog. Polym. Sci. 36 603-37

[34] Verduzco R, Botiz I, Pickel D L, Kilbey S M, Hong K, Dimasi E and Darling S B 2011 Polythiophene-block-polyfluorene and Polythiophene-block-poly(fluorene-cobenzothiadiazole): Insights into the Self-Assembly of All-Conjugated Block Copolymers Macromol. 44 530-9

[35] Robb M J, Ku S-Y and Hawker C J 2013 25th Anniversary Article: No Assembly Required: Recent Advances in Fully Conjugated Block Copolymers Adv. Mater. 25 5686-700

[36] He X, Song M, Liang H and Pan C 2001 Self-assembly of the symmetric diblock copolymer in a confined state: Monte Carlo simulation The Journal of Chemical Physics 114 10510-3 
[37] Wu Y, Cheng G, Katsov K, Sides S W, Wang J, Tang J, Fredrickson G H, Moskovits M and Stucky G D 2004 Composite mesostructures by nano-confinement Nat. Mater. 3 81622

[38] Yu B, Sun P, Chen T, Jin Q, Ding D, Li B and Shi A-C 2006 Confinement-Induced Novel Morphologies of Block Copolymers Phys. Rev. Lett. 96138306

[39] Jung Y S, Jung W and Ross C A 2008 Nanofabricated Concentric Ring Structures by Templated Self-Assembly of a Diblock Copolymer Nano Lett. 8 2975-81

[40] Bang J, Jeong U, Ryu D Y, Russell T P and Hawker C J 2009 Block Copolymer Nanolithography: Translation of Molecular Level Control to Nanoscale Patterns $A d v$. Mater. 21 4769-92

[41] Chi P, Wang Z, Li B and Shi A-C 2011 Soft Confinement-Induced Morphologies of Diblock Copolymers Langmuir 27 11683-9

[42] Simao C, Francone A, Borah D, Lorret O, Salaun M, Kosmala B, Shaw M T, Dittert B, Kehagias N, Zelsmann M, Morris M A and Torres C M S 2012 Soft Graphoepitaxy of Hexagonal PS-b-PDMS on Nanopatterned POSS Surfaces fabricated by Nanoimprint Lithography J. Photopolym. Sci. Technol. 25 239-44

[43] Jin Z and Fan H 2014 Self-assembly of nanostructured block copolymer nanoparticles Soft Matter 10 9212-9

[44] Yabu H, Higuchi T and Jinnai H 2014 Frustrated phases: polymeric self-assemblies in a 3D confinement Soft Matter 10 2919-31

[45] Fasolka M J, Banerjee P, Mayes A M, Pickett G and Balazs A C 2000 Morphology of Ultrathin Supported Diblock Copolymer Films: Theory and Experiment Macromol. 33 $5702-12$

[46] Segalman R A 2005 Patterning with block copolymer thin films Mater. Sci. Eng., $R \mathbf{4 8}$ $191-226$

[47] Stein G E, Kramer E J, Li X and Wang J 2007 Layering Transitions in Thin Films of Spherical-Domain Block Copolymers Macromol. 40 2453-60

[48] Darling S B 2007 Directing the self-assembly of block copolymers Prog. Polym. Sci. 32 1152-204

[49] Hamley I W 2009 Ordering in thin films of block copolymers: Fundamentals to potential applications Prog. Polym. Sci. 34 1161-210

[50] Hu H, Gopinadhan M and Osuji C O 2014 Directed self-assembly of block copolymers: a tutorial review of strategies for enabling nanotechnology with soft matter Soft Matter 10 3867-89

[51] Hashimoto T, Shibayama M and Kawai H 1983 Ordered structure in block polymer solutions. 4. Scaling rules on size of fluctuations with block molecular weight, concentration, and temperature in segregation and homogeneous regimes Macromol. 16 1093-101

[52] Mansky P, Tsui O K C, Russell T P and Gallot Y 1999 Phase Coherence and Microphase Separation Transitions in Diblock Copolymer Thin Films Macromol. 32 4832-7

[53] Yager K G, Forrey C, Singh G, Satija S, Page K, Patton D, Douglas J, Jones R and Karim A 2015 Thermally-Induced Transition of Lamellae Orientation in Block-Copolymer Films on 'Neutral' Nanoparticle-Coated Substrates Soft Matter 11 5154-67

[54] Albert J N L, Bogart T D, Lewis R L, Beers K L, Fasolka M J, Hutchison J B, Vogt B D and Epps T H 2011 Gradient Solvent Vapor Annealing of Block Copolymer Thin Films Using a Microfluidic Mixing Device Nano Lett. 11 1351-7 
[55] Hannon A F, Bai W, Alexander-Katz A and Ross C A 2015 Simulation methods for solvent vapor annealing of block copolymer thin films Soft Matter 11 3794-805

[56] Berry B C, Bosse A W, Douglas J F, Jones R L and Karim A 2007 Orientational Order in Block Copolymer Films Zone Annealed below the Order-Disorder Transition Temperature Nano Lett. 7 2789-94

[57] Majewski P W and Yager K G 2015 Millisecond Ordering of Block Copolymer Films via Photothermal Gradients ACS Nano 9 3896-906

[58] Koppi K A, Tirrell M and Bates F S 1993 Shear-induced isotropic-to-lamellar transition Phys. Rev. Lett. 701449

[59] Riise B L, Fredrickson G H, Larson R G and Pearson D S 1995 Rheology and ShearInduced Alignment of Lamellar Diblock and Triblock Copolymers Macromol. 28 7653-9

[60] Angelescu D E, Waller J H, Adamson D H, Deshpande P, Chou S Y, Register R A and Chaikin P M 2004 Macroscopic Orientation of Block Copolymer Cylinders in SingleLayer Films by Shearing Adv. Mater. 16 1736-40

[61] Rychkov I 2005 Block Copolymers Under Shear Flow Macromol. Theory Simul. 14 20742

[62] Hong Y-R, Adamson D H, Chaikin P M and Register R A 2009 Shear-induced sphere-tocylinder transition in diblock copolymer thin films Soft Matter 5 1687-91

[63] Nikoubashman A, Register R A and Panagiotopoulos A Z 2013 Simulations of shearinduced morphological transitions in block copolymers Soft Matter 9 9960-71

[64] Pereira G G and Williams D R M 1999 Diblock Copolymer Melts in Electric Fields: The Transition from Parallel to Perpendicular Alignment Using a Capacitor Analogy Macromol. 32 8115-20

[65] Ashok B, Muthukumar M and Russell T P 2001 Confined thin film diblock copolymer in the presence of an electric field J. Chem. Phys. 115 1559-64

[66] Tsori Y and Andelman D 2002 Thin Film Diblock Copolymers in Electric Field: Transition from Perpendicular to Parallel Lamellae Macromol. 35 5161-70

[67] Liedel C, Pester C W, Ruppel M, Urban V S and Böker A 2012 Beyond Orientation: The Impact of Electric Fields on Block Copolymers Macromol. Chem. Phys. 213 259-69

[68] Osuji C, Ferreira P J, Mao G, Ober C K, Vander Sande J B and Thomas E L 2004 Alignment of Self-Assembled Hierarchical Microstructure in Liquid Crystalline Diblock Copolymers Using High Magnetic Fields Macromol. 37 9903-8

[69] Majewski P W, Gopinadhan M, Jang W-S, Lutkenhaus J L and Osuji C O 2010 Anisotropic Ionic Conductivity in Block Copolymer Membranes by Magnetic Field Alignment J. Am. Chem. Soc. 132 17516-22

[70] Gopinadhan M, Majewski P W and Osuji C O 2010 Facile Alignment of Amorphous Poly(ethylene oxide) Microdomains in a Liquid Crystalline Block Copolymer Using Magnetic Fields: Toward Ordered Electrolyte Membranes Macromol. 43 3286-93

[71] Majewski P W, Gopinadhan M and Osuji C O 2012 Magnetic field alignment of block copolymers and polymer nanocomposites: Scalable microstructure control in functional soft materials J. Polym. Sci., Part B: Polym. Phys. 50 2-8

[72] McCulloch B, Portale G, Bras W, Pople J A, Hexemer A and Segalman R A 2013 Dynamics of Magnetic Alignment in Rod-Coil Block Copolymers Macromol. $464462-$ 71

[73] Cheng J Y, Ross C A, Smith H I and Thomas E L 2006 Templated self-assembly of block copolymers: Top-down helps bottom-up Adv. Mater. 18 2505-21 
[74] Koo K, Ahn H, Kim S-W, Ryu D Y and Russell T P 2013 Directed self-assembly of block copolymers in the extreme: guiding microdomains from the small to the large Soft Matter 9 9059-71

[75] Stein G E, Mahadevapuram N and Mitra I 2014 Controlling interfacial interactions for directed self assembly of block copolymers J. Polym. Sci., Part B: Polym. Phys. 53 96102

[76] Thompson R B, Ginzburg V V, Matsen M W and Balazs A C 2001 Predicting the Mesophases of Copolymer-Nanoparticle Composites Science 292 2469-72

[77] Chiu J J, Kim B J, Kramer E J and Pine D J 2005 Control of Nanoparticle Location in Block Copolymers J. Am. Chem. Soc. 127 5036-7

[78] Lin Y, Boker A, He J, Sill K, Xiang H, Abetz C, Li X, Wang J, Emrick T, Long S, Wang Q, Balazs A and Russell T P 2005 Self-directed self-assembly of nanoparticle/copolymer mixtures Nature $\mathbf{4 3 4} 55$

[79] Haryono A and Binder W H 2006 Controlled Arrangement of Nanoparticle Arrays in Block-Copolymer Domains Small 2 600-11

[80] Kim B J, Bang J, Hawker C J and Kramer E J 2006 Effect of Areal Chain Density on the Location of Polymer-Modified Gold Nanoparticles in a Block Copolymer Template Macromol. 39 4108-14

[81] Chiu J J, Kim B J, Yi G-R, Bang J, Kramer E J and Pine D J 2007 Distribution of Nanoparticles in Lamellar Domains of Block Copolymers Macromol. 40 3361-5

[82] Löwenhaupt B and Hellmann G P 1991 Microphase and macrophase separation in blends with a two-block copolymer Polymer 32 1065-76

[83] Orso K A and Green P F 1999 Phase Behavior of Thin Film Blends of Block Copolymers and Homopolymers: Changes in Domain Dimensions Macromol. 32 1087-92

[84] Jeong U, Ryu D Y, Kho D H, Lee D H, Kim J K and Russell T P 2003 Phase Behavior of Mixtures of Block Copolymer and Homopolymers in Thin Films and Bulk Macromol. 36 3626-34

[85] Mykhaylyk T A, Collins S, Hamley I W, Evans S D and Henderson J R 2004 Ordered structures and phase transitions in thin films of polystyrene/polyisoprene block copolymer and blends with the corresponding homopolymers J. Mater. Sci. 39 2249-52

[86] Peng J, Gao X, Wei Y, Wang H, Li B and Han Y 2005 Controlling the size of nanostructures in thin films via blending of block copolymers and homopolymers The Journal of Chemical Physics 122114706

[87] Hadziioannou G and Skoulios A 1982 Structural study of mixtures of styrene isoprene two- and three-block copolymers Macromol. 15 267-71

[88] Hashimoto T, Koizumi S and Hasegawa H 1994 Ordered Structure in Blends of Block Copolymers.2.Self-Assembly for Immiscible Lamella-Forming Copolymers Macromol. 27 1562-70

[89] Koizumi S, Hasegawa H and Hashimoto T 1994 Ordered Structure in Blends of Block Copolymers. 3. Self-Assembly in Blends of Sphere- or Cylinder-Forming Copolymers Macromol. 27 4371-81

[90] Matsen M W 1995 Immiscibility of large and small symmetric diblock copolymers The Journal of Chemical Physics 103 3268-71

[91] Matsen M W and Bates F S 1995 One-Component Approximation for Binary Diblock Copolymer Blends Macromol. 28 7298-300 
[92] Spontak R J, Fung J C, Braunfeld M B, Sedat J W, Agard D A, Kane L, Smith S D, Satkowski M M, Ashraf A, Hajduk D A and Gruner S M 1996 Phase Behavior of Ordered Diblock Copolymer Blends: Effect of Compositional Heterogeneity Macromol. 29 4494-507

[93] Zhao J, Majumdar B, Schulz M F, Bates F S, Almdal K, Mortensen K, Hajduk D A and Gruner S M 1996 Phase Behavior of Pure Diblocks and Binary Diblock Blends of Poly(ethylene)-Poly(ethylethylene) Macromol. 29 1204-15

[94] Abetz V and Goldacker T 2000 Formation of superlattices via blending of block copolymers Macromol. Rapid Commun. 21 16-34

[95] Yager K G, Lai E and Black C T 2014 Self-Assembled Phases of Block Copolymer Blend Thin Films ACS Nano 8 10582-8

[96] Williamson L D and Nealey P F 2015 Macrophase Separation of Blends of Diblock Copolymers in Thin Films Macromol. 48 3997-4003

[97] Ruzette A-V and Leibler L 2005 Block copolymers in tomorrow's plastics Nat. Mater. 4 19-31

[98] Kim J K, Yang S Y, Lee Y and Kim Y 2010 Functional nanomaterials based on block copolymer self-assembly Prog. Polym. Sci. 35 1325-49

[99] Bai W and Ross C A 2016 Functional nanostructured materials based on self-assembly of block copolymers Mrs Bull 41 100-7

[100] Li M and Ober C K 2006 Block copolymer patterns and templates Mater. today 930

[101] Kim H-C and Hinsberg W D 2008 Surface patterns from block copolymer self-assembly J. Vac. Sci. Technol., A 26 1369-82

[102] Luo M and Epps T H 2013 Directed Block Copolymer Thin Film Self-Assembly: Emerging Trends in Nanopattern Fabrication Macromol. 46 7567-79

[103] Jeong S-J, Kim J Y, Kim B H, Moon H-S and Kim S O 2013 Directed self-assembly of block copolymers for next generation nanolithography Mater. today 16 468-76

[104] Kun-Hua T, Wubin B, George L, Konstantinos N, Apostolos A and Caroline A R 2015 Universal pattern transfer methods for metal nanostructures by block copolymer lithography Nanotechnology 26375301

[105] Schwartz E L and Ober C K 2010 Advanced Nanomaterials: Wiley-VCH Verlag GmbH \& Co. KGaA) pp 1-66

[106] Nunns A, Gwyther J and Manners I 2013 Inorganic block copolymer lithography Polymer 54 1269-84

[107] Cummins C, Ghoshal T, Holmes J D and Morris M A 2016 Strategies for Inorganic Incorporation using Neat Block Copolymer Thin Films for Etch Mask Function and Nanotechnological Application Adv. Mater. n/a-n/a

[108] Thurn-Albrecht T, Steiner R, DeRouchey J, Stafford C M, Huang E, Bal M, Tuominen M, Hawker C J and Russell T P 2000 Nanoscopic Templates from Oriented Block Copolymer Films Adv. Mater. 12 787-91

[109] Hillmyer M 2005 Block Copolymers II, ed V Abetz: Springer Berlin Heidelberg) pp 13781

[110] Jung Y S and Ross C A 2009 Well-Ordered Thin-Film Nanopore Arrays Formed Using a Block-Copolymer Template Small 5 1654-9

[111] Jung Y S, Lee J H, Lee J Y and Ross C A 2010 Fabrication of Diverse Metallic Nanowire Arrays Based on Block Copolymer Self-Assembly Nano Lett. 10 3722-6 
[112] Kim S Y, Gwyther J, Manners I, Chaikin P M and Register R A 2014 Metal-Containing Block Copolymer Thin Films Yield Wire Grid Polarizers with High Aspect Ratio Adv. Mater. 26 791-5

[113] Lopes W A and Jaeger H M 2001 Hierarchical self-assembly of metal nanostructures on diblock copolymer scaffolds Nature $\mathbf{4 1 4}$ 735-8

[114] Warren S C, Messina L C, Slaughter L S, Kamperman M, Zhou Q, Gruner S M, DiSalvo F J and Wiesner U 2008 Ordered Mesoporous Materials from Metal Nanoparticle-Block Copolymer Self-Assembly Science 320 1748-52

[115] Chai J, Wang D, Fan X and Buriak J M 2007 Assembly of aligned linear metallic patterns on silicon Nat. Nanotechnol. 2 500-6

[116] Chai J and Buriak J M 2008 Using Cylindrical Domains of Block Copolymers To SelfAssemble and Align Metallic Nanowires ACS Nano 2 489-501

[117] Zhang X, Harris K D, Wu N L Y, Murphy J N and Buriak J M 2010 Fast Assembly of Ordered Block Copolymer Nanostructures through Microwave Annealing ACS Nano 4 7021-9

[118] Cha S K, Mun J H, Chang T, Kim S Y, Kim J Y, Jin H M, Lee J Y, Shin J, Kim K H and Kim S O $2015 \mathrm{Au}-$ Ag Core-Shell Nanoparticle Array by Block Copolymer Lithography for Synergistic Broadband Plasmonic Properties ACS Nano 9 5536-43

[119] Majewski P W, Rahman A, Black C T and Yager K G 2015 Arbitrary lattice symmetries via block copolymer nanomeshes Nat. Commun. 67448

[120] Kowalewski T, Tsarevsky N V and Matyjaszewski K 2002 Nanostructured Carbon Arrays from Block Copolymers of Polyacrylonitrile J. Am. Chem. Soc. 124 10632-3

[121] Kosonen H, Valkama S, Nykänen A, Toivanen M, ten Brinke G, Ruokolainen J and Ikkala O 2006 Functional Porous Structures Based on the Pyrolysis of Cured Templates of Block Copolymer and Phenolic Resin Adv. Mater. 18 201-5

[122] Valkama S, Nykänen A, Kosonen H, Ramani R, Tuomisto F, Engelhardt P, ten Brinke G, Ikkala O and Ruokolainen J 2007 Hierarchical Porosity in Self-Assembled Polymers: Post-Modification of Block Copolymer-Phenolic Resin Complexes by Pyrolysis Allows the Control of Micro- and Mesoporosity Advanced Functional Materials 17 183-90

[123] Xue J, Singh G, Qiang Z, Yager K G, Karim A and Vogt B D 2013 Facile control of long range orientation in mesoporous carbon films with thermal zone annealing velocity Nanoscale 5 12440-7

[124] Zhao D, Feng J, Huo Q, Melosh N, Fredrickson G H, Chmelka B F and Stucky G D 1998 Triblock Copolymer Syntheses of Mesoporous Silica with Periodic 50 to 300 Angstrom Pores Science 279 548-52

[125] Melosh N A, Lipic P, Bates F S, Wudl F, Stucky G D, Fredrickson G H and Chmelka B F 1999 Molecular and Mesoscopic Structures of Transparent Block Copolymer-Silica Monoliths Macromol. 32 4332-42

[126] Bhaway S, Kisslinger K, Zhang L, Yager K G, Schmitt A L, Mahanthappa M K, Karim A and Vogt B D 2014 Mesoporous Carbon-Vanadium Oxide Films by Resol-Assisted, Triblock Copolymer-Templated Cooperative Self-Assembly ACS Applied Materials \& Interfaces

[127] Schüth F 2001 Non-siliceous Mesostructured and Mesoporous Materials Chem. Mater. 13 3184-95

[128] Deng Y, Wei J, Sun Z and Zhao D 2013 Large-pore ordered mesoporous materials templated from non-Pluronic amphiphilic block copolymers Chem. Soc. Rev. 42 4054-70 
[129] Peng Q, Tseng Y-C, Darling S B and Elam J W 2010 Nanoscopic Patterned Materials with Tunable Dimensions via Atomic Layer Deposition on Block Copolymers $A d v$. Mater. 22 5129-33

[130] Peng Q, Tseng Y-C, Darling S B and Elam J W 2011 A Route to Nanoscopic Materials via Sequential Infiltration Synthesis on Block Copolymer Templates ACS Nano 5 4600-6

[131] Tseng Y-C, Peng Q, Ocola L E, Czaplewski D A, Elam J W and Darling S B 2011 Etch properties of resists modified by sequential infiltration synthesis Journal of Vacuum Science \&amp; Technology B 29 -

[132] Tseng Y-C, Peng Q, Ocola L E, Elam J W and Darling S B 2011 Enhanced Block Copolymer Lithography Using Sequential Infiltration Synthesis The Journal of Physical Chemistry C 115 17725-9

[133] Kamcev J, Germack D S, Nykypanchuk D, Grubbs R B, Nam C-Y and Black C T 2012 Chemically Enhancing Block Copolymers for Block-Selective Synthesis of SelfAssembled Metal Oxide Nanostructures ACS Nano 7 339-46

[134] Ramanathan M, Tseng Y-C, Ariga K and Darling S B 2013 Emerging trends in metalcontaining block copolymers: synthesis, self-assembly, and nanomanufacturing applications Journal of Materials Chemistry C $12080-91$

[135] Park M, Harrison C, Chaikin P M, Register R A and Adamson D H 1997 Block Copolymer Lithography: Periodic Arrays of $\sim 1011$ Holes in 1?Square Centimeter Science 276 1401-4

[136] Park M, Chaikin P M, Register R A and Adamson D H 2001 Large area dense nanoscale patterning of arbitrary surfaces Appl. Phys. Lett. 79 257-9

[137] Terris B D and Thomson T 2005 Nanofabricated and self-assembled magnetic structures as data storage media Journal of Physics D: Applied Physics 38 R199-R222

[138] Jung Y S, Jung W, Tuller H L and Ross C A 2008 Nanowire Conductive Polymer Gas Sensor Patterned Using Self-Assembled Block Copolymer Lithography Nano Lett. 8 3776-80

[139] Papalia J M, Adamson D H, Chaikin P M and Register R A 2010 Silicon nanowire polarizers for far ultraviolet (sub-200 nm) applications: Modeling and fabrication J. Appl. Phys. 107084305

[140] Bates C M, Maher M J, Janes D W, Ellison C J and Willson C G 2013 Block Copolymer Lithography Macromol. 47 2-12

[141] Kim S Y, Nunns A, Gwyther J, Davis R L, Manners I, Chaikin P M and Register R A 2014 Large-Area Nanosquare Arrays from Shear-Aligned Block Copolymer Thin Films Nano Lett. 14 5698-705

[142] Ghoshal T, Senthamaraikannan R, Shaw M T, Holmes J D and Morris M A 2014 Fabrication of Ordered, Large Scale, Horizontally-Aligned Si Nanowire Arrays Based on an In Situ Hard Mask Block Copolymer Approach Adv. Mater. 26 1207-16

[143] Sidorenko A, Tokarev I, Minko S and Stamm M 2003 Ordered Reactive Nanomembranes/Nanotemplates from Thin Films of Block Copolymer Supramolecular Assembly J. Am. Chem. Soc. 125 12211-6

[144] Thurn-Albrecht T, Schotter J, Kastle G A, Emley N, Shibauchi T, Krusin-Elbaum L, Guarini K, Black C T, Tuominen M T and Russell T P 2000 Ultrahigh-Density Nanowire Arrays Grown in Self-Assembled Diblock Copolymer Templates Science 290 2126-9

[145] Yoo H G, Byun M, Jeong C K and Lee K J 2015 Performance Enhancement of Electronic and Energy Devices via Block Copolymer Self-Assembly Adv. Mater. 27 3982-98 
[146] Tseng Y-C and Darling S B 2010 Block Copolymer Nanostructures for Technology Polymers 2 470-89

[147] Soo P P, Huang B, Jang Y I, Chiang Y M, Sadoway D R and Mayes A M 1999 Rubbery Block Copolymer Electrolytes for Solid-State Rechargeable Lithium Batteries $J$. Electrochem. Soc. 146 32-7

[148] Ruzette A-V G, Soo P P, Sadoway D R and Mayes A M 2001 Melt-Formable Block Copolymer Electrolytes for Lithium Rechargeable Batteries J. Electrochem. Soc. 148 A537-A43

[149] Singh M, Odusanya O, Wilmes G M, Eitouni H B, Gomez E D, Patel A J, Chen V L, Park M J, Fragouli P, Iatrou H, Hadjichristidis N, Cookson D and Balsara N P 2007 Effect of Molecular Weight on the Mechanical and Electrical Properties of Block Copolymer Electrolytes Macromol. 40 4578-85

[150] Panday A, Mullin S, Gomez E D, Wanakule N, Chen V L, Hexemer A, Pople J and Balsara N P 2009 Effect of Molecular Weight and Salt Concentration on Conductivity of Block Copolymer Electrolytes Macromol. 42 4632-7

[151] Ghosh A, Wang C and Kofinas P 2010 Block Copolymer Solid Battery Electrolyte with High Li-Ion Transference Number J. Electrochem. Soc. 157 A846-A9

[152] Hallinan D T, Mullin S A, Stone G M and Balsara N P 2013 Lithium Metal Stability in Batteries with Block Copolymer Electrolytes J. Electrochem. Soc. 160 A464-A70

[153] Bouchet R, Maria S, Meziane R, Aboulaich A, Lienafa L, Bonnet J-P, Phan T N T, Bertin D, Gigmes D, Devaux D, Denoyel R and Armand M 2013 Single-ion BAB triblock copolymers as highly efficient electrolytes for lithium-metal batteries Nat. Mater. 12 4527

[154] Javier A E, Patel S N, Hallinan D T, Srinivasan V and Balsara N P 2011 Simultaneous Electronic and Ionic Conduction in a Block Copolymer: Application in Lithium Battery Electrodes Angewandte Chemie International Edition 50 9848-51

[155] Choudhury S, Agrawal M, Formanek P, Jehnichen D, Fischer D, Krause B, Albrecht V, Stamm M and Ionov L 2015 Nanoporous Cathodes for High-Energy Li-S Batteries from Gyroid Block Copolymer Templates ACS Nano 9 6147-57

[156] Samant S P, Grabowski C A, Kisslinger K, Yager K G, Yuan G, Satija S K, Durstock M F, Raghavan D and Karim A 2016 Directed Self-Assembly of Block Copolymers for High Breakdown Strength Polymer Film Capacitors ACS Applied Materials \& Interfaces 8 7966-76

[157] Song I Y, Kwon Y S, Lim J and Park T 2014 Well-Defined All-Conducting Block Copolymer Bilayer Hybrid Nanostructure: Selective Positioning of Lithium Ions and Efficient Charge Collection ACS Nano 8 6893-901

[158] Crossland E J W, Kamperman M, Nedelcu M, Ducati C, Wiesner U, Smilgies D M, Toombes G E S, Hillmyer M A, Ludwigs S, Steiner U and Snaith H J 2009 A Bicontinuous Double Gyroid Hybrid Solar Cell Nano Lett. 9 2807-12

[159] Topham P D, Parnell A J and Hiorns R C 2011 Block copolymer strategies for solar cell technology J. Polym. Sci., Part B: Polym. Phys. 49 1131-56

[160] Lee Y and Gomez E D 2015 Challenges and Opportunities in the Development of Conjugated Block Copolymers for Photovoltaics Macromol. 48 7385-95

[161] Jeong C K, Baek K M, Niu S, Nam T W, Hur Y H, Park D Y, Hwang G-T, Byun M, Wang Z L, Jung Y S and Lee K J 2014 Topographically-Designed Triboelectric Nanogenerator via Block Copolymer Self-Assembly Nano Lett. 14 7031-8 
[162] Checco A, Rahman A and Black C T 2014 Robust Superhydrophobicity in Large-Area Nanostructured Surfaces Defined by Block-Copolymer Self Assembly Adv. Mater. 26 $886-91$

[163] Checco A, Ocko B M, Rahman A, Black C T, Tasinkevych M, Giacomello A and Dietrich S 2014 Collapse and Reversibility of the Superhydrophobic State on Nanotextured Surfaces Phys. Rev. Lett. 112216101

[164] Jeong C K, Jin H M, Ahn J-H, Park T J, Yoo H G, Koo M, Choi Y-K, Kim S O and Lee K J 2014 Electrical Biomolecule Detection Using Nanopatterned Silicon via Block Copolymer Lithography Small 10 337-43

[165] Fang Y, Jiao Y, Xiong K, Ogier R, Yang Z-J, Gao S, Dahlin A B and Käll M 2015 Plasmon Enhanced Internal Photoemission in Antenna-Spacer-Mirror Based Au/TiO2 Nanostructures Nano Lett. 15 4059-65

[166] Bonod N 2015 Silicon photonics: Large-scale dielectric metasurfaces Nat. Mater. 14 6645

[167] Perl E E, McMahon W E, Farrell R M, DenBaars S P, Speck J S and Bowers J E 2014 Surface Structured Optical Coatings with Near-Perfect Broadband and Wide-Angle Antireflective Properties Nano Lett. 14 5960-4

[168] Rahman A, Ashraf A, Xin H, Tong X, Sutter P, Eisaman M D and Black C T 2015 Sub50-nm self-assembled nanotextures for enhanced broadband antireflection in silicon solar cells Nat Commun $\mathbf{6}$

[169] Chen J-D, Cui C, Li Y-Q, Zhou L, Ou Q-D, Li C, Li Y and Tang J-X 2015 SingleJunction Polymer Solar Cells Exceeding 10\% Power Conversion Efficiency Adv. Mater. 27 1035-41

[170] Paetzold U W, Lehnen S, Bittkau K, Rau U and Carius R 2014 Nanoscale Observation of Waveguide Modes Enhancing the Efficiency of Solar Cells Nano Lett. 14 6599-605

[171] Chou S Y and Ding W 2013 Ultrathin, high-efficiency, broad-band, omni-acceptance, organic solar cells enhanced by plasmonic cavity with subwavelength hole array $O p t$. Express 21 A60-A76

[172] Ding W, Wang Y, Chen H and Chou S Y 2014 Plasmonic Nanocavity Organic LightEmitting Diode with Significantly Enhanced Light Extraction, Contrast, Viewing Angle, Brightness, and Low-Glare Advanced Functional Materials 24 6329-39

[173] Black C T, Guarini K W, Ruiz R, Sikorski E M, Babich I V, Sandstrom R L and Zhang Y 2006 Polymer self assembly in semiconductor microelectronics. In: Electron Devices Meeting, 2006. IEDM '06. International, pp 1-4

[174] Black C T, Ruiz R, Breyta G, Cheng J Y, Colburn M E, Guarini K W, Kim H-C and Zhang Y 2007 Polymer self assembly in semiconductor microelectronics IBM Journal of Research and Development 51605

[175] Morris M A 2015 Directed self-assembly of block copolymers for nanocircuitry fabrication Microelectron. Eng. 132 207-17

[176] Ji S, Wan L, Liu C-C and Nealey P F 2015 Directed self-assembly of block copolymers on chemical patterns: A platform for nanofabrication Prog. Polym. Sci. 54-55 76-127

[177] Li W and Müller M Directed self-assembly of block copolymers by chemical or topographical guiding patterns: Optimizing molecular architecture, thin-film properties, and kinetics Prog. Polym. Sci. 
[178] Ruiz R, Kang H, Detcheverry F A, Dobisz E, Kercher D S, Albrecht T R, de Pablo J J and Nealey P F 2008 Density Multiplication and Improved Lithography by Directed Block Copolymer Assembly Science 321 936-9

[179] Bita I, Yang J K W, Jung Y S, Ross C A, Thomas E L and Berggren K K 2008 Graphoepitaxy of Self-Assembled Block Copolymers on Two-Dimensional Periodic Patterned Templates Science 321 939-43

[180] Yang J K W, Jung Y S, Chang J-B, Mickiewicz R A, Alexander Katz A, Ross C A and Berggren K K 2010 Complex self-assembled patterns using sparse commensurate templates with locally varying motifs Nat. Nanotechnol. 5 256-60

[181] Liu C-C, Ramírez-Hernández A, Han E, Craig G S W, Tada Y, Yoshida H, Kang H, Ji S, Gopalan P, de Pablo J J and Nealey P F 2013 Chemical Patterns for Directed SelfAssembly of Lamellae-Forming Block Copolymers with Density Multiplication of Features Macromol. 46 1415-24

[182] Yang Y-B, Choi Y J, Kim S O and Kim J U 2015 Directed self-assembly of cylinderforming diblock copolymers on sparse chemical patterns Soft Matter 11 4496-506

[183] Stoykovich M P, Kang H, Daoulas K C, Liu G, Liu C-C, de Pablo J J, Müller M and Nealey P F 2007 Directed Self-Assembly of Block Copolymers for Nanolithography: Fabrication of Isolated Features and Essential Integrated Circuit Geometries ACS Nano 1 168-75

[184] Ross C A, Berggren K K, Cheng J Y, Jung Y S and Chang J-B 2014 Three-Dimensional Nanofabrication by Block Copolymer Self-Assembly Adv. Mater. 26 4386-96

[185] Chang J-B, Choi H K, Hannon A F, Alexander-Katz A, Ross C A and Berggren K K 2014 Design rules for self-assembled block copolymer patterns using tiled templates Nat Commun 5

[186] Bencher C, Smith J, Miao L, Cai C, Chen Y, Cheng J Y, Sanders D P, Tjio M, Truong H D, Holmes S and Hinsberg W D 2011 Self-assembly patterning for sub-15nm half-pitch: a transition from lab to fab. pp 79700F-F-9

[187] Doerk G S, Cheng J Y, Rettner C T, Balakrishnan S, Arellano N and Sanders D P 2013 Deterministically isolated gratings through the directed self-assembly of block copolymers. pp $86800 \mathrm{Y}-\mathrm{Y}-8$

[188] Tsai H, Pitera J W, Miyazoe H, Bangsaruntip S, Engelmann S U, Liu C-C, Cheng J Y, Bucchignano J J, Klaus D P, Joseph E A, Sanders D P, Colburn M E and Guillorn M A 2014 Two-Dimensional Pattern Formation Using Graphoepitaxy of PS-b-PMMA Block Copolymers for Advanced FinFET Device and Circuit Fabrication ACS Nano 8 5227-32

[189] Doerk G S, Cheng J Y, Singh G, Rettner C T, Pitera J W, Balakrishnan S, Arellano N and Sanders D P 2014 Enabling complex nanoscale pattern customization using directed selfassembly Nat Commun $\mathbf{5}$

[190] Millward D B, Lugani G S, Light S L, Niroomand A, Hustad P D, Trefonas P, Quach D and Ginzburg V V 2015 Graphoepitaxial and chemoepitaxial methods for creating linespace patterns at 33nm pitch: comparison to a HVM process. pp 942304--12

[191] Quach D, Ginzburg V V, Li M, Wu J, Chang S-w, Trefonas P, Hustad P D, Millward D B, Lugani G S and Light S L 2015 Impact of materials selection on graphoepitaxial directed self-assembly for line-space patterning. pp 94230N-N-9

[192] You B K, Park W I, Kim J M, Park K-I, Seo H K, Lee J Y, Jung Y S and Lee K J 2014 Reliable Control of Filament Formation in Resistive Memories by Self-Assembled Nanoinsulators Derived from a Block Copolymer ACS Nano $89492-502$ 
[193] Frascaroli J, Brivio S, Ferrarese Lupi F, Seguini G, Boarino L, Perego M and Spiga S 2015 Resistive Switching in High-Density Nanodevices Fabricated by Block Copolymer Self-Assembly ACS Nano 9 2518-29

[194] Park W I, You B K, Mun B H, Seo H K, Lee J Y, Hosaka S, Yin Y, Ross C A, Lee K J and Jung Y S 2013 Self-Assembled Incorporation of Modulated Block Copolymer Nanostructures in Phase-Change Memory for Switching Power Reduction ACS Nano 7 2651-8

[195] Mun B H, You B K, Yang S R, Yoo H G, Kim J M, Park W I, Yin Y, Byun M, Jung Y S and Lee K J 2015 Flexible One Diode-One Phase Change Memory Array Enabled by Block Copolymer Self-Assembly ACS Nano 9 4120-8

[196] Naito K, Hieda H, Sakurai M, Kamata Y and Asakawa K 2002 2.5-inch disk patterned media prepared by an artificially assisted self-assembling method Magnetics, IEEE Transactions on 38 1949-51

[197] Rhys Alun G, Aled W, Chloe O, Jonathan R, Aravind V and Thomas T 2013 Directed self-assembly of block copolymers for use in bit patterned media fabrication Journal of Physics D: Applied Physics 46503001

[198] Hardy C G and Tang C 2013 Advances in square arrays through self-assembly and directed self-assembly of block copolymers J. Polym. Sci., Part B: Polym. Phys. 51 2-15

[199] Park W I, Kim Y, Jeong J W, Kim K, Yoo J-K, Hur Y H, Kim J M, Thomas E L, Alexander-Katz A and Jung Y S 2013 Host-Guest Self-assembly in Block Copolymer Blends Sci. Rep. 3

[200] Shin D O, Mun J H, Hwang G-T, Yoon J M, Kim J Y, Yun J M, Yang Y-B, Oh Y, Lee J Y, Shin J, Lee K J, Park S, Kim J U and Kim S O 2013 Multicomponent Nanopatterns by Directed Block Copolymer Self-Assembly ACS Nano 7 8899-907

[201] Tavakkoli K. G A, Nicaise S M, Hannon A F, Gotrik K W, Alexander-Katz A, Ross C A and Berggren K K 2014 Sacrificial-Post Templating Method for Block Copolymer SelfAssembly Small 10 493-9

[202] Yi H, Bao X-Y, Tiberio R and Wong H S P 2014 A General Design Strategy for Block Copolymer Directed Self-Assembly Patterning of Integrated Circuits Contact Holes using an Alphabet Approach Nano Lett. 15 805-12

[203] Farrell R A, Hosler E R, Schmid G M, Xu J, Preil M E, Rastogi V, Mohanty N, Kumar K, Cicoria M J, Hetzer D R and DeVilliers A 2014 Manufacturability considerations for DSA. pp 90510Z-Z-12

[204] Wan L, Ruiz R, Gao H, Patel K C, Albrecht T R, Yin J, Kim J, Cao Y and Lin G 2015 The Limits of Lamellae-Forming PS-b-PMMA Block Copolymers for Lithography ACS Nano 9 7506-14

[205] Liddle J A and Gallatin G M 2016 Nanomanufacturing: A Perspective ACS Nano

[206] Luchnikov V, Kondyurin A, Formanek P, Lichte H and Stamm M 2007 Moiré Patterns in Superimposed Nanoporous Thin Films Derived from Block-Copolymer Assemblies Nano Lett. 7 3628-32

[207] Jeong J W, Park W I, Do L-M, Park J-H, Kim T-H, Chae G and Jung Y S 2012 Nanotransfer Printing with sub-10 nm Resolution Realized using Directed Self-Assembly Adv. Mater. 24 3526-31

[208] Tavakkoli K. G. A, Gotrik K W, Hannon A F, Alexander-Katz A, Ross C A and Berggren K K 2012 Templating Three-Dimensional Self-Assembled Structures in Bilayer Block Copolymer Films Science 336 1294-8 
[209] Kim J Y, Kim B H, Hwang J O, Jeong S-J, Shin D O, Mun J H, Choi Y J, Jin H M and Kim S O 2013 Flexible and Transferrable Self-Assembled Nanopatterning on Chemically Modified Graphene Adv. Mater. 25 1331-5

[210] Jeong J W, Yang S R, Hur Y H, Kim S W, Baek K M, Yim S, Jang H-I, Park J H, Lee S Y, Park C-O and Jung Y S 2014 High-resolution nanotransfer printing applicable to diverse surfaces via interface-targeted adhesion switching Nat Commun $\mathbf{5}$

[211] Majewski P W and Yager K G 2015 Latent Alignment in Pathway-Dependent Ordering of Block Copolymer Thin Films Nano Lett. 15 5221-8

[212] Dai H J, Balsara N P, Garetz B A and Newstein M C 1996 Grain Growth and Defect Annihilation in Block Copolymers Phys. Rev. Lett. 77 3677-80

[213] Ruiz R, Bosworth J K and Black C T 2008 Effect of structural anisotropy on the coarsening kinetics of diblock copolymer striped patterns Phys. Rev. B 77 054204-5

[214] Hahm J, Lopes W A, Jaeger H M and Sibener S J 1998 Defect evolution in ultrathin films of polystyrene-block-polymethylmethacrylate diblock copolymers observed by atomic force microscopy The Journal of Chemical Physics 109 10111-4

[215] Harrison C, Cheng Z, Sethuraman S, Huse D A, Chaikin P M, Vega D A, Sebastian J M, Register R A and Adamson D H 2002 Dynamics of pattern coarsening in a twodimensional smectic system Phys. Rev. E 66011706

[216] Harrison C, Adamson D H, Cheng Z, Sebastian J M, Sethuraman S, Huse D A, Register R A and Chaikin P M 2000 Mechanisms of Ordering in Striped Patterns Science 290 $1558-60$

[217] Hahm J and Sibener S J 2001 Time-resolved atomic force microscopy imaging studies of asymmetric PS-b-PMMA ultrathin films: Dislocation and disclination transformations, defect mobility, and evolution of nanoscale morphology The Journal of Chemical Physics 114 4730-40

[218] Tong Q and Sibener S J 2013 Visualization of Individual Defect Mobility and Annihilation within Cylinder-Forming Diblock Copolymer Thin Films on Nanopatterned Substrates Macromol. 46 8538-44

[219] Harrison C, Angelescu D E, Trawick M, Zhengdong C, Huse D A, Chaikin P M, Vega D A, Sebastian J M, Register R A and Adamson D H 2004 Pattern coarsening in a 2D hexagonal system EPL (Europhysics Letters) 67800

[220] Black C T and Guarini K W 2004 Structural evolution of cylindrical-phase diblock copolymer thin films J. Polym. Sci., Part A: Polym. Chem. 42 1970-5

[221] Vega D A, Harrison C K, Angelescu D E, Trawick M L, Huse D A, Chaikin P M and Register R A 2005 Ordering mechanisms in two-dimensional sphere-forming block copolymers Phys. Rev. E 71061803

[222] Ruiz R, Sandstrom R L and Black C T 2007 Induced Orientational Order in Symmetric Diblock Copolymer Thin Films Adv. Mater. 19 587-91

[223] Ji S, Liu C-C, Liao W, Fenske A L, Craig G S W and Nealey P F 2011 Domain Orientation and Grain Coarsening in Cylinder-Forming Poly(styrene-b-methyl methacrylate) Films Macromol. 44 4291-300

[224] Ryu H J, Fortner D B, Lee S, Ferebee R, De Graef M, Misichronis K, Avgeropoulos A and Bockstaller M R 2013 Role of Grain Boundary Defects During Grain Coarsening of Lamellar Block Copolymers Macromol. 46 204-15

[225] Kim B, Laachi N, Delaney K T, Carilli M, Kramer E J and Fredrickson G H 2014 Thermodynamic and kinetic aspects of defectivity in directed self-assembly of cylinder- 
forming diblock copolymers in laterally confining thin channels J. Appl. Polym. Sci. 131 40790

[226] Takahashi H, Laachi N, Delaney K T, Hur S-M, Weinheimer C J, Shykind D and Fredrickson G H 2012 Defectivity in Laterally Confined Lamella-Forming Diblock Copolymers: Thermodynamic and Kinetic Aspects Macromol. 45 6253-65

[227] Peters A J, Lawson R A, Nation B D, Ludovice P J and Henderson C L 2015 Coarsegrained molecular dynamics modeling of the kinetics of lamellar BCP defect annealing. pp 94231Y-Y-15

[228] Welander A M, Kang H, Stuen K O, Solak H H, Müller M, de Pablo J J and Nealey P F 2008 Rapid Directed Assembly of Block Copolymer Films at Elevated Temperatures Macromol. 41 2759-61

[229] Yager K G, Fredin N J, Zhang X, Berry B C, Karim A and Jones R L 2010 Evolution of block-copolymer order through a moving thermal zone Soft Matter 6 92-9

[230] Gaik Teoh L, Shieh J, Hao Lai W and Hsiung Hon M 2004 Effects of mesoporous structure on grain growth of nanostructured tungsten oxide J. Mater. Res. 19 2687-93

[231] Han C D, Baek D M, Kim J K, Ogawa T, Sakamoto N and Hashimoto T 1995 Effect of Volume Fraction on the Order-Disorder Transition in Low Molecular Weight Polystyrene-block-Polyisoprene Copolymers. 1. Order-Disorder Transition Temperature Determined by Rheological Measurements Macromol. 28 5043-62

[232] Bates F S, Rosedale J H and Fredrickson G H 1990 Fluctuation effects in a symmetric diblock copolymer near the order-disorder transition The Journal of Chemical Physics 92 6255-70

[233] Stühn B 1992 The relation between the microphase separation transition and the glass transition in diblock copolymers J. Polym. Sci., Part B: Polym. Phys. 30 1013-9

[234] Russell T P, Hjelm R P and Seeger P A 1990 Temperature dependence of the interaction parameter of polystyrene and poly(methyl methacrylate) Macromol. 23 890-3

[235] Zhao Y, Sivaniah E and Hashimoto T 2008 SAXS Analysis of the Order-Disorder Transition and the Interaction Parameter of Polystyrene-block-poly(methyl methacrylate) Macromol. 41 9948-51

[236] Forrey C, Yager K G and Broadaway S P 2011 Molecular Dynamics Study of the Role of the Free Surface on Block Copolymer Thin Film Morphology and Alignment ACS Nano 5 2895-907

[237] Ceresoli M, Volpe F G, Seguini G, Antonioli D, Gianotti V, Sparnacci K, Laus M and Perego M 2015 Scaling of correlation length in lamellae forming PS-b-PMMA thin films upon high temperature rapid thermal treatments Journal of Materials Chemistry $C \mathbf{3}$ 8618-24

[238] Majewski P W and Yager K G 2016 Reordering transitions during annealing of block copolymer cylinder phases Soft Matter 12 281-94

[239] Anderson D A and Freeman E S 1961 The kinetics of the thermal degradation of polystyrene and polyethylene Journal of Polymer Science 54 253-60

[240] Ferriol M, Gentilhomme A, Cochez M, Oget N and Mieloszynski J L 2003 Thermal degradation of poly(methyl methacrylate) (PMMA): modelling of DTG and TG curves Polym. Degrad. Stab. 79 271-81

[241] Azhari S J and Diab M A 1998 Thermal degradation and stability of poly(4vinylpyridine) homopolymer and copolymers of 4-vinylpyridine with methyl acrylate Polym. Degrad. Stab. 60 253-6 
[242] Calahorra E, Cortazar M and Guzmán G M 1985 Thermal decomposition of poly(ethylene oxide), poly(methyl methacrylate), and their mixtures by thermogravimetric method Journal of Polymer Science: Polymer Letters Edition 23 25760

[243] Pielichowski K and Flejtuch K 2005 Non-oxidative thermal degradation of poly(ethylene oxide): kinetic and thermoanalytical study J. Anal. Appl. Pyrolysis 73 131-8

[244] Camino G, Lomakin S M and Lazzari M 2001 Polydimethylsiloxane thermal degradation Part 1. Kinetic aspects Polymer 42 2395-402

[245] Perego M, Ferrarese Lupi F, Ceresoli M, Giammaria T J, Seguini G, Enrico E, Boarino L, Antonioli D, Gianotti V, Sparnacci K and Laus M 2014 Ordering dynamics in symmetric PS-b-PMMA diblock copolymer thin films during rapid thermal processing Journal of Materials Chemistry C 2 6655-64

[246] Giammaria T J, Ferrarese Lupi F, Seguini G, Perego M, Vita F, Francescangeli O, Wenning B, Ober C K, Sparnacci K, Antonioli D, Gianotti V and Laus M 2016 Micrometer-Scale Ordering of Silicon-Containing Block Copolymer Thin Films via High-Temperature Thermal Treatments ACS Applied Materials \& Interfaces 8 9897-908

[247] Dalvi M C and Lodge T P 1993 Parallel and perpendicular chain diffusion in a lamellar block copolymer Macromol. 26 859-61

[248] Hamersky M W, Tirrell M and Lodge T P 1998 Anisotropy of Diffusion in a Lamellar Styrene-Isoprene Block Copolymer Langmuir 14 6974-9

[249] Croll A B, Matsen M W, Shi A-C and Dalnoki-Veress K 2008 Kinetics of layer hopping in a diblock copolymer lamellar phase The European Physical Journal E: Soft Matter and Biological Physics 27 407-11

[250] Hashimoto T, Sakamoto N and Koga T 1996 Nucleation and growth of anisotropic grain in block copolymers near order-disorder transition Phys. Rev. E 54 5832-5

[251] Chastek T Q and Lodge T P 2004 Grain Shapes and Growth Kinetics of the Cylinder Phase in a Block Copolymer Solution Macromol. 37 4891-9

[252] Chastek T Q and Lodge T P 2005 Twinning and growth kinetics of lamellar grains in a diblock copolymer solution J. Polym. Sci., Part B: Polym. Phys. 43 405-12

[253] Chastek T Q and Lodge T P 2006 Grain shapes and growth kinetics during self-assembly of block copolymers J. Polym. Sci., Part B: Polym. Phys. 44 481-91

[254] Fredrickson G H and Binder K 1989 Kinetics of metastable states in block copolymer melts The Journal of Chemical Physics 91 7265-75

[255] Koneripalli N, Singh N, Levicky R, Bates F S, Gallagher P D and Satija S K 1995 Confined Block Copolymer Thin Films Macromol. 28 2897-904

[256] Russell T P, Menelle A, Anastasiadis S H, Satija S K and Majkrzak C F 1991 Unconventional morphologies of symmetric, diblock copolymers due to film thickness constraints Macromol. 24 6263-9

[257] Maaloum M, Ausserre D, Chatenay D and Gallot Y 1993 Spinodal decomposition-like patterns via metastable state relaxation Phys. Rev. Lett. 70 2577-80

[258] Joly S, Ausserré D, Brotons G and Gallot Y 2002 Hole nucleation in thin diblock copolymer films The European Physical Journal E 8 355-63

[259] Kim S, Bates C M, Thio A, Cushen J D, Ellison C J, Willson C G and Bates F S 2013 Consequences of Surface Neutralization in Diblock Copolymer Thin Films ACS Nano 7 9905-19 
[260] Smith A P, Douglas J F, Meredith J C, Amis E J and Karim A 2001 Combinatorial Study of Surface Pattern Formation in Thin Block Copolymer Films Phys. Rev. Lett. 87015503

[261] Maaloum M, Ausserre D, Chatenay D, Coulon G and Gallot Y 1992 Edge profile of relief 2D domains at the free surface of smectic copolymer thin films Phys. Rev. Lett. 68 15758

[262] Stasiak P, McGraw J D, Dalnoki-Veress K and Matsen M W 2012 Step Edges in Thin Films of Lamellar-Forming Diblock Copolymer Macromol. 45 9531-8

[263] Collin B, Chatenay D, Coulon G, Ausserre D and Gallot Y 1992 Ordering of copolymer thin films as revealed by atomic force microscopy Macromol. 25 1621-2

[264] Vignaud G, Gibaud A, Grübel G, Joly S, Ausserré D, Legrand J F and Gallot Y 1998 Ordering of diblock PS-PBMA thin films: An X-ray reflectivity study Physica $B$ : Condensed Matter 248 250-7

[265] Peters R t and Dalnoki-Veress K 2012 Film thickness dependent ordering dynamics of lamellar forming diblock copolymer thin films The European Physical Journal E 35 1-6

[266] Joly S, Raquois A, Paris F, Hamdoun B, Auvray L, Ausserre D and Gallot Y 1996 Early Stage of Spinodal Decomposition in 2D Phys. Rev. Lett. 77 4394-7

[267] Coulon G, Collin B, Ausserre D, Chatenay D and Russell T P 1990 Islands and holes on the free surface of thin diblock copolymer films. I. Characteristics of formation and growth J. Phys. France 51 2801-11

[268] Bassereau P, Brodbreck D, Russell T P, Brown H R and Shull K R 1993 Topological coarsening of symmetric diblock copolymer films: Model 2D systems Phys. Rev. Lett. 71 1716-9

[269] Stein G E, Lee W B, Fredrickson G H, Kramer E J, Li X and Wang J 2007 Thickness Dependent Ordering in Laterally Confined Monolayers of Spherical-Domain Block Copolymers Macromol. 40 5791-800

[270] Stein G E, Cochran E W, Katsov K, Fredrickson G H, Kramer E J, Li X and Wang J 2007 Symmetry Breaking of In-Plane Order in Confined Copolymer Mesophases Phys. Rev. Lett. 98158302

[271] Morkved T L and Jaeger H M 1997 Thickness-induced morphology changes in lamellar diblock copolymer ultrathin films EPL (Europhysics Letters) 40643

[272] Kim H C and Russell T P 2001 Ordering in thin films of asymmetric diblock copolymers J. Polym. Sci., Part B: Polym. Phys. 39 663-8

[273] Ham S, Shin C, Kim E, Ryu D Y, Jeong U, Russell T P and Hawker C J 2008 Microdomain Orientation of PS-b-PMMA by Controlled Interfacial Interactions Macromol. 41 6431-7

[274] Kim S-W, Kim E, Lee H, Berry B C, Kim H-C and Ryu D Y 2015 Thickness-dependent ordering of perpendicularly oriented lamellae in PS-b-PMMA thin films Polymer 74 63-9

[275] Lyakhova K S, Horvat A, Zvelindovsky A V and Sevink G J A 2006 Dynamics of Terrace Formation in a Nanostructured Thin Block Copolymer Film Langmuir 22 584855

[276] Zhang X, De Paoli Lacerda S H, Yager K G, Berry B C, Douglas J F, Jones R L and Karim A 2009 Target Patterns Induced by Fixed Nanoparticles in Block Copolymer Films ACS Nano 3 2115-20

[277] Sivaniah E, Hayashi Y, Matsubara S, Kiyono S, Hashimoto T, Kukunaga K, Kramer E J and Mates T 2005 Symmetric diblock copolymer thin films on rough substrates. Kinetics and structure formation in pure block copolymer thin films Macromol. 38 1837-49 
[278] Park S M, Berry B C, Dobisz E and Kim H C 2009 Observation of surface corrugationinduced alignment of lamellar microdomains in PS-b-PMMA thin films Soft Matter 5 957-61

[279] Chaturvedi U K, Steiner U, Zak O, Krausch G and Klein J 1989 Interfacial structure in polymer mixtures below the critical point Phys. Rev. Lett. 63 616-9

[280] Schmitz F, Virnau P and Binder K 2014 Logarithmic finite-size effects on interfacial free energies: Phenomenological theory and Monte Carlo studies Phys. Rev. E 90012128

[281] Sunday D F and Kline R J 2015 Reducing Block Copolymer Interfacial Widths through Polymer Additives Macromol. 48 679-86

[282] Wang J-Y, Chen W and Russell T P 2008 Ion-Complexation-Induced Changes in the Interaction Parameter and the Chain Conformation of PS-b-PMMA Copolymers Macromol. 41 4904-7

[283] Dai K H and Kramer E J 1994 Determining the temperature-dependent Flory interaction parameter for strongly immiscible polymers from block copolymer segregation measurements Polymer 35 157-61

[284] Lee D H, Kim H Y, Kim J K, Huh J and Ryu D Y 2006 Swelling and Shrinkage of Lamellar Domain of Conformationally Restricted Block Copolymers by Metal Chloride Macromol. 39 2027-30

[285] O'Driscoll S, Demirel G, Farrell R A, Fitzgerald T G, O'Mahony C, Holmes J D and Morris M A 2011 The morphology and structure of PS-b-P4VP block copolymer films by solvent annealing: effect of the solvent parameter Polym. Adv. Technol. 22 915-23

[286] Zhu L, Cheng S Z D, Calhoun B H, Ge Q, Quirk R P, Thomas E L, Hsiao B S, Yeh F and Lotz B 2001 Phase structures and morphologies determined by self-organization, vitrification, and crystallization: confined crystallization in an ordered lamellar phase of PEO-b-PS diblock copolymer Polymer 42 5829-39

[287] Nose T 1995 Coexistence curves of polystyrene/ poly(dimethylsiloxane) blends Polymer 36 2243-8

[288] Son J G, Gotrik K W and Ross C A 2012 High-Aspect-Ratio Perpendicular Orientation of PS-b-PDMS Thin Films under Solvent Annealing ACS Macro Letters 1 1279-84

[289] Murphy J N, Harris K D and Buriak J M 2015 Automated Defect and Correlation Length Analysis of Block Copolymer Thin Film Nanopatterns PLoS ONE 10 e0133088

[290] Choi K-J, Spruiell J E and White J L 1982 Orientation and morphology of high-density polyethylene film produced by the tubular blowing method and its relationship to process conditions Journal of Polymer Science: Polymer Physics Edition 20 27-47

[291] Qiang Z, Zhang Y, Groff J A, Cavicchi K A and Vogt B D 2014 A generalized method for alignment of block copolymer films: solvent vapor annealing with soft shear Soft Matter 10 6068-76

[292] Qiang Z, Zhang L, Stein G E, Cavicchi K A and Vogt B D 2014 Unidirectional Alignment of Block Copolymer Films Induced by Expansion of a Permeable Elastomer during Solvent Vapor Annealing Macromol. 47 1109-16

[293] Ullal C K, Schmidt R, Hell S W and Egner A 2009 Block Copolymer Nanostructures Mapped by Far-Field Optics Nano Lett. 9 2497-500

[294] Ullal C K, Primpke S, Schmidt R, Böhm U, Egner A, Vana P and Hell S W 2011 Flexible Microdomain Specific Staining of Block Copolymers for 3D Optical Nanoscopy Macromol. 44 7508-10 
[295] Pollard B, Muller E A, Hinrichs K and Raschke M B 2014 Vibrational nanospectroscopic imaging correlating structure with intermolecular coupling and dynamics Nat Commun 5

[296] Hell S W 2007 Far-Field Optical Nanoscopy Science 316 1153-8

[297] Schermelleh L, Heintzmann R and Leonhardt H 2010 A guide to super-resolution fluorescence microscopy The Journal of Cell Biology 190 165-75

[298] Hexemer A, Stein G E, Kramer E J and Magonov S 2005 Block Copolymer Monolayer Structure Measured with Scanning Force Microscopy Moiré Patterns Macromol. 38 7083-9

[299] Hobbs J K and Register R A 2006 Imaging Block Copolymer Crystallization in Real Time with the Atomic Force Microscope Macromol. 39 703-10

[300] Kim B H, Lee H M, Lee J-H, Son S-W, Jeong S-J, Lee S, Lee D I, Kwak S U, Jeong H, Shin H, Yoon J-B, Lavrentovich O D and Kim S O 2009 Spontaneous Lamellar Alignment in Thickness-Modulated Block Copolymer Films Advanced Functional Materials 19 2584-91

[301] Harrison C, Chaikin P M, Huse D A, Register R A, Adamson D H, Daniel A, Huang E, Mansky P, Russell T P, Hawker C J, Egolf D A, Melnikov I V and Bodenschatz E 2000 Reducing Substrate Pinning of Block Copolymer Microdomains with a Buffer Layer of Polymer Brushes Macromol. 33 857-65

[302] Edwards E W, Stoykovich M P, Solak H H and Nealey P F 2006 Long-Range Order and Orientation of Cylinder-Forming Block Copolymers on Chemically Nanopatterned Striped Surfaces Macromol. 39 3598-607

[303] Ruland W and Tompa H 1968 The effect of preferred orientation on the intensity distribution of (hk) interferences Acta Crystallographica Section A 24 93-9

[304] Ruland W and Smarsly B 2004 SAXS of self-assembled oriented lamellar nanocomposite films: an advanced method of evaluation J. Appl. Crystallogr. 37 575-84

[305] Lemaire B J, Panine P, Gabriel J C P and Davidson P 2002 The measurement by SAXS of the nematic order parameter of laponite gels EPL (Europhysics Letters) $\mathbf{5 9} 55$

[306] Chang M Y, Abuzaina F M, Kim W G, Gupton J P, Garetz B A, Newstein M C, Balsara N P, Yang L, Gido S P, Cohen R E, Boontongkong Y and Bellare A 2002 Analysis of Grain Structure in Partially Ordered Block Copolymers by Depolarized Light Scattering and Transmission Electron Microscopy Macromol. 35 4437-47

[307] Segalman R A, Hexemer A and Kramer E J 2003 Edge Effects on the Order and Freezing of a 2D Array of Block Copolymer Spheres Phys. Rev. Lett. 91196101

[308] Liu C-C, Craig G S W, Kang H, Ruiz R, Nealey P F and Ferrier N J 2010 Practical implementation of order parameter calculation for directed assembly of block copolymer thin films J. Polym. Sci., Part B: Polym. Phys. 48 2589-603

[309] Simão C, Tuchapsky D, Khunsin W, Amann A, Morris M A and Torres C M S 2015 Dimensional and defectivity nanometrology of directed self-assembly patterns physica status solidi (c) 12 267-70

[310] Yager K G, Berry B C, Page K, Patton D, Karim A and Amis E J 2009 Disordered nanoparticle interfaces for directed self-assembly Soft Matter 5 622-8

[311] Zhang X, Yager K G, Fredin N J, Ro H W, Jones R L, Karim A and Douglas J F 2010 Thermally Reversible Surface Morphology Transition in Thin Diblock Copolymer Films ACS Nano 4 3653-60 
[312] Higgins J S and Stein R S 1978 Recent developments in polymer applications of smallangle neutron, X-ray and light scattering J. Appl. Crystallogr. 11 346-75

[313] Chu B and Hsiao B S 2001 Small-Angle X-ray Scattering of Polymers Chem. Rev. 101 $1727-62$

[314] Fratzl P 2003 Small-angle scattering in materials science - a short review of applications in alloys, ceramics and composite materials J. Appl. Crystallogr. 36 397-404

[315] Dubček P 2005 Nanostructure as seen by the SAXS Vacuum 80 92-7

[316] Williams C E, May R P and Guinier A 2007 X-ray Characterization of Materials: WileyVCH Verlag GmbH) pp 211-54

[317] Blanchet C E and Svergun D I 2013 Small-Angle X-Ray Scattering on Biological Macromolecules and Nanocomposites in Solution Annu. Rev. Phys. Chem. 64 37-54

[318] Als-Nielsen J and McMorrow D 2011 Elements of Modern X-ray Physics, Second Edition: John Wiley \& Sons, Ltd.)

[319] Bates F S 1984 Block copolymers near the microphase separation transition. 2. Linear dynamic mechanical properties Macromol. 17 2607-13

[320] Benoit H and Hadziioannou G 1988 Scattering theory and properties of block copolymers with various architectures in the homogeneous bulk state Macromol. 21 1449-64

[321] Nojima S, Kato K, Yamamoto S and Ashida T 1992 Crystallization of block copolymers. 1. Small-angle $\mathrm{x}$-ray scattering study of a $\epsilon$-caprolactone-butadiene diblock copolymer Macromol. 25 2237-42

[322] Pedersen J S 2001 Structure factors effects in small-angle scattering from block copolymer micelles and star polymers The Journal of Chemical Physics 114 2839-46

[323] Stefanou N and Modinos A 1993 Scattering of electromagnetic waves by a disordered two-dimensional array of spheres Journal of Physics: Condensed Matter 58859

[324] Förster S, Timmann A, Konrad M, Schellbach C, Meyer A, Funari S S, Mulvaney P and Knott R 2005 Scattering Curves of Ordered Mesoscopic Materials The Journal of Physical Chemistry B 109 1347-60

[325] Ruland W and Smarsly B 2005 SAXS of self-assembled nanocomposite films with oriented two-dimensional cylinder arrays: an advanced method of evaluation J. Appl. Crystallogr. 38 78-86

[326] Ruland W and Smarsly B M 2007 Two-dimensional small-angle X-ray scattering of selfassembled nanocomposite films with oriented arrays of spheres: determination of lattice type, preferred orientation, deformation and imperfection J. Appl. Crystallogr. 40 409-17

[327] Yager K G, Zhang Y, Lu F and Gang O 2014 Periodic lattices of arbitrary nano-objects: modeling and applications for self-assembled systems J. Appl. Crystallogr. 47 118-29

[328] Senesi A J and Lee B 2015 Small-angle scattering of particle assemblies J. Appl. Crystallogr. 48 1172-82

[329] Scherrer P 1918 Bestimmung der Größe und der inneren Struktur von Kolloidteilchen mittels Röntgenstrahlen Nachrichten von der Gesellschaft der Wissenschaften zu Göttingen, Mathematisch-Physikalische Klasse 1918 98-100

[330] Langford J I and Wilson A J C 1978 Scherrer after sixty years: A survey and some new results in the determination of crystallite size J. Appl. Crystallogr. 11 102-13

[331] Scardi P, Leoni M and Delhez R 2004 Line broadening analysis using integral breadth methods: a critical review J. Appl. Crystallogr. 37 381-90

[332] Smilgies D-M 2009 Scherrer grain-size analysis adapted to grazing-incidence scattering with area detectors J. Appl. Crystallogr. 42 1030-4 
[333] Williamson G K and Hall W H 1953 X-ray line broadening from filed aluminium and wolfram Acta Metallurgica $122-31$

[334] Ungar T, Gubicza J, Ribarik G and Borbely A 2001 Crystallite size distribution and dislocation structure determined by diffraction profile analysis: principles and practical application to cubic and hexagonal crystals J. Appl. Crystallogr. 34 298-310

[335] Rao S and Houska C R 1986 X-ray particle-size broadening Acta Crystallographica Section A 42 6-13

[336] Crist B 1973 Small-angle x-ray scattering of semicrystalline polymers. I. Review of existing models Journal of Polymer Science: Polymer Physics Edition 11 635-61

[337] Hindeleh A M and Hosemann R 1988 Paracrystals representing the physical state of matter Journal of Physics C: Solid State Physics 214155

[338] Eads J L and Millane R P 2001 Diffraction by the ideal paracrystal Acta Crystallographica Section A $\mathbf{5 7}$ 507-17

[339] Prosa T J, Moulton J, Heeger A J and Winokur M J 1999 Diffraction Line-Shape Analysis of Poly(3-dodecylthiophene): A Study of Layer Disorder through the Liquid Crystalline Polymer Transition Macromol. 32 4000-9

[340] Noriega R, Rivnay J, Vandewal K, Koch F P V, Stingelin N, Smith P, Toney M F and Salleo A 2013 A general relationship between disorder, aggregation and charge transport in conjugated polymers Nat. Mater. 12 1038-44

[341] Yager K G and Majewski P W 2014 Metrics of graininess: robust quantification of grain count from the non-uniformity of scattering rings J. Appl. Crystallogr. 47 1855-65

[342] Rokhlenko Y, Gopinadhan M, Osuji C O, Zhang K, O’Hern C S, Larson S R, Gopalan P, Majewski P W and Yager K G 2015 Magnetic Alignment of Block Copolymer Microdomains by Intrinsic Chain Anisotropy Phys. Rev. Lett. 115258302

[343] Smilgies D M, Busch P, Papadakis C M and Posselt D 2002 Characterization of polymer thin films with small-angle X-ray scattering under grazing incidence (GISAXS) Synchrotron Radiation News 15 35-42

[344] Müller-Buschbaum P 2003 Grazing incidence small-angle X-ray scattering: an advanced scattering technique for the investigation of nanostructured polymer films Anal. Bioanal. Chem. 376 3-10

[345] Müller-Buschbaum P 2009 Applications of Synchrotron Light to Scattering and Diffraction in Materials and Life Sciences, ed M Gomez, et al.: Springer Berlin Heidelberg) pp 61-89

[346] Renaud G, Lazzari R and Leroy F 2009 Probing surface and interface morphology with Grazing Incidence Small Angle X-Ray Scattering Surf. Sci. Rep. 64 255-380

[347] Cristofolini L 2014 Synchrotron X-ray techniques for the investigation of structures and dynamics in interfacial systems Curr. Opin. Colloid Interface Sci. 19 228-41

[348] Hexemer A and Muller-Buschbaum P 2015 Advanced grazing-incidence techniques for modern soft-matter materials analysis IUCrJ 2 106-25

[349] Müller-Buschbaum P 2016 GISAXS and GISANS as metrology technique for understanding the 3D morphology of block copolymer thin films European Polymer Journal

[350] Jiang Z, Lee D R, Narayanan S, Wang J and Sinha S K 2011 Waveguide-enhanced grazing-incidence small-angle $\mathrm{X}$-ray scattering of buried nanostructures in thin films Phys. Rev. B 84075440 
[351] Narayanan S, Lee D R, Guico R S, Sinha S K and Wang J 2005 Real-Time Evolution of the Distribution of Nanoparticles in an Ultrathin-Polymer-Film-Based Waveguide Phys. Rev. Lett. 94145504

[352] Lee D R, Hagman A, Li X, Narayanan S, Wang J and Shull K R 2006 Perturbation to the resonance modes by gold nanoparticles in a thin-film-based X-ray waveguide Appl. Phys. Lett. 88153101

[353] Wang J, Bedzyk M J, Penner T L and Caffrey M 1991 Structural studies of membranes and surface layers up to 1,000 A thick using X-ray standing waves Nature 354 377-80

[354] Wang J, Caffrey M, Bedzyk M J and Penner T L 2001 Direct Profiling and Reversibility of Ion Distribution at a Charged Membrane/Aqueous Interface: An X-ray Standing Wave Study Langmuir 17 3671-81

[355] Hu X, Jiang Z, Narayanan S, Jiao X, Sandy A R, Sinha S K, Lurio L B and Lal J 2006 Observation of a low-viscosity interface between immiscible polymer layers Phys. Rev. E 74010602

[356] Singh M A and Groves M N 2009 Depth profiling of polymer films with grazingincidence small-angle X-ray scattering Acta Crystallographica Section A 65 190-201

[357] Saito I, Miyazaki T and Yamamoto K 2015 Depth-Resolved Structure Analysis of Cylindrical Microdomain in Block Copolymer Thin Film by Grazing-Incidence SmallAngle X-ray Scattering Utilizing Low-Energy X-rays Macromol. 48 8190-6

[358] Lee B, Park I, Yoon J, Park S, Kim J, Kim K-W, Chang T and Ree M 2005 Structural Analysis of Block Copolymer Thin Films with Grazing Incidence Small-Angle X-ray Scattering Macromol. 38 4311-23

[359] Park S, Wang J-Y, Kim B, Chen W and Russell T P 2007 Solvent-Induced Transition from Micelles in Solution to Cylindrical Microdomains in Diblock Copolymer Thin Films Macromol. 40 9059-63

[360] Papadakis C M, Di Z, Posselt D and Smilgies D-M 2008 Structural Instabilities in Lamellar Diblock Copolymer Thin Films During Solvent Vapor Uptake Langmuir 24 $13815-8$

[361] Kim T H, Hwang J, Hwang W S, Huh J, Kim H C, Kim S H, Hong J M, Thomas E L and Park C 2008 Hierarchical Ordering of Block Copolymer Nanostructures by Solvent Annealing Combined with Controlled Dewetting Adv. Mater. 20 522-7

[362] Park S, Kim B, Xu J, Hofmann T, Ocko B M and Russell T P 2009 Lateral Ordering of Cylindrical Microdomains Under Solvent Vapor Macromol. 42 1278-84

[363] Paik M Y, Bosworth J K, Smilges D-M, Schwartz E L, Andre X and Ober C K 2010 Reversible Morphology Control in Block Copolymer Films via Solvent Vapor Processing: An in Situ GISAXS Study Macromol. 43 4253-60

[364] Marques D S, Dorin R M, Wiesner U, Smilgies D-M, Behzad A R, Vainio U, Peinemann K-V and Nunes S P 2014 Time-resolved GISAXS and cryo-microscopy characterization of block copolymer membrane formation Polymer 55 1327-32

[365] Gu X, Gunkel I, Hexemer A, Gu W and Russell T P 2014 An In Situ Grazing Incidence X-Ray Scattering Study of Block Copolymer Thin Films During Solvent Vapor Annealing Adv. Mater. 26 273-81

[366] Smilgies D-M and Blasini D R 2007 Indexation scheme for oriented molecular thin films studied with grazing-incidence reciprocal-space mapping J. Appl. Crystallogr. 40 716-8

[367] Breiby D W, Bunk O, Andreasen J W, Lemke H T and Nielsen M M 2008 Simulating Xray diffraction of textured films J. Appl. Crystallogr. 41 262-71 
[368] Baker J L, Widmer-Cooper A, Toney M F, Geissler P L and Alivisatos A P 2010 DeviceScale Perpendicular Alignment of Colloidal Nanorods Nano Lett. 10 195-201

[369] Baker J L, Jimison L H, Mannsfeld S, Volkman S, Yin S, Subramanian V, Salleo A, Alivisatos A P and Toney M F 2010 Quantification of Thin Film Crystallographic Orientation Using X-ray Diffraction with an Area Detector Langmuir 26 9146-51

[370] Johnston D E, Yager K G, Hlaing H, Lu X, Ocko B M and Black C T 2014 Nanostructured Surfaces Frustrate Polymer Semiconductor Molecular Orientation ACS Nano 8 243-9

[371] Lazzari R, Leroy F and Renaud G 2007 Grazing-incidence small-angle x-ray scattering from dense packing of islands on surfaces: Development of distorted wave Born approximation and correlation between particle sizes and spacing Phys. Rev. B 76125411

[372] Toney M F and Brennan S 1989 Observation of the effect of refraction on x rays diffracted in a grazing-incidence asymmetric Bragg geometry Phys. Rev. B 39 7963-6

[373] Smilgies D-M 2002 Geometry-independent intensity correction factors for grazingincidence diffraction Rev Sci Instrum 73 1706-10

[374] Busch P, Rauscher M, Smilgies D-M, Posselt D and Papadakis C M 2006 Grazingincidence small-angle X-ray scattering from thin polymer films with lamellar structures the scattering cross section in the distorted-wave Born approximation J. Appl. Crystallogr. 39 433-42

[375] Yoneda Y 1963 Anomalous Surface Reflection of X Rays Phys Rev 131 2010-3

[376] Vineyard G H 1982 Grazing-incidence diffraction and the distorted-wave approximation for the study of surfaces Phys. Rev. B 26 4146-59

[377] Sinha S K, Sirota E B, Garoff S and Stanley H B 1988 X-Ray and Neutron-Scattering from Rough Surfaces Phys. Rev. B 38 2297-311

[378] Rauscher M, Salditt T and Spohn H 1995 Small-angle x-ray scattering under grazing incidence: The cross section in the distorted-wave Born approximation Phys. Rev. B 52 16855-63

[379] Rauscher M, Paniago R, Metzger H, Kovats Z, Domke J, Peisl J, Pfannes H-D, Schulze J and Eisele I 1999 Grazing incidence small angle x-ray scattering from free-standing nanostructures J. Appl. Phys. 86 6763-9

[380] Tate M P and Hillhouse H W 2007 General method for simulation of 2D GISAXS intensities for any nanostructured film using discrete Fourier transforms Journal of Physical Chemistry C 111 7645-54

[381] de Boer D K G 1996 X-ray scattering and x-ray fluorescence from materials with rough interfaces Phys. Rev. B 53 6048-64

[382] Smilgies D-M, Heitsch A T and Korgel B A 2012 Stacking of Hexagonal Nanocrystal Layers during Langmuir-Blodgett Deposition The Journal of Physical Chemistry B 116 6017-26

[383] Lazzari R 2002 IsGISAXS: a program for grazing-incidence small-angle X-ray scattering analysis of supported islands $J$. Appl. Crystallogr. 35 406-21

[384] Tate M P, Urade V N, Kowalski J D, Wei T C, Hamilton B D, Eggiman B W and Hillhouse H W 2006 Simulation and interpretation of 2D diffraction patterns from selfassembled nanostructured films at arbitrary angles of incidence: From grazing incidence (above the critical angle) to transmission perpendicular to the substrate $J$ Phys Chem $B$ $1109882-92$ 
[385] Sarje A, Li X S, Chourou S, Chan E R and Hexemer A 2012 Massively parallel X-ray scattering simulations. In: Proceedings of the International Conference on High Performance Computing, Networking, Storage and Analysis, (Salt Lake City, Utah: IEEE Computer Society Press) pp 1-11

[386] Chourou S T, Sarje A, Li X S, Chan E R and Hexemer A 2013 HipGISAXS: a highperformance computing code for simulating grazing-incidence X-ray scattering data $J$. Appl. Crystallogr. 46 1781-95

[387] Hailey A K, Hiszpanski A M, Smilgies D-M and Loo Y-L 2014 The Diffraction Pattern Calculator (DPC) toolkit: a user-friendly approach to unit-cell lattice parameter identification of two-dimensional grazing-incidence wide-angle X-ray scattering data $J$. Appl. Crystallogr. 47 2090-9

[388] Jiang Z 2015 GIXSGUI: a MATLAB toolbox for grazing-incidence X-ray scattering data visualization and reduction, and indexing of buried three-dimensional periodic nanostructured films J. Appl. Crystallogr. 48 917-26

[389] Durniak C, Ganeva M, Pospelov G, Van Herck W and Wuttke J 2015 BornAgain Software for simulating and fitting X-ray and neutron small-angle scattering at grazing incidence.

[390] Jones R L, Hu T, Lin E K, Wu W-L, Kolb R, Casa D M, Bolton P J and Barclay G G 2003 Small angle x-ray scattering for sub-100 nm pattern characterization Appl. Phys. Lett. 83 4059-61

[391] Jones R L, Hu T, Soles C L, Lin E K, Wu W-1, Casa D M and Mahorowala A 2004 Preliminary evaluation of line-edge roughness metrology based on CD-SAXS Proc. SPIE 5375 191-8

[392] Hu T, Jones R L, Wu W-1, Lin E K, Lin Q, Keane D, Weigand S and Quintana J 2004 Small angle $\mathrm{x}$-ray scattering metrology for sidewall angle and cross section of nanometer scale line gratings $J$. Appl. Phys. 96 1983-7

[393] Wang C, Jones R L, Lin E K, Wu W-L, Rice B J, Choi K-W, Thompson G, Weigand S J and Keane D T 2007 Characterization of correlated line edge roughness of nanoscale line gratings using small angle x-ray scattering J. Appl. Phys. 102024901

[394] Wang C, Jones R L, Lin E K, Wu W-L and Leu J 2007 Small angle x-ray scattering measurements of lithographic patterns with sidewall roughness from vertical standing waves Appl. Phys. Lett. 90193122

[395] Wang C, Choi K-W, Fu W-E, Ho D L, Jones R L, Soles C, Lin E K, Wu W-1, Clarke J S and Bunday B 2008 CD-SAXS measurements using laboratory-based and synchrotronbased instruments Proc. SPIE 6922 69222E

[396] Wang C, Jones R L, Lin E K, Wu W-l, Villarrubia J S, Choi K-W, Clarke J S, Rice B J, Leeson M J, Roberts J, Bristol R and Bunday B 2007 Line edge roughness characterization of sub-50nm structures using CD-SAXS: round-robin benchmark results Proc. SPIE 6518651810

[397] Wang C, Choi K-W, Chen Y-C, Price J, Ho D L, Jones R L, Soles C, Lin E K, Wu W-1 and Bunday B D 2009 Nonplanar high-k dielectric thickness measurements using CDSAXS Proc. SPIE 7272 72722M

[398] Settens C, Cordes A, Bunday B, Bello A, Kamineni V, Paul A, Fronheiser J and Matyi R 2014 Assessment of critical dimension small-angle x-ray scattering measurement approaches for FinFET fabrication process monitoring MOEMS 13 041408- 
[399] Sunday D F, List S, Chawla J S and Kline R J 2015 Determining the shape and periodicity of nanostructures using small-angle X-ray scattering J. Appl. Crystallogr. 48

[400] Jones R L, Soles C L, Lin E K, Hu W, Reano R M, Pang S W, Weigand S J, Keane D T and Quintana J P 2006 Pattern fidelity in nanoimprinted films using critical dimension small angle x-ray scattering MOEMS 5 013001--7

[401] Jones R L, Hu T, Soles C L, Lin E K, Reano R M, Pang S W and Casa D M 2006 RealTime Shape Evolution of Nanoimprinted Polymer Structures during Thermal Annealing Nano Lett. 6 1723-8

[402] Sunday D F, Hammond M R, Wang C, Wu W-1, Kline R J and Stein G E 2013 Threedimensional $\mathrm{x}$-ray metrology for block copolymer lithography line-space patterns MOEMS 12031103

[403] Sunday D F, Hammond M R, Wang C, Wu W-1, Delongchamp D M, Tjio M, Cheng J Y, Pitera J W and Kline R J 2014 Determination of the Internal Morphology of Nanostructures Patterned by Directed Self Assembly ACS Nano 8 8426-37

[404] Kulkarni M M, Yager K G, Sharma A and Karim A 2012 Combinatorial Block Copolymer Ordering on Tunable Rough Substrates Macromol. 45 4303-14

[405] Zhang X, Douglas J F, Satija S and Karim A 2015 Enhanced vertical ordering of block copolymer films by tuning molecular mass RSC Advances 5 32307-18

[406] Stein G E, Kramer E J, Li X and Wang J 2007 Single-Crystal Diffraction from TwoDimensional Block Copolymer Arrays Phys. Rev. Lett. 98086101

[407] Singh G, Yager K G, Berry B, Kim H-C and Karim A 2012 Dynamic Thermal FieldInduced Gradient Soft-Shear for Highly Oriented Block Copolymer Thin Films ACS Nano 6 10335-42

[408] Lu X, Yager K G, Johnston D, Black C T and Ocko B M 2013 Grazing-incidence transmission X-ray scattering: surface scattering in the Born approximation J. Appl. Crystallogr. 46 165-72

[409] Mahadevapuram N, Strzalka J and Stein G E 2013 Grazing-incidence transmission small angle X-ray scattering from thin films of block copolymers J. Polym. Sci., Part B: Polym. Phys. 51 602-10

[410] Weidman M C, Yager K G and Tisdale W A 2015 Interparticle Spacing and Structural Ordering in Superlattice PbS Nanocrystal Solids Undergoing Ligand Exchange Chem. Mater. 27 474-82

[411] Mitchell G E, Landes B G, Lyons J, Kern B J, Devon M J, Koprinarov I, Gullikson E M and Kortright J B 2006 Molecular bond selective x-ray scattering for nanoscale analysis of soft matter Appl. Phys. Lett. 89044101

[412] Araki T, Ade H, Stubbs J M, Sundberg D C, Mitchell G E, Kortright J B and Kilcoyne A L D 2006 Resonant soft x-ray scattering from structured polymer nanoparticles Appl. Phys. Lett. 89124106

[413] Stein G E, Liddle J A, Aquila A L and Gullikson E M 2010 Measuring the Structure of Epitaxially Assembled Block Copolymer Domains with Soft X-ray Diffraction Macromol. 43 433-41

[414] Wang C, Hexemer A, Nasiatka J, Chan E R, Young A T, Padmore H A, Schlotter W F, Lüning J, Swaraj S, Watts B, Gann E, Yan H and Ade H 2010 Resonant Soft X-ray Scattering of Polymers with a 2D Detector: Initial Results and System Developments at the Advanced Light Source IOP Conference Series: Materials Science and Engineering 14012016 
[415] Collins B A, Cochran J E, Yan H, Gann E, Hub C, Fink R, Wang C, Schuettfort T, McNeill C R, Chabinyc M L and Ade H 2012 Polarized X-ray scattering reveals noncrystalline orientational ordering in organic films Nat. Mater. 11 536-43

[416] Cheng J Y, Ross C A, Thomas E L, Smith H I and Vancso G J 2002 Fabrication of nanostructures with long-range order using block copolymer lithography Appl. Phys. Lett. $813657-9$

[417] La Y-H, Edwards E W, Park S-M and Nealey P F 2005 Directed Assembly of CylinderForming Block Copolymer Films and Thermochemically Induced Cylinder to Sphere Transition: A Hierarchical Route to Linear Arrays of Nanodots Nano Lett. 5 1379-84

[418] Kang H, Detcheverry F A, Mangham A N, Stoykovich M P, Daoulas K C, Hamers R J, Müller M, de Pablo J J and Nealey P F 2008 Hierarchical Assembly of Nanoparticle Superstructures from Block Copolymer-Nanoparticle Composites Phys. Rev. Lett. 100 148303

[419] Jeong S-J, Kim J E, Moon H-S, Kim B H, Kim S M, Kim J B and Kim S O 2009 Soft Graphoepitaxy of Block Copolymer Assembly with Disposable Photoresist Confinement Nano Lett. $92300-5$

[420] Jeong S-J, Moon H-S, Shin J, Kim B H, Shin D O, Kim J Y, Lee Y-H, Kim J U and Kim S O 2010 One-Dimensional Metal Nanowire Assembly via Block Copolymer Soft Graphoepitaxy Nano Lett. 10 3500-5

[421] Tran H, Ronaldson K, Bailey N A, Lynd N A, Killops K L, Vunjak-Novakovic G and Campos L M 2014 Hierarchically Ordered Nanopatterns for Spatial Control of Biomolecules ACS Nano 8 11846-53

[422] Jung H, Woo S, Park S, Lee S, Kang M, Choe Y, Son J G, Ryu D Y, Huh J and Bang J 2015 Combined epitaxial self-assembly of block copolymer lamellae on a hexagonal prepattern within microgrooves Soft Matter 11 4242-50

[423] Park S-M, Liang X, Harteneck B D, Pick T E, Hiroshiba N, Wu Y, Helms B A and Olynick D L 2011 Sub-10 nm Nanofabrication via Nanoimprint Directed Self-Assembly of Block Copolymers ACS Nano 5 8523-31

[424] Voet V S D, Pick T E, Park S-M, Moritz M, Hammack A T, Urban J J, Ogletree D F, Olynick D L and Helms B A 2011 Interface Segregating Fluoralkyl-Modified Polymers for High-Fidelity Block Copolymer Nanoimprint Lithography J. Am. Chem. Soc. 133 2812-5

[425] Claudia S, Worawut K, Nikolaos K, Mathieu S, Marc Z, Michael A M and Clivia M S T 2014 Order quantification of hexagonal periodic arrays fabricated by in situ solventassisted nanoimprint lithography of block copolymers Nanotechnology 25175703

[426] Li H-W and Huck W T S 2004 Ordered Block-Copolymer Assembly Using Nanoimprint Lithography Nano Lett. 4 1633-6

[427] Luo M, Scott D M and Epps T H 2015 Writing Highly Ordered Macroscopic Patterns in Cylindrical Block Polymer Thin Films via Raster Solvent Vapor Annealing and Soft Shear ACS Macro Letters 4 516-20

[428] Singer J P, Gotrik K W, Lee J-H, Kooi S E, Ross C A and Thomas E L 2014 Alignment and reordering of a block copolymer by solvent-enhanced thermal laser direct write Polymer 55 1875-82

[429] Croll A B, Massa M V, Matsen M W and Dalnoki-Veress K 2006 Droplet Shape of an Anisotropic Liquid Phys. Rev. Lett. 97204502 
[430] McGraw J D, Rowe I D W, Matsen M W and Dalnoki-Veress K 2011 Dynamics of interacting edge defects in copolymer lamellae The European Physical Journal E 34 1-7

[431] Onses M S, Song C, Williamson L, Sutanto E, Ferreira P M, Alleyne A G, Nealey P F, Ahn H and Rogers J A 2013 Hierarchical patterns of three-dimensional block-copolymer films formed by electrohydrodynamic jet printing and self-assembly Nat Nano 8 667-75

[432] Onses M S, Ramírez-Hernández A, Hur S-M, Sutanto E, Williamson L, Alleyne A G, Nealey P F, de Pablo J J and Rogers J A 2014 Block Copolymer Assembly on Nanoscale Patterns of Polymer Brushes Formed by Electrohydrodynamic Jet Printing ACS Nano 8 6606-13

[433] Nozue Y, Shinohara Y and Amemiya Y 2007 Application of Microbeam Small- and Wide-angle X-ray Scattering to Polymeric Material Characterization Polym. J 391221 37

[434] Ice G E, Budai J D and Pang J W L 2011 The Race to X-ray Microbeam and Nanobeam Science Science 334 1234-9

[435] Burkhard K, Pierre T, Alessandra G and Maya K 2011 Transmission and emission x-ray microscopy: operation modes, contrast mechanisms and applications Journal of Physics: Condensed Matter 23083002

[436] Nugent K A 2009 Coherent methods in the X-ray sciences Advances in Physics 59 1-99

[437] Chapman H N and Nugent K A 2010 Coherent lensless X-ray imaging Nat Photon 4 8339

[438] Robinson I 2012 Nanoparticle Structure by Coherent X-ray Diffraction J. Phys. Soc. Jpn. 82021012

[439] van Zoelen W, Asumaa T, Ruokolainen J, Ikkala O and ten Brinke G 2008 Phase Behavior of Solvent Vapor Annealed Thin Films of PS-b-P4VP(PDP) Supramolecules Macromol. 41 3199-208

[440] Nandan B, Vyas M K, Böhme M and Stamm M 2010 Composition-Dependent Morphological Transitions and Pathways in Switching of Fine Structure in Thin Films of Block Copolymer Supramolecular Assemblies Macromol. 43 2463-73

[441] Di Z, Posselt D, Smilgies D-M and Papadakis C M 2010 Structural Rearrangements in a Lamellar Diblock Copolymer Thin Film during Treatment with Saturated Solvent Vapor Macromol. 43 418-27

[442] Sinturel C, Vayer M, Morris M and Hillmyer M A 2013 Solvent Vapor Annealing of Block Polymer Thin Films Macromol. 46 5399-415

[443] Knoll A, Magerle R and Krausch G 2004 Phase behavior in thin films of cylinderforming ABA block copolymers: Experiments The Journal of Chemical Physics 120 1105-16

[444] Peng J, Xuan Y, Wang H, Li B and Han Y 2005 Solvent vapor induced dewetting in diblock copolymer thin films Polymer 46 5767-72

[445] Peng J, Kim D H, Knoll W, Xuan Y, Li B and Han Y 2006 Morphologies in solventannealed thin films of symmetric diblock copolymer The Journal of Chemical Physics 125064702

[446] Kim G and Libera M 1998 Kinetic constraints on the development of surface microstructure in SBS thin films Macromol. 31 2670-2

[447] Ho R-M, Tseng W-H, Fan H-W, Chiang Y-W, Lin C-C, Ko B-T and Huang B-H 2005 Solvent-induced microdomain orientation in polystyrene-b-poly(l-lactide) diblock copolymer thin films for nanopatterning Polymer 46 9362-77 
[448] Phillip W A, Hillmyer M A and Cussler E L 2010 Cylinder Orientation Mechanism in Block Copolymer Thin Films Upon Solvent Evaporation Macromol. 43 7763-70

[449] Baruth A, Rodwogin M D, Shankar A, Erickson M J, Hillmyer M A and Leighton C 2011 Non-lift-off Block Copolymer Lithography of $25 \mathrm{~nm}$ Magnetic Nanodot Arrays ACS Applied Materials \& Interfaces 3 3472-81

[450] Albert J N L, Young W-S, Lewis R L, Bogart T D, Smith J R and Epps T H 2012 Systematic Study on the Effect of Solvent Removal Rate on the Morphology of Solvent Vapor Annealed ABA Triblock Copolymer Thin Films ACS Nano 6 459-66

[451] Wu Y-H, Lo T-Y, She M-S and Ho R-M 2015 Morphological Evolution of GyroidForming Block Copolymer Thin Films with Varying Solvent Evaporation Rate ACS Applied Materials \& Interfaces 7 16536-47

[452] Kim K, Park S, Kim Y, Bang J, Park C and Ryu D Y 2016 Optimized Solvent Vapor Annealing for Long-Range Perpendicular Lamellae in PS-b-PMMA Films Macromol. 49 1722-30

[453] Kim S H, Misner M J, Xu T, Kimura M and Russell T P 2004 Highly oriented and ordered arrays from block copolymers via solvent evaporation Adv. Mater. 16 226-+

[454] Jung Y S and Ross C A 2009 Solvent-Vapor-Induced Tunability of Self-Assembled Block Copolymer Patterns Adv. Mater. 21 2540-5

[455] Zettl U, Knoll A and Tsarkova L 2010 Effect of Confinement on the Mesoscale and Macroscopic Swelling of Thin Block Copolymer Films Langmuir 26 6610-7

[456] Gotrik K W, Hannon A F, Son J G, Keller B, Alexander-Katz A and Ross C A 2012 Morphology Control in Block Copolymer Films Using Mixed Solvent Vapors ACS Nano 6 8052-9

[457] Kim J M, Kim Y, Park W I, Hur Y H, Jeong J W, Sim D M, Baek K M, Lee J H, Kim MJ and Jung Y S 2015 Eliminating the Trade-Off between the Throughput and Pattern Quality of Sub-15 nm Directed Self-Assembly via Warm Solvent Annealing Advanced Functional Materials 25 306-15

[458] Hayat J, Mitra I, Qiao Y, Stein G E and Tang C 2015 Improving humidity-controlled solvent annealing processes for block copolymer poly(ethylene oxide)-b-polystyrene European Polymer Journal 71 476-89

[459] Kim E, Ahn H, Park S, Lee H, Lee M, Lee S, Kim T, Kwak E-A, Lee J H, Lei X, Huh J, Bang J, Lee B and Ryu D Y 2013 Directed Assembly of High Molecular Weight Block Copolymers: Highly Ordered Line Patterns of Perpendicularly Oriented Lamellae with Large Periods ACS Nano 7 1952-60

[460] Arges C G, Kambe Y, Suh H S, Ocola L E and Nealey P F 2016 Perpendicularly Aligned, Anion Conducting Nanochannels in Block Copolymer Electrolyte Films Chem. Mater. 28 1377-89

[461] Huang W-H, Chen P-Y and Tung S-H 2012 Effects of Annealing Solvents on the Morphology of Block Copolymer-Based Supramolecular Thin Films Macromol. 45 15629

[462] Gowd E B, Koga T, Endoh M K, Kumar K and Stamm M 2014 Pathways of cylindrical orientations in PS-b-P4VP diblock copolymer thin films upon solvent vapor annealing Soft Matter 10 7753-61

[463] Cummins C, Gangnaik A, Kelly R A, Borah D, O'Connell J, Petkov N, Georgiev Y M, Holmes J D and Morris M A 2015 Aligned silicon nanofins via the directed self-assembly 
of PS-b-P4VP block copolymer and metal oxide enhanced pattern transfer Nanoscale 7 6712-21

[464] Bai W, Hannon A F, Gotrik K W, Choi H K, Aissou K, Liontos G, Ntetsikas K, Alexander-Katz A, Avgeropoulos A and Ross C A 2014 Thin Film Morphologies of Bulk-Gyroid Polystyrene-block-polydimethylsiloxane under Solvent Vapor Annealing Macromol. 47 6000-8

[465] Park W I, Choi Y J, Yun J M, Hong S W, Jung Y S and Kim K H 2015 Enhancing the Directed Self-assembly Kinetics of Block Copolymers Using Binary Solvent Mixtures ACS Applied Materials \& Interfaces 7 25843-50

[466] Peng J, Han Y, Knoll W and Kim D H 2007 Development of Nanodomain and Fractal Morphologies in Solvent Annealed Block Copolymer Thin Films Macromol. Rapid Commun. 28 1422-8

[467] Vayer M, Hillmyer M A, Dirany M, Thevenin G, Erre R and Sinturel C 2010 Perpendicular orientation of cylindrical domains upon solvent annealing thin films of polystyrene-b-polylactide Thin Solid Films 518 3710-5

[468] Zhang X, Murphy J N, Wu N L Y, Harris K D and Buriak J M 2011 Rapid Assembly of Nanolines with Precisely Controlled Spacing from Binary Blends of Block Copolymers Macromol. 44 9752-7

[469] Park S, Lee D H, Xu J, Kim B, Hong S W, Jeong U, Xu T and Russell T P 2009 Macroscopic 10-Terabit-per-Square-Inch Arrays from Block Copolymers with Lateral Order Science 323 1030-3

[470] Seguini G, Zanenga F, Giammaria T J, Ceresoli M, Sparnacci K, Antonioli D, Gianotti V, Laus M and Perego M 2016 Enhanced Lateral Ordering in Cylinder Forming PS-bPMMA Block Copolymers Exploiting the Entrapped Solvent ACS Applied Materials \& Interfaces 8 8280-8

[471] Zhang X, Yager K G, Kang S, Fredin N J, Akgun B, Satija S, Douglas J F, Karim A and Jones R L 2009 Solvent Retention in Thin Spin-Coated Polystyrene and Poly(methyl methacrylate) Homopolymer Films Studied By Neutron Reflectometry Macromol. 43 $1117-23$

[472] Lee J H, Kim Y, Cho J-Y, Yang S R, Kim J M, Yim S, Lee H and Jung Y S 2015 In Situ Nanolithography with Sub-10 nm Resolution Realized by Thermally Assisted SpinCasting of a Self-Assembling Polymer Adv. Mater. 27 4814-22

[473] Park W I, Kim J M, Jeong J W and Jung Y S 2014 Deep-Nanoscale Pattern Engineering by Immersion-Induced Self-Assembly ACS Nano 8 10009-18

[474] Modi A, Bhaway S M, Vogt B D, Douglas J F, Al-Enizi A, Elzatahry A, Sharma A and Karim A 2015 Direct Immersion Annealing of Thin Block Copolymer Films ACS Applied Materials \& Interfaces 7 21639-45

[475] Chang S-w, Evans J P, Ge S, Ginzburg V V, Kramer J W, Landes B, Lee C, Meyers G F, Murray D J, Park J, Sharma R, Trefonas P, Weinhold J D, Zhang J and Hustad P D 2013 New materials and processes for directed self-assembly. pp 86800F-F-9

[476] Song D-P, Li C, Colella N S, Xie W, Li S, Lu X, Gido S, Lee J-H and Watkins J J 2015 Large-Volume Self-Organization of Polymer/Nanoparticle Hybrids with Millimeter-Scale Grain Sizes Using Brush Block Copolymers J. Am. Chem. Soc. 137 12510-3

[477] Whitmore M D and Vavasour J D 1992 Self-consistent mean field theory of the microphase diagram of block copolymer/neutral solvent blends Macromol. 25 2041-5 
[478] Dudowicz J, Freed K F and Douglas J F 1995 Modification of the Phase Stability of Polymer Blends by Diblock Copolymer Additives Macromol. 28 2276-87

[479] Huang C-I and Lodge T P 1998 Self-Consistent Calculations of Block Copolymer Solution Phase Behavior Macromol. 31 3556-65

[480] Bosse A W, Tirumala V R and Lin E K 2009 Tuning block copolymer phase behavior with a selectively associating homopolymer additive J. Polym. Sci., Part B: Polym. Phys. 47 2083-90

[481] Wang J-Y, Chen W, Roy C, Sievert J D and Russell T P 2008 Influence of Ionic Complexes on Phase Behavior of Polystyrene-b-poly(methyl methacrylate) Copolymers Macromol. 41 963-9

[482] Tirumala V R, Daga V, Bosse A W, Romang A, Ilavsky J, Lin E K and Watkins J J 2008 Well-Ordered Polymer Melts with $5 \mathrm{~nm}$ Lamellar Domains from Blends of a Disordered Block Copolymer and a Selectively Associating Homopolymer of Low or High Molar Mass Macromol. 41 7978-85

[483] Tirumala V R, Romang A, Agarwal S, Lin E K and Watkins J J 2008 Well Ordered Polymer Melts from Blends of Disordered Triblock Copolymer Surfactants and Functional Homopolymers Adv. Mater. 20 1603-8

[484] Kim B H, Park S J, Jin H M, Kim J Y, Son S-W, Kim M-H, Koo C M, Shin J, Kim J U and Kim S O 2015 Anomalous Rapid Defect Annihilation in Self-Assembled Nanopatterns by Defect Melting Nano Lett. 15 1190-6

[485] Listak J and Bockstaller M R 2006 Stabilization of Grain Boundary Morphologies in Lamellar Block Copolymer/Nanoparticle Blends Macromol. 39 5820-5

[486] Ploshnik E, Langner K M, Halevi A, Ben-Lulu M, Müller A H E, Fraaije J G E M, Agur Sevink G J and Shenhar R 2013 Hierarchical Structuring in Block Copolymer Nanocomposites through Two Phase-Separation Processes Operating on Different Time Scales Advanced Functional Materials 23 4215-26

[487] Shelton C K and Epps T H 2016 Mapping Substrate Surface Field Propagation in Block Polymer Thin Films Macromol. 49 574-80

[488] Mansky P, Russell T P, Hawker C J, Mays J, Cook D C and Satija S K 1997 Interfacial Segregation in Disordered Block Copolymers: Effect of Tunable Surface Potentials Phys. Rev. Lett. 79237

[489] Huang E, Rockford L, Russell T P and Hawker C J 1998 Nanodomain control in copolymer thin films Nature 395757

[490] Mansky P, Liu Y, Huang E, Russell T P and Hawker C 1997 Controlling PolymerSurface Interactions with Random Copolymer Brushes Science 275 1458-60

[491] Mansky P, Russell T P, Hawker C J, Pitsikalis M and Mays J 1997 Ordered Diblock Copolymer Films on Random Copolymer Brushes Macromol. 30 6810-3

[492] Han E, Stuen K O, Leolukman M, Liu C-C, Nealey P F and Gopalan P 2009 Perpendicular Orientation of Domains in Cylinder-Forming Block Copolymer Thick Films by Controlled Interfacial Interactions Macromol. 42 4896-901

[493] Peters R D, Yang X M, Kim T K and Nealey P F 2000 Wetting Behavior of Block Copolymers on Self-Assembled Films of Alkylchlorosiloxanes: Effect of Grafting Density Langmuir 16 9620-6

[494] Jönsson U, Ivarsson B, Lundström I and Berghem L 1982 Adsorption behavior of fibronectin on well-characterized silica surfaces Journal of Colloid and Interface Science 90 148-63 
[495] Elwing H, Welin S, Askendal A, Nilsson U and LundstrÖm I 1987 A wettability gradient method for studies of macromolecular interactions at the liquid/solid interface Journal of Colloid and Interface Science 119 203-10

[496] Chaudhury M K and Whitesides G M 1992 How to Make Water Run Uphill Science 256 1539-41

[497] Liedberg B and Tengvall P 1995 Molecular Gradients of $\omega$-Substituted Alkanethiols on Gold: Preparation and Characterization Langmuir 11 3821-7

[498] Genzer J and Kramer E J 1998 Pretransitional thinning of a polymer wetting layer EPL (Europhysics Letters) 44180

[499] Smith A P, Sehgal A, Douglas J F, Karim A and Amis E J 2003 Combinatorial Mapping of Surface Energy Effects on Diblock Copolymer Thin Film Ordering Macromol. Rapid Commun. 24 131-5

[500] Kim T K, Yang X M, Peters R D, Sohn B H and Nealey P F 2000 Chemical Modification of Self-Assembled Monolayers by Exposure to Soft X-rays in Air The Journal of Physical Chemistry B 104 7403-10

[501] Peters R D, Yang X M, Kim T K, Sohn B H and Nealey P F 2000 Using Self-Assembled Monolayers Exposed to X-rays To Control the Wetting Behavior of Thin Films of Diblock Copolymers Langmuir 16 4625-31

[502] Berry B C, Stafford C M, Pandya M, Lucas L A, Karim A and Fasolka M J 2007 Versatile platform for creating gradient combinatorial libraries via modulated light exposure Rev Sci Instrum 78072202

[503] Mahadevapuram N, Mitra I, Sridhar S, Strzalka J and Stein G E 2016 Ordering of lamellar block copolymers on oxidized silane coatings European Polymer Journal 75 495-503

[504] Han E, Stuen K O, La Y-H, Nealey P F and Gopalan P 2008 Effect of Composition of Substrate-Modifying Random Copolymers on the Orientation of Symmetric and Asymmetric Diblock Copolymer Domains Macromol. 41 9090-7

[505] Mansky P, haikin P and Thomas E L 1995 Monolayer films of diblock copolymer microdomains for nanolithographic applications J. Mater. Sci. 30 1987-92

[506] Yang X M, Peters R D, Nealey P F, Solak H H and Cerrina F 2000 Guided SelfAssembly of Symmetric Diblock Copolymer Films on Chemically Nanopatterned Substrates Macromol. 33 9575-82

[507] Kim S O, Solak H H, Stoykovich M P, Ferrier N J, de Pablo J J and Nealey P F 2003 Epitaxial self-assembly of block copolymers on lithographically defined nanopatterned substrates Nature $\mathbf{4 2 4}$ 411-4

[508] Ginzburg V V, Weinhold J D, Hustad P D and Trefonas Iii P 2013 Modeling Chemoepitaxy of Block Copolymer Thin Films using Self-Consistent Field Theory $J$. Photopolym. Sci. Technol. 26 817-23

[509] Edwards E W, Stoykovich M P, Müller M, Solak H H, de Pablo J J and Nealey P F 2005 Mechanism and kinetics of ordering in diblock copolymer thin films on chemically nanopatterned substrates J. Polym. Sci., Part B: Polym. Phys. 43 3444-59

[510] Sivaniah E, Hayashi Y, Iino M, Hashimoto T and Fukunaga K 2003 Observation of Perpendicular Orientation in Symmetric Diblock Copolymer Thin Films on Rough Substrates Macromol. 36 5894-6

[511] Tsori Y, Sivaniah E, Andelman D and Hashimoto T 2005 Orientational Transitions in Symmetric Diblock Copolymers on Rough Surfaces Macromol. 38 7193-6 
[512] Kundu S, Ganesan R, Gaur N, Saifullah M S M, Hussain H, Yang H and Bhatia C S 2012 Effect of angstrom-scale surface roughness on the self-assembly of polystyrenepolydimethylsiloxane block copolymer Sci. Rep. 2

[513] Kim T, Wooh S, Son J G and Char K 2013 Orientation Control of Block Copolymer Thin Films Placed on Ordered Nanoparticle Monolayers Macromol. 46 8144-51

[514] Hong S W, Huh J, Gu X, Lee D H, Jo W H, Park S, Xu T and Russell T P 2012 Unidirectionally aligned line patterns driven by entropic effects on faceted surfaces Proc. Natl. Acad. Sci.

[515] Peng M, Ma S, Hu J and Wang R 2015 Hierarchical nanostructures of diblock copolymer thin films directed by a saw-toothed substrate Soft Matter 11 6642-51

[516] Ho-Cheol K, Charles T R and Linnea S 2008 Fabrication of 20nm half-pitch gratings by corrugation-directed self-assembly Nanotechnology 19235301

[517] Tavakkoli K. G A, Nicaise S M, Gadelrab K R, Alexander-Katz A, Ross C A and Berggren K K 2016 Multilayer block copolymer meshes by orthogonal self-assembly Nat Commun 7

[518] Zhang X, Yager K G, Douglas J F and Karim A 2014 Suppression of target patterns in domain aligned cold-zone annealed block copolymer films with immobilized filmspanning nanoparticles Soft Matter $103656-66$

[519] Segalman R A, Hexemer A and Kramer E J 2003 Effects of Lateral Confinement on Order in Spherical Domain Block Copolymer Thin Films Macromol. 36 6831-9

[520] Sundrani D, Darling S B and Sibener S J 2003 Guiding Polymers to Perfection: Macroscopic Alignment of Nanoscale Domains Nano Lett. 4 273-6

[521] Sundrani D, Darling S B and Sibener S J 2004 Hierarchical Assembly and Compliance of Aligned Nanoscale Polymer Cylinders in Confinement Langmuir 20 5091-9

[522] Hammond M R, Cochran E, Fredrickson G H and Kramer E J 2005 Temperature Dependence of Order, Disorder, and Defects in Laterally Confined Diblock Copolymer Cylinder Monolayers Macromol. 38 6575-85

[523] Ruiz R, Ruiz N, Zhang Y, Sandstrom R L and Black C T 2007 Local Defectivity Control of 2D Self-Assembled Block Copolymer Patterns Adv. Mater. 19 2157-62

[524] Bates C M, Seshimo T, Maher M J, Durand W J, Cushen J D, Dean L M, Blachut G, Ellison C J and Willson C G 2012 Polarity-Switching Top Coats Enable Orientation of Sub-10-nm Block Copolymer Domains Science 338 775-9

[525] Yoshida H, Suh H S, Ramirez-Herunandez A, Lee J I, Aida K, Wan L, Ishida Y, Tada Y, Ruiz R, de Pablo J and Nealey P F 2013 Topcoat Approaches for Directed Self-Assembly of Strongly Segregating Block Copolymer Thin Films J. Photopolym. Sci. Technol. 26 55-8

[526] Kim E, Kim W, Lee K H, Ross C A and Son J G 2014 A Top Coat with Solvent Annealing Enables Perpendicular Orientation of Sub-10 nm Microdomains in SiContaining Block Copolymer Thin Films Advanced Functional Materials 24 6981-8

[527] Maher M J, Bates C M, Blachut G, Sirard S, Self J L, Carlson M C, Dean L M, Cushen J D, Durand W J, Hayes C O, Ellison C J and Willson C G 2014 Interfacial Design for Block Copolymer Thin Films Chem. Mater. 26 1471-9

[528] Jeong J, Ha J S, Lee S-S and Son J G 2015 Topcoat-Assisted Perpendicular and Straightly Parallel Coexisting Orientations of Block Copolymer Films Macromol. Rapid Commun. 36 1261-6 
[529] Seshimo T, Maeda R, Odashima R, Takenaka Y, Kawana D, Ohmori K and Hayakawa T 2016 Perpendicularly oriented sub-10-nm block copolymer lamellae by atmospheric thermal annealing for one minute Scientific Reports 619481

[530] Zhang J, Clark M B, Wu C, Li M, Trefonas P and Hustad P D 2016 Orientation Control in Thin Films of a High- $\chi$ Block Copolymer with a Surface Active Embedded Neutral Layer Nano Lett. 16 728-35

[531] Durand W J, Carlson M C, Maher M J, Blachut G, Santos L J, Tein S, Ganesan V, Ellison C J and Willson C G 2016 Experimental and Modeling Study of Domain Orientation in Confined Block Copolymer Thin Films Macromol. 49 308-16

[532] Amundson K, Helfand E, Quan X and Smith S D 1993 Alignment of lamellar block copolymer microstructure in an electric field. 1. Alignment kinetics Macromol. 26 2698703

[533] Morkved T L, Lu M, Urbas A M, Ehrichs E E, Jaeger H M, Mansky P and Russell T P 1996 Local Control of Microdomain Orientation in Diblock Copolymer Thin Films with Electric Fields Science 273 931-3

[534] Grigorova T, Pispas S, Hadjichristidis N and Thurn-Albrecht T 2005 Magnetic Field Induced Orientation in Diblock Copolymers with One Crystallizable Block Macromol. 38 $7430-3$

[535] Xu T, Zhu Y, Gido S P and Russell T P 2004 Electric Field Alignment of Symmetric Diblock Copolymer Thin Films Macromol. 37 2625-9

[536] Park M J and Balsara N P 2010 Anisotropic Proton Conduction in Aligned Block Copolymer Electrolyte Membranes at Equilibrium with Humid Air Macromol. 43 292-8

[537] Xu T, Zvelindovsky A V, Sevink G J A, Lyakhova K S, Jinnai H and Russell T P 2005 Electric Field Alignment of Asymmetric Diblock Copolymer Thin Films Macromol. 38 10788-98

[538] Olszowka V, Hund M, Kuntermann V, Scherdel S, Tsarkova L and Böker A 2009 Electric Field Alignment of a Block Copolymer Nanopattern: Direct Observation of the Microscopic Mechanism ACS Nano 3 1091-6

[539] Gopinadhan M, Majewski P W, Choo Y and Osuji C O 2013 Order-Disorder Transition and Alignment Dynamics of a Block Copolymer Under High Magnetic Fields by Itextit $\{$ In Situ $\}$ X-Ray Scattering Phys. Rev. Lett. 110078301

[540] Gopinadhan M, Majewski P W, Beach E S and Osuji C O 2012 Magnetic Field Alignment of a Diblock Copolymer Using a Supramolecular Route ACS Macro Letters 1 184-9

[541] Böker A, Elbs H, Hänsel H, Knoll A, Ludwigs S, Zettl H, Urban V, Abetz V, Müller A H E and Krausch G 2002 Microscopic Mechanisms of Electric-Field-Induced Alignment of Block Copolymer Microdomains Phys. Rev. Lett. 89135502

[542] Gopinadhan M, Deshmukh P, Choo Y, Majewski P W, Bakajin O, Elimelech M, Kasi R M and Osuji C O 2014 Thermally Switchable Aligned Nanopores by Magnetic-Field Directed Self-Assembly of Block Copolymers Adv. Mater. 26 5148-54

[543] Liedel C, Pester C W, Ruppel M, Lewin C, Pavan M J, Urban V S, Shenhar R, Bösecke P and Böker A 2013 Block Copolymer Nanocomposites in Electric Fields: Kinetics of Alignment ACS Macro Letters 2 53-8

[544] Morrison F, Le Bourvellec G and Winter H H 1987 Flow-induced structure and rheology of a triblock copolymer J. Appl. Polym. Sci. 33 1585-600 
[545] Winey K I, Patel S S, Larson R G and Watanabe H 1993 Interdependence of shear deformations and block copolymer morphology Macromol. 26 2542-9

[546] Angelescu D E, Waller J H, Register R A and Chaikin P M 2005 Shear-Induced Alignment in Thin Films of Spherical Nanodomains Adv. Mater. 17 1878-81

[547] Davis R L, Chaikin P M and Register R A 2014 Cylinder Orientation and Shear Alignment in Thin Films of Polystyrene-Poly(n-hexyl methacrylate) Diblock Copolymers Macromol. 47 5277-85

[548] Pujari S, Keaton M A, Chaikin P M and Register R A 2012 Alignment of perpendicular lamellae in block copolymer thin films by shearing Soft Matter 8 5358-63

[549] Marencic A P, Adamson D H, Chaikin P M and Register R A 2010 Shear alignment and realignment of sphere-forming and cylinder-forming block-copolymer thin films Phys. Rev. E 81011503

[550] Marencic A P, Chaikin P M and Register R A 2012 Orientational order in cylinderforming block copolymer thin films Phys. Rev. E 86021507

[551] Pelletier V, Asakawa K, Wu M, Adamson D H, Register R A and Chaikin P M 2006 Aluminum nanowire polarizing grids: Fabrication and analysis Appl. Phys. Lett. 88 211114

[552] Borah D, Senthamaraikannan R, Rasappa S, Kosmala B, Holmes J D and Morris M A 2013 Swift Nanopattern Formation of PS-b-PMMA and PS-b-PDMS Block Copolymer Films Using a Microwave Assisted Technique ACS Nano 7 6583-96

[553] Qiang Z, Ye C, Lin K, Becker M L, Cavicchi K A and Vogt B D 2016 Evolution in surface morphology during rapid microwave annealing of PS-b-PMMA thin films $J$. Polym. Sci., Part B: Polym. Phys. n/a-n/a

[554] Borah D, Shaw M T, Holmes J D and Morris M A 2013 Sub-10 nm Feature Size PS-bPDMS Block Copolymer Structures Fabricated by a Microwave-Assisted Solvothermal Process ACS Applied Materials \& Interfaces 5 2004-12

[555] Jin C, Murphy J N, Harris K D and Buriak J M 2014 Deconvoluting the Mechanism of Microwave Annealing of Block Copolymer Thin Films ACS Nano 8 3979-91

[556] Mokarian-Tabari P, Cummins C, Rasappa S, Simao C, Sotomayor Torres C M, Holmes J D and Morris M A 2014 Study of the Kinetics and Mechanism of Rapid Self-Assembly in Block Copolymer Thin Films during Solvo-Microwave Annealing Langmuir 30 10728-39

[557] Pfann W G 1962 Zone Melting: This technique offers unique advantages in purification and in control of composition in various substances Science 135 1101-9

[558] Chung Y S, Shin N, Kang J, Jo Y, Prabhu V M, Satija S K, Kline R J, DeLongchamp D M, Toney M F, Loth M A, Purushothaman B, Anthony J E and Yoon D Y 2010 ZoneRefinement Effect in Small Molecule-Polymer Blend Semiconductors for Organic ThinFilm Transistors J. Am. Chem. Soc. 133 412-5

[559] Lovinger A J, Chua J O and Gryte C C 1977 Studies on the $\alpha$ and $\beta$ forms of isotactic polypropylene by crystallization in a temperature gradient J. Polym. Sci., Part B: Polym. Phys. 15 641-56

[560] Bodycomb J, Funaki Y, Kimishima K and Hashimoto T 1999 Single-Grain Lamellar Microdomain from a Diblock Copolymer Macromol. 32 2075-7

[561] Hashimoto T, Bodycomb J, Funaki Y and Kimishima K 1999 The Effect of Temperature Gradient on the Microdomain Orientation of Diblock Copolymers Undergoing an OrderDisorder Transition Macromol. 32 952-4 
[562] Mita K, Tanaka H, Saijo K, Takenaka M and Hashimoto T 2007 Cylindrical Domains of Block Copolymers Developed via Ordering under Moving Temperature Gradient Macromol. 40 5923-33

[563] Mita K, Tanaka H, Saijo K, Takenaka M and Hashimoto T 2008 Macroscopically oriented lamellar microdomains created by "cold zone-heating" method involving OOT Polymer 49 5146-57

[564] Mita K, Takenaka M, Hasegawa H and Hashimoto T 2008 Cylindrical Domains of Block Copolymers Developed via Ordering under Moving Temperature Gradient: Real-Space Analysis Macromol. 41 8789-99

[565] Mita K, Tanaka H, Saijo K, Takenaka M and Hashimoto T 2008 Ordering of Cylindrical Domains of Block Copolymers under Moving Temperature Gradient: Separation of TInduced Ordering from Surface-Induced Ordering Macromol. 41 6787-92

[566] Mita K, Tanaka H, Saijo K, Takenaka M and Hashimoto T 2008 Ordering of Cylindrical Domain of Block Copolymers under Moving Temperature Gradient: Effects of Moving Rate Macromol. 41 6780-6

[567] De Rosa C, Park C, Thomas E L and Lotz B 2000 Microdomain patterns from directional eutectic solidification and epitaxy Nature $\mathbf{4 0 5} 433$

[568] Park C, Cheng J Y, Fasolka M J, Mayes A M, Ross C A, Thomas E L and De Rosa C 2001 Double textured cylindrical block copolymer domains via directional solidification on a topographically patterned substrate Appl. Phys. Lett. 79 848-50

[569] Angelescu D E, Waller J H, Adamson D H, Register R A and Chaikin P M 2007 Enhanced Order of Block Copolymer Cylinders in Single-Layer Films Using a Sweeping Solidification Front Adv. Mater. 19 2687-90

[570] Bosse A W, Douglas J F, Berry B C, Jones R L and Karim A 2007 Block-Copolymer Ordering with a Spatiotemporally Heterogeneous Mobility Phys. Rev. Lett. 99 216101-4

[571] Zhang H, Zhang J, Yang Y and Zhou X 1997 Microphase separation of diblock copolymer induced by directional quenching The Journal of Chemical Physics 106 78492

[572] Cong Z, Zhang L, Wang L and Lin J 2016 Understanding the ordering mechanisms of self-assembled nanostructures of block copolymers during zone annealing The Journal of Chemical Physics 144114901

[573] Singh G, Yager K G, Smilgies D-M, Kulkarni M M, Bucknall D G and Karim A 2012 Tuning Molecular Relaxation for Vertical Orientation in Cylindrical Block Copolymer Films via Sharp Dynamic Zone Annealing Macromol. 45 7107-17

[574] Berry B C, Singh G, Kim H-C and Karim A 2013 Highly Aligned Block Copolymer Thin Films by Synergistic Coupling of Static Graphoepitaxy and Dynamic Thermal Annealing Fields ACS Macro Letters 2 346-50

[575] Rao T, Singh G, Xie S, Karim A and Becker M L 2013 2-D gold nanoparticle arrays from thermally directed self-assembly of peptide-derivatized block copolymers Soft Matter 9 8023-32

[576] Samant S, Hailu S T, Al-Enizi A M, Karim A and Raghavan D 2015 Orientation control in nanoparticle filled block copolymer cold zone annealed films J. Polym. Sci., Part B: Polym. Phys. 53 604-14

[577] Xue J, Singh G, Qiang Z, Karim A and Vogt B D 2013 Unidirectional self-assembly of soft templated mesoporous carbons by zone annealing Nanoscale 5 7928-35 
[578] Ye C, Sun Y, Karim A and Vogt B D 2015 Extending Dynamic Range of Block Copolymer Ordering with Rotational Cold Zone Annealing (RCZA) and Ionic Liquids Macromol. 48 7567-73

[579] Singh G, Batra S, Zhang R, Yuan H, Yager K G, Cakmak M, Berry B and Karim A 2013 Large-Scale Roll-to-Roll Fabrication of Vertically Oriented Block Copolymer Thin Films ACS Nano 7 5291-9

[580] Cakmak M, Batra S and Yalcin B 2015 Field assisted self-assembly for preferential through thickness ("z-direction") alignment of particles and phases by electric, magnetic, and thermal fields using a novel roll-to-roll processing line Polymer Engineering \& Science 55 34-46

[581] Jacobs A G, Jung B, Ober C K and Thompson M O 2014 Control of PS-b-PMMA directed self-assembly registration by laser induced millisecond thermal annealing. pp 90492B-B-7

[582] Jin H M, Lee S H, Kim J Y, Son S-W, Kim B H, Lee H K, Mun J H, Cha S K, Kim J S, Nealey P F, Lee K J and Kim S O 2016 Laser Writing Block Copolymer Self-Assembly on Graphene Light-Absorbing Layer ACS Nano 10 3435-42

[583] Ye C, Singh G, Wadley M L, Karim A, Cavicchi K A and Vogt B D 2013 Anisotropic Mechanical Properties of Aligned Polystyrene-block-polydimethylsiloxane Thin Films Macromol. 46 8608-15

[584] Zhang R, Singh G, Dang A, Dai L, Bockstaller M R, Akgun B, Satija S and Karim A 2013 Nanoparticle-Driven Orientation Transition and Soft-Shear Alignment in Diblock Copolymer Films via Dynamic Thermal Gradient Field Macromol. Rapid Commun. 34 1642-7

[585] Majewski P W and Yager K G 2015 Block Copolymer Response to Photothermal Stress Fields Macromol. 48 4591-8

[586] Davis R L, Michal B T, Chaikin P M and Register R A 2015 Progression of Alignment in Thin Films of Cylinder-Forming Block Copolymers upon Shearing Macromol. 48 533947

[587] Nikoubashman A, Davis R L, Michal B T, Chaikin P M, Register R A and Panagiotopoulos A Z 2014 Thin Films of Homopolymers and Cylinder-Forming Diblock Copolymers under Shear ACS Nano 8 8015-26

[588] Wan L, Ji S, Liu C-C, Craig G S W and Nealey P F 2016 Directed self-assembly of solvent-vapor-induced non-bulk block copolymer morphologies on nanopatterned substrates Soft Matter 12 2914-22

[589] Qiang Z, Zhang Y, Wang Y, Bhaway S M, Cavicchi K A and Vogt B D 2015 Highly aligned, large pore ordered mesoporous carbon films by solvent vapor annealing with soft shear Carbon 82 51-9

[590] Kathrein C C, Bai W, Currivan-Incorvia J A, Liontos G, Ntetsikas K, Avgeropoulos A, Böker A, Tsarkova L and Ross C A 2015 Combining Graphoepitaxy and Electric Fields toward Uniaxial Alignment of Solvent-Annealed Polystyrene-b-Poly(dimethylsiloxane) Block Copolymers Chem. Mater. 27 6890-8

[591] Sinturel C, Bates F S and Hillmyer M A 2015 High $\chi$-Low N Block Polymers: How Far Can We Go? ACS Macro Letters 1044-50

[592] Luo Y, Montarnal D, Kim S, Shi W, Barteau K P, Pester C W, Hustad P D, Christianson M D, Fredrickson G H, Kramer E J and Hawker C J 2015 Poly(dimethylsiloxane-b- 
methyl methacrylate): A Promising Candidate for Sub-10 nm Patterning Macromol. 48 3422-30

[593] Deng H, Xie N, Li W, Qiu F and Shi A-C 2015 Perfectly Ordered Patterns via CornerInduced Heterogeneous Nucleation of Self-Assembling Block Copolymers Confined in Hexagonal Potential Wells Macromol. 48 4174-82

[594] Yokoyama H 2006 Diffusion of block copolymers Mater. Sci. Eng., $R 53$ 199-248

[595] Green P F 1995 Translational Dynamics of Diblock Copolymers Macromol. 28 2155-8

[596] Eastman C E and Lodge T P 1994 Self-diffusion and tracer diffusion in styrene/2vinylpyridine block copolymer melts Macromol. 27 5591-8

[597] Lodge T P, Hamersky M W, Milhaupt J M, Kannan R M, Dalvi M C and Eastman C E 1997 Diffusion in microstructured block copolymer melts Macromol. Symp. 121 219-33

[598] Baba J, Kubo T, Takano A and Nose T 1994 Molecular weight dependence of the tracer diffusion coefficient of short chains in the microphase domain of block copolymers as studied by the pulsed-field gradient nuclear magnetic resonance method Polymer 35 14550

[599] Santangelo P G and Roland C M 1998 Molecular Weight Dependence of Fragility in Polystyrene Macromol. 31 4581-5

[600] Bergman R, Alvarez F, Alegría A and Colmenero J 1998 Dielectric relaxation in PMMA revisited J. Non-Cryst. Solids 235-237 580-3

[601] Masser K A and Runt J 2010 Dynamics of Polymer Blends of a Strongly Interassociating Homopolymer with Poly(vinyl methyl ether) and Poly(2-vinylpyridine) Macromol. 43 6414-21

[602] Fragiadakis D, Dou S, Colby R H and Runt J 2009 Molecular mobility and Li+ conduction in polyester copolymer ionomers based on poly(ethylene oxide) The Journal of Chemical Physics 130064907

[603] Schoenhals A 1993 Relation between main and normal mode relaxations for polyisoprene studied by dielectric spectroscopy Macromol. 26 1309-12

[604] Ngai K L and Roland C M 1995 Component Dynamics in Polyisoprene/Poly(vinylethylene) Blends Macromol. 28 4033-5

[605] Lorthioir C, Alegría A, Colmenero J and Deloche B 2004 Heterogeneity of the Segmental Dynamics of Poly(dimethylsiloxane) in a Diblock Lamellar Mesophase: Dielectric Relaxation Investigations Macromol. 37 7808-17

[606] Roland C M 2008 Characteristic relaxation times and their invariance to thermodynamic conditions Soft Matter 4 2316-22

[607] Rault J 2000 Origin of the Vogel-Fulcher-Tammann law in glass-forming materials: the $\alpha-\beta$ bifurcation J. Non-Cryst. Solids 271 177-217

[608] Frick B and Richter D 1995 The Microscopic Basis of the Glass Transition in Polymers from Neutron Scattering Studies Science 267 1939-45

[609] Metatla N and Soldera A 2007 The Vogel-Fulcher-Tamman Equation Investigated by Atomistic Simulation with Regard to the Adam-Gibbs Model Macromol. 40 9680-5 